\title{
HEPATOLOGY
}

\section{Trial Design and Endpoints in hepatocellular carcinoma: AASLD Consensus Conference}

\begin{tabular}{|c|c|}
\hline Journal: & Hepatology \\
\hline Manuscript ID & HEP-20-0226.R1 \\
\hline Wiley - Manuscript type: & Original \\
\hline $\begin{array}{r}\text { Date Submitted by the } \\
\text { Author: }\end{array}$ & 31-Mar-2020 \\
\hline Complete List of Authors: & $\begin{array}{l}\text { Llovet, Josep M.; Icahn School of Medicine at Mount Sinai, Division of } \\
\text { Liver Diseases; IDIBAPS, Hospital Clínic, Barcelona. University of } \\
\text { Barcelona, Head, Translational Research in Liver Cancer, Liver Unit; } \\
\text { Institució Catalana d'Estudis Avançats (ICREA) } \\
\text { Villanueva, Augusto; Tisch Cancer Institute, Department of Medicine, } \\
\text { Icahn School of Medicine at Mount Sinai,Liver Cancer Research Program, } \\
\text { Division of Liver Diseases } \\
\text { Marrero, Jorge; UT Southwestern Medical Center, Department of Internal } \\
\text { Medicine } \\
\text { Schwartz, Myron; Icahn School of Medicine at Mount Sinai Tisch Cancer } \\
\text { Institute, } \\
\text { Meyer, Tim; University College London Cancer Institute, Hem/Oncology } \\
\text { Galle, Peter; Mainz University, Department of Internal Medicine I } \\
\text { Lencioni, Riccardo; University of Pisa School of Medicine and Surgery, } \\
\text { Radiology; Miami Cancer Institute } \\
\text { Greten, Tim; Gastrointestinal Malignancy Section, Thoracic and } \\
\text { Gastrointestinal Oncology Branch, Center for Cancer Research, National } \\
\text { Cancer Institute, National Institutes of Health, } \\
\text { Kudo, Masatoshi; Kindai University, Department of Gastroenterology and } \\
\text { Hepatology } \\
\text { Mandrekar, Sumithra J; Mayo Clinic College of Medicine and Science, } \\
\text { Health Sciences Research } \\
\text { Zhu, Andrew; Massachusetts General Hospital, Harvard Medical School, } \\
\text { Oncology; Jiahui International Cancer Center } \\
\text { Finn, Richard; Geffen School of Medicine at UCLA, Medicine- } \\
\text { Hematology/ Oncology } \\
\text { Roberts, Lewis; Mayo Clinic, Gastroenterology \& Hepatology }\end{array}$ \\
\hline Keywords: & $\begin{array}{l}\text { Trial Design, End Points, Randomized control trials, PFS (Progression free } \\
\text { survival), Hepatocellular Carcinoma }\end{array}$ \\
\hline
\end{tabular}

\section{SCHOLARONE" Manuscripts}




\title{
Title: Trial Design and Endpoints in hepatocellular carcinoma: AASLD Consensus Conference.
}

Authors: Josep M. Llovet ${ }^{1,2,3}$ Augusto Villanueva1, Jorge A. Marrero4, Myron Schwartz¹, Tim Meyer ${ }^{5}$, Peter R. Galle ${ }^{6}$, Riccardo Lencioni ${ }^{7,8}$, Tim F. Greten ${ }^{9}$, Masatoshi Kudo ${ }^{10}$, Sumithra J. Mandrekar ${ }^{11}$, Andrew X. Zhu ${ }^{12,13}$, Richard S. Finn ${ }^{14}$, Lewis R. Roberts ${ }^{15}$, for AASLD Panel of experts on Trial Design in HCC.

\begin{abstract}
Affiliations: ${ }^{1}$ Mount Sinai Liver Cancer Program, Division of Liver Diseases, Tisch Cancer Institute, Icahn School of Medicine at Mount Sinai, New York, NY, USA; ${ }^{2}$ Translational Research in Hepatic Oncology, Liver Unit, IDIBAPS, Hospital Clinic, University of Barcelona, Catalonia, Spain; ${ }^{3}$ Institució Catalana d'Estudis Avançats (ICREA), Barcelona, Catalonia, Spain. ${ }^{4} U T$ Southwestern Medical Center, Dallas, Texas; ${ }^{5}$ Dept Oncology, University College London Cancer Institute; ${ }^{6}$. Dept. of Internal Medicine, Mainz University Medical Center, Mainz, Germany; ' Department of Radiology, University of Pisa School of Medicine; 8 Miami Cancer Institute, Miami, FL; ${ }^{9}$ Gastrointestinal Malignancy Section, Thoracic and Gastrointestinal Oncology Branch, Center for Cancer Research, National Cancer Institute, National Institutes of Health, Bethesda, Maryland; ${ }^{10}$ Department of Gastroenterology and Hepatology, Kindai University Faculty of Medicine, Osaka-Sayama, Osaka, Japan. ${ }^{11}$ Department of Health Sciences Research, Mayo Clinic, Rochester, MN. ${ }^{12}$ Massachusetts General Hospital Cancer Center, Harvard Medical School, Boston, MA, USA; ${ }^{13}$ Jiahui International Cancer Center, Shanghai, China; ${ }^{14}$ Geffen School of Medicine at UCLA, California; ${ }^{15}$ Gastroenterology \& Hepatology Dept. Mayo Clinic, Rochester, MN.
\end{abstract}

Conflict of interest: Josep M Llovet is receiving research support from Bayer HealthCare Pharmaceuticals, Eisai Inc, Bristol-Myers Squibb, Ipsen and Boehringer-Ingelheim and consulting fees from Eisai Inc, Merck, Bayer HealthCare Pharmaceuticals, Bristol-Myers Squibb, Celsion Corporation, Eli Lilly, Ipsen, Glycotest, Roche, AstraZeneca, Sirtex, Nucleix and Can-Fite. Augusto Villanueva has received consulting fees from Guidepoint and Fujifilm; advisory board fees from Exact Sciences, Nucleix, Gilead and NGM Pharmaceuticals, and research support from Eisai Pharmaceuticals. Jorge Marrero reports consulting from Glycotest. Peter Galle is receiving research support from Bayer HealthCare Pharmaceuticals and consulting fees from Bayer HealthCare Pharmaceuticals, BristolMyers Squibb, Eisai Inc, Merck, Eli Lilly, Ipsen, Roche, AstraZeneca, Sirtex. Tim Meyer has received advisory board fees from Eisai, Roche, AstraZeneca, Ipsen, BTG and Tarveda. Masatoshi Kudo is receiving lecture fee from Eisai Inc, Bayer, MSD, research support from Eisai Inc, Gilead, Takeda, Otsuka, Taiho, Bristol-Myers Squibb, Ono Pharmaceutical and consulting fees from Bayer, Eisai, Ono, MSD, BMS, Eli Lilly, Roche, AstraZeneca, Chugai. Andrew Zhu reports research funding from Bayer, Bristol-Myers Squibb, Eli Lilly, Merck, and Novartis, and is a consultant/advisory board member for AstraZeneca, Bayer, Bristol-Myers Squibb, Eisai, Eli Lilly, Exelixis, Merck, Novartis, Roche, and Sanofi. Richard Finn reports consulting fees from AstraZeneca, Bayer, BristolMyersSquibb, CStone, Eisai, Eli Lilly, 
Merck, Novartis, Pfizer, Roche/Genentech. Lewis Roberts reports research funding from Bayer, BTG International, Exact Sciences, Gilead Sciences, Glycotest, Redhill Biopharma, TARGET PharmaSolutions, and Wako Diagnostics, and is a consultant/advisory board member for Bayer, Exact Sciences, Gilead Sciences, GRAIL, QED Therapeutics, and TAVEC.

All other co-authors do not have conflicts to disclose.

Acknowledgements: Josep M Llovet is supported by European Commission (EC)/Horizon 2020 Program (HEPCAR, Ref. 667273-2), EIT Health (CRISH2, Ref. 18053), Accelerator Award (CRUCK, AEEC, AIRC) (HUNTER, Ref. C9380/A26813), National Cancer Institute (P30-CA196521), U.S. Department of Defense (CA150272P3), Samuel Waxman Cancer Research Foundation, Spanish National Health Institute (SAF2016-76390) and the Generalitat de Catalunya/AGAUR (SGR-1358). Augusto Villanueva is supported by the Department of Defense (CA150272P3). Tim Greten is supported by the Intramural Research Program of the $\mathrm{NIH}$, National Cancer Institute, Center for Cancer Research. Sumithra J. Mandrekar is supported by a P30CA15083 grant. Andrew Zhu has received research support from V Foundation for Cancer Research, and DOD of Defense Team Science Translational Award. Richard Finn is supported by US Dept of Defense IDEA Award. Tim Meyer is supported by the NIHR UCLH Biomedical Research Centre. Lewis Roberts is supported by the National Cancer Institute (P50 CA210964).

Correspondence: Josep M. Llovet, MD, PhD, FAASLD. Mount Sinai Liver Cancer Program, Division of Liver Diseases, Tisch Cancer Institute, Icahn School of Medicine at Mount Sinai, Madison Ave 1425. 11F-70. Box 1123, New York, NY10029. USA. E-mail: josep.llovet@mssm.edu 


\begin{abstract}
Proper trial design is critical for the success of clinical investigations. Hepatocellular carcinoma (HCC) is a complex disease that has several unique properties. In 2008, after the approval of sorafenib, a panel of experts proposed guidelines for trial design and endpoints in HCC that have been instrumental during the last decade and provided a framework to allow an homogeneous analysis of reported investigations. Since then, several phase III studies have been reported and novel challenges have emerged. A panel of experts conveyed by AASLD organized a Special Topic Conference on trial design and endpoints to address those emerging challenges. This review summarizes the analysis and conclusions of those discussions and provides novel recommendations on the selection endpoints, stratification variables and targeted populations in the complex arena of HCC. We have covered the full spectrum of the disease, from surveillance/ chemoprevention, to neoadjuvant and adjuvant trials after curative therapies, and trials in intermediate and advanced stages of HCC. We explore the prospects for incorporating biomarkers and liquid biopsy into conventional clinical trials. In addition, we address the need for obtaining tissue and blood samples in all investigations and propose novel primary endpoints such as progression free survival with restrictive rules and patient reported outcomes. This up-dated set of recommendations is timely considering the advent of more potent combination therapies in all areas of HCC management, the increase in adverse events associated with those combinations, and the evidence that several lines of effective treatments will benefit a given patient. We herein articulate a framework to facilitate capturing the efficacy of novel therapeutic strategies with the goal of improving the outcomes of patients suffering from this disease.
\end{abstract}




\section{Introduction}

Hepatocellular carcinoma (HCC) is the fourth leading cause of cancer-related mortality worldwide $(1,2)$. This neoplasm has some unique characteristics. It occurs in most cases complicating underlying cirrhosis, has specific non-invasive criteria for diagnosis, follows a unique staging system and historically has been resistant to conventional chemotherapy. Several treatments have achieved adoption as standard of care according to clinical practice guidelines, including potentially curative therapies (i.e. resection, liver transplantation and local ablation) for early tumors, transarterial chemoembolization (TACE) for intermediate stage tumors, and systemic drugs for advanced tumors in front line (sorafenib and lenvatinib) and second line (regorafenib, cabozantinib and ramucirumab)(3$5)$. Life expectancy has improved progressively in all stages of the disease. Effective implementation of surveillance for patients at risk of developing HCC and access to current proven therapies has been a milestone. Overall, median survival times beyond 5 years are expected for early stages, about 20-30 months for intermediate stages, and between 10-16 months for advanced stage HCC [Fig. 1(3,4,6)]. Novel drugs and combinations continue to enter the research arena to address unmet medical needs. All these research activities require precise endpoints and tools for measuring clinical benefits.

Thus, clinical trial design has become a major focus of attention in HCC research. Since randomized controlled trials are the main source of evidence for drug approvals in oncology, it is of paramount relevance to understand the critical endpoints and tools for measuring them, as well as optimal strategies for identifying and enrolling target populations and for patient stratification. It has become evident that a deep understanding of factors determining HCC outcomes and trial design is required to achieve optimal results. There are trials with a non-inferiority design that have been positive, and lead to drug approval, while others demonstrate superior outcomes in surrogate end-points, such as progression free survival (PFS) or patient reported outcomes but not in the primary endpoint of overall survival. Some recent trials have also been reported to be statistically negative but "clinically positive". It is important to understand the reasons for the failure or success of a trial in order to move the field forward. In addition, while several positive phase III trials for advanced HCC have recently changed clinical practice (7-11), no major advances have occurred in the area of surveillance and early detection, adjuvant therapies after resection/ablation or management of intermediate stage HCC over the last 15 years. The lack of effective novel drugs/devices may be the cause of negative studies in these areas, but suboptimal trial design may also have jeopardized the likelihood of a positive result. With this challenge in mind, the Hepatobiliary Neoplasia Special Interest Group of the American Association for the Study of Liver Disease (AASLD) organized a Single Topic Conference in Atlanta in 2019 to address these issues. This position paper summarizes the major concepts discussed in the conference with the aim of updating the proposals previously reported by a similar AASLD panel in 2008(12).

\section{Overview on trial design and endpoints}

Clinical trials are essential to establish the clinical efficacy of new therapeutic interventions. They are instrumental in developing clinical practice guidelines and form the basis for evidence-based medicine(13). An adequate clinical trial design is crucial, as an effective drug can be discarded due to a poor trial design and vice-versa. The main considerations when designing a clinical trial are to: a) select a well-defined target patient population (i.e., inclusion and exclusion criteria), b) pre-specify clear endpoints (primary and secondary) and data analysis plan, c) specify randomization and allocation method; and d) secure efficacy of randomization (stratification at enrollment for prognostic variables). Based on these and other variables, the quality of clinical trials can be quantified using different scores such as 
the Jadad score(14), the Delphi List(15), the CONSORT statement(16,17), and the Cochrane Back Review Group criteria(18). Until the SHARP trial(11), which established the benefit of sorafenib in advanced stages HCC patients, the quality of the trials conducted in HCC was commonly modest. A systematic review found that only $50 \%$ of the clinical trials reported between 2002 and 2005 in HCC were deemed high quality as per the modified Jadad score(19). The 2008 position paper resulting from the AASLD conference provided a useful framework for academic centers, industry partners and regulators on the design of trials in $\mathrm{HCC}(12)$. Subsequently, the quality of clinical trials assessing systemic therapies has significantly improved. There has been less activity in terms of high-end clinical trials in other treatment areas. This position paper will extensively discuss the singularities of trial design in every clinical aspect of HCC management.

In clinical trials, the benefit of an intervention is quantified using endpoints, which are predefined events that once reached exclude the patient from further evaluation within the trial. There are 3 main types of endpoints: hard, surrogate and patient-reported, all extensively described elsewhere(20). Hard endpoints are well defined and easy to measure objectively. The archetypes of a hard endpoint are overall survival (OS) or cancer-related survival. Surrogate endpoints, such as progression-free survival or time to progression, partially rely on the quantification of tumor response, generally using imaging techniques and pre-specified criteria(21). Surrogate endpoints are more vulnerable than hard endpoints, but they have several advantages including their convenience in terms of event accumulation and trial feasibility. Patient-reported endpoints, sometimes referred as soft endpoints, are subjective measures such as quality of life (QoL), in most instances obtained from questionnaires. Overall recommendations of trial design and endpoints in $\mathrm{HCC}$ are detailed in Table 1, whereas expected outcomes for standard of care therapeutic interventions within these trials are summarized in Table 2.

\section{Endpoints}

OS is defined by the time between patient randomization and death from any cause. OS is usually recommended as the primary endpoint for randomized phase III clinical trials(12). OS is the endpoint most frequently used by regulatory agencies to approve drugs as it is objective and clinically relevant. In HCC, as most patients suffer from concomitant cirrhosis, death can result from competing risks, mainly liver toxicity and failure. This fact underscores the need for detailed assessments of safety with any intervention in this population. It is important to capture adverse events in early phase clinical studies as well as in larger randomized studies. Failures in phase 3 studies have been seen from new agents that are more toxic in an HCC population than in other tumor types(22). The competing risk of cirrhosis can introduce bias when evaluating the anti-tumoral activity of a therapeutic intervention, but it can be easily controlled by imposing stringent inclusion criteria in terms of liver function (i.e., Child-Pugh score A without hepatic decompensation). OS has some limitations such as the long follow-up time required to capture the number of events needed to verify significantly improved survival in the experimental arm(23). This can be a critical limitation when exploring interventions at early or intermediate stages. Also, OS can be confounded by sequential therapies received by patients after tumor progression, which for instance affected $30 \%$ of patients enrolled in the lenvatinib trial(7) and up to $50 \%$ of patients in the Checkmate 459 comparing nivolumab vs sorafenib(24). Hence, there is a need to develop surrogate endpoints, which are defined as outcomes not inherently meaningful from the clinical standpoint, but thought to accurately predict hard outcomes such as OS(25).

The main surrogate endpoints in oncology are progression-free survival (PFS), time-toprogression (TTP) and objective response rate (ORR). PFS is the time between patient 
randomization and death or radiological tumor progression, whichever occurs first. There are different tools assessing tumor response with imaging. The most established tool for measuring tumor response in oncology are the RECIST criteria(26), initially developed to evaluate response to cytotoxic drugs. These criteria were adapted to account for HCC singularities in the modified RECIST (mRECIST) version, which incorporates viable tumour detected with arterial enhancement as a key component to evaluate response $(21,27)$. Using mRECIST criteria increases the percentage of subjects who achieve objective response compared to standard RECIST, as shown in different studies of systemic therapies (28-32). A recent meta-analysis evaluated the power of PFS to predict OS in phase 3 trials testing systemic therapies in advanced $\mathrm{HCC}(20)$. The study found a moderate correlation between PFS and OS in 21 RCTs. The authors proposed a conservative surrogate threshold of $\leq 0.6$ for hazard ratio of PFS to predict clinically relevant improvements in OS(33). TTP is defined as the time elapsed between patient randomization and radiological tumor progression. Scheduling repeated radiological assessment of response every 6-8 weeks is mandatory for patients included in trials. Data from SHARP and subsequent studies challenges the implied correlation between TTP and OS. The type of progression may also have clinical implications (34). Survival is worse if patients develop a new extrahepatic lesion and/or vascular invasion as opposed to tumor progression resulting from growth of an existing lesion or a new intrahepatic lesion. Lastly, ORR is the percentage of patients with an objective tumor response, and its correlation with OS is worse than for PFS or TTP(20). This is partially inherent to the use of odds ratios instead of hazard ratios for ORR and also to the fact that only a small proportion of patients achieve an objective response $(<25 \%$, for approved drugs in advanced HCC), which is the event that correlates with OS(31). Nonetheless, ORR has been reported as an independent predictor of survival in early HCC treated with radiofrequency ablation, intermediate treated with TACE and advanced HCC treated with $\mathrm{TKI}(27)$. The impact of the duration of response, which has been reported to be around 12 months for checkpoint inhibitors vs. less than 6 months for TKIs, has not yet been properly incorporated into response assessment. The same is true to small reductions in tumor size not reaching standard thresholds for objective response. In some cases, duration of disease control may be more clinically relevant than the extent of reduction in tumor size. Also, in the case of immune checkpoint inhibitors, tumor response can have a longer lagtime compared to other molecular therapies and can even mimic progression shortly after treatment initiation (i.e., pseudo-progression(35)). This has led to the development of immune-related response criteria(36), which require confirmation of progression at least 4 weeks after progressive disease is first documented.

Surrogate endpoints are frequently used by the Food and Drug Administration (FDA) to approve drugs under the accelerated program, which was initially developed to facilitate early access to new antivirals during the worst years of the HIV epidemic(37). In HCC, the FDA has used ORR and duration of response to grant accelerated approval of the immune checkpoint inhibitors (CPI) nivolumab(38), pembrolizumab(39) and recently the combination of ipilimumab and nivolumab(40). Accelerated approval is not universal and includes some subjectivity from regulators in regards to the strength of the evidence to support approval without a randomized phase 3 study $(24,41)$. In addition, while the use of ORR and other surrogate endpoints may be used to support regulatory approval, they do not necessarily support inclusion in guidelines which often adhere to a higher level of evidence. Despite their common use, surrogate endpoints are vulnerable to interpretation bias. Besides the strength of the endpoint, it is key to determine when the benefit provided by a new therapy is really clinically meaningful. This can be controversial, depending on factors such as the perceptions of patients, providers, health insurers and regulators. In HCC, there is no set threshold that defines a clinically meaningful benefit, but some authors have suggested a hazard ratio cutoff of OS $\leq 0.8$ as a starting point for clinical trial design(42). In fact, all 
positive trials in HCC have led to significant differences in survival with HR below this threshold.

\section{Surveillance for Hepatocellular Carcinoma: design and endpoints}

Surveillance for HCC is one of the milestones advancing the management of HCC, despite that there is not unquestionable data directly supporting a decrease in cancer-related death in persons on surveillance(43). Ultrasound (US), with or without alpha-fetoprotein (AFP), performed every six months is the current standard and is recommended for surveillance of patients with cirrhosis of any cause or chronic hepatitis B without cirrhosis above a regional and gender appropriate age cut-off determined by expert liver societies $(3,4)$. Overall, the implementation of those programs to all targeted populations is modest, and current data report detection of HCC in the setting of surveillance in between $30-50 \%$ of cases(44). In meta-analysis, the pooled sensitivity and specificity of US alone has been shown to be $53 \%$ (95\% Cl: 35-70) and 91\% (95\% Cl:86-94), respectively, while the combination of US and AFP has a sensitivity of $63 \%(95 \% \mathrm{Cl}: 48-75)$ and a specificity of $85 \%(95 \% \mathrm{Cl}: 77-89)(45)$. Due to the relatively low sensitivity and specificity of this approach for detecting early stage HCC, particularly in North America, where high rates of central obesity decrease the performance of ultrasound, a recent study showed that this strategy leads to $27 \%$ of patients with cirrhosis experiencing harms such as follow up testing (CT, MRI, liver biopsy) performed for false-positive or indeterminate results(46). Further, due to low implementation of comprehensive strategies for HCC surveillance, more than $60 \%$ of HCCs in North America, Europe, Africa and large parts of Asia, excepting Taiwan and Japan, are diagnosed with intermediate or advanced stage $\mathrm{HCC}(47)$. There is, therefore, an urgent need for better performing, low cost surveillance strategies in $\mathrm{HCC}$, and accounting for both the benefits and harms of surveillance strategies is important.

Within this overriding context, there is excitement time because advances in genetic, epigenetic, proteomic, glycoproteomic and metabolomic analyses in have enabled large scale multi-omic analyses of HCC tissues, circulating tumor DNA, plasma and serum, resulting in the accelerated identification of novel biomarkers(48-51). Models using standard biostatistical and machine learning and artificial intelligence (AI) approaches are using biomarkers combined with clinical parameters to identify persons at highest risk for HCC. Models and biomarkers under active exploration include the GALAD (Gender, Age, AFP-L3, AFP, and Des-carboxy-prothrombin) score(52), novel glycoproteins (fucosylated kininogen)(53), liquid biopsy analyses of circulating tumor DNA for differentially methylated regions(54)(55), and imaging with abbreviated $\mathrm{MRI}(56)$. Creating the framework for validation of future surveillance is critically important.

To guide the development and evaluation of new surveillance strategies for clinical utilization, a 5-phase program has been developed by the $\mathrm{NCl}$ - Early Detection Research Network (EDRN) for biomarker that uses human samples (blood or human tissue) as well as imaging tests (57). Table 3 shows recommended phases of surveillance test validation, including trial design for studies for HCC surveillance. Phase 1 are biomarker discovery or exploratory studies. Phase 2 studies estimate the ability of a test to distinguish early stage $\mathrm{HCC}$ from those with cirrhosis without HCC. It is important to test for confounders such as age, etiology of liver disease, and liver function; and to have adequate sample size and power. Phase 3 studies enroll at risk individuals and follow them for clinical diagnosis of HCC using prospective-specimen collection, retrospective-blinded evaluation (PRoBE) design (58). The aim is to evaluate, as a function of time before clinical diagnosis, the capacity of the test to detect preclinical HCC; and also, to define the criteria for a positive surveillance test in preparation for phase 4 and 5 studies. Thus, Phases 1-3 rely on retrospective analysis of stored data and specimens. Phase 4 studies require the new test 
be applied to patients with cirrhosis in the clinical setting to assess test performance in HCC detection and false positive and negative rates. Depending on the test under study it may be possible to skip Phase 4 if the test is already used for patient care, for example, evaluating an MRI for surveillance of HCC. Phase 5 studies are randomized trials comparing the new surveillance tests against the standard of care, in the case of HCC the standard should be US with or without AFP, with the aim of determining whether the test can reduce mortality at the population level.

When performing surveillance studies in patients with cirrhosis it is important to enrich the at risk population in order to achieve a sufficient number of incident HCCs in a reasonable time period. Enriching cohorts with patients of older age, viral hepatitis, male sex, Hispanic ethnicity, history of diabetes, and family history of HCC should be considered $(59,60)$. Alternatively, known independent factors associated with HCC development are abnormal bilirubin and platelet count $<100,000 / \mathrm{mm}^{3}$. There is also a need to study currently important populations such as those with non-alcoholic fatty liver disease-related cirrhosis, those with hepatitis C-related cirrhosis who have achieved a sustained virological response after antiviral treatment, and those with suppressed hepatitis B infection on antiviral treatment. These three specific populations will be the most important etiological risk factors in the next decade and their HCC incidence rates (around 1\%/year) appear lower than in previous at risk populations(61). Methods for risk stratification within these populations will therefore become increasingly important for improving the effectiveness of surveillance strategies and programs. Models such as the REAL-B and PAGE-B scores, incorporating male sex, age, alcohol use, baseline cirrhosis, diabetes, platelet count and AFP, allow improved risk stratification of patients on oral antiviral therapy for chronic hepatitis B and could potentially be incorporated into surveillance programs(62).

An important potential confounder in studies that compare the performance of novel biomarkers to current surveillance strategies is the incorporation of imaging by ultrasound or other radiologic modalities into the standard of care. This may confound the results if ultrasound is also used as part of the control arm for the study, as ultrasonography is itself typically part of the gold standard process for determining whether a patient has HCC. Thus, patients with HCCs that are not visible by ultrasound may be falsely determined to be negative for cancer and a positive biomarker test erroneously labeled as a false positive. It is therefore important to use a different high-accuracy imaging modality such as multiphasic $\mathrm{MRI}$ as a gold standard in studies for which ultrasonography is part of the surveillance strategy. However, use of MRI may add substantial cost to the study and may also result in visualization of a number of small indeterminate false positive lesions that are seen on MRI and require follow up investigation, a component of the harms associated with surveillance. While studies of the performance of ultrasound with or without AFP in the clinical care setting have shown suboptimal performance in detection of HCC in at risk individuals, it is not clear what the performance characteristics are for phase 2, 3 or 4 biomarker studies that would meet the threshold for FDA approval as a surveillance test. In general, the FDA guidelines for supporting biomarker qualification recommend that analyses intended to support biomarker qualification should be specified in an analysis plan with a prospectiveretrospective design before analyzing the data. The FDA provides no set quantitative criteria for determining the relationship between the biomarker and clinical outcome, such as diagnosis of HCC, within a particular context of use. Overall, the goals for in vitro diagnostic biomarker studies are that they should produce valid scientific evidence demonstrating reasonable assurance of the safety and effectiveness of the product, and protect the rights and welfare of study subjects $(63,64)$.

Key unmet needs in the field of chemoprevention include an improved understanding of the potential for $\mathrm{HCC}$ risk reduction by chemoprevention using commonly used medications 
such as aspirin and other antiplatelet agents, statins, metformin and similar agents(65-68). In order to build a robust evidence base through chemoprevention trials, a number of key hurdles need to be crossed, including better definition of target populations, trial enrichment or stratification prior to randomization using clinical, genetic, or other molecular risk stratifying strategies, and careful delineation of appropriate and clinically meaningful endpoints for both biomarker-based and chemoprevention trials. Enrichment of populations included in chemopreventive trials should aim to a reasonable time-to event (occurrence of $\mathrm{HCC}$ ) endpoint, certainly within the threshold of 5 years. Stratification factors for at risk populations have been outlined below and are mandatory to prevent imbalances. Finally, one of the bottlenecks of these trials is that the accepted adverse events for maximum tolerated doses (grade 3 toxicities are unacceptable) are completely different compared to those accepted for primary treatments of advanced tumors, where grade 3-4 adverse events at the level of $30-50 \%$ are common for currently accepted drug treatments.

\section{Early HCC stages: design of trials for resection, transplantation and local ablation}

Hepatic resection is the treatment of choice for patients with preserved liver function (Child's class $\mathrm{A}$, bilirubin $<1.0 \mathrm{mg} / \mathrm{dl}$, no evidence of portal hypertension) who have a solitary HCC $>2 \mathrm{~cm}$ without macrovascular invasion(3,4,69) (Fig 1, Table 4). Outcomes of ideal candidates treated following these criteria are significantly better compared with outcomes not following the guidelines (70). Recent guidelines accepted expanding criteria to include patients with HCC within Milan criteria (3). While 5-year survival rates are in the range of $70 \%$ after resection, recurrence of $\mathrm{HCC}$ is also around $70 \%$ at 5 years)(71). Early (within 2 years) recurrence is most commonly due to the appearance of preexisting undetected metastatic disease, with the most common site in the remaining liver; late recurrence is predominantly the result of de novo development of $\mathrm{HCC}$ in the remaining liver. There is, thus, a critical unmet need for therapy that can reduce the incidence of HCC recurrence after resection.. A study demonstrating benefit of retinoid administration(72) was not confirmed in a subsequent multicenter trial(73), and small studies suggesting benefit from adoptive immunotherapy(74) and I-131 lipiodol embolization of the liver remnant(75) the results of which have not been duplicated. To this point, all phase III high-quality adjuvant trials conducted so far in this area have been negative, A large randomized, controlled trial of sorafenib after resection or thermal ablation demonstrated no benefit(76). Current attention is largely focused on immunotherapy. Treatment of advanced HCC with anti-PD1 or PD-L1 antibodies has consistently yielded responses in the range of $15-20 \%((38,39,41)$ that are often quite durable. In non-small cell lung cancer similar response rates are seen in advanced disease, and a neoadjuvant trial for resectable tumors resulted in a roughly doubled response rate(77).

Phase 3 trials are currently underway with single-agent immunotherapy or combination therapies. In advanced disease combination therapy, an anti-PD-1/PD-L1 plus either a tyrosine kinase inhibitor (e.g. sorafenib, lenvatinib), an anti-VEGF antibody (e.g. bevacizumab), or a second checkpoint inhibitor (e.g., anti-CTLA-4 antibody) appears to significantly raise response rates, and if established in the advanced setting combination therapy will no doubt be studied in adjuvant/neoadjuvant trials. The ultimate hope is that effective adjuvant/neoadjuvant therapy will be able to substantially improve recurrence-free survival. It is the consensus of the panel that entry criteria for adjuvant/neoadjuvant studies in HCC resection should conform to the criteria for resectability currently espoused in AASLD guidelines $(4,69)$, and prevent a broadening of the tumor eligibility for resection (e.g., multiple tumors, presence of vascular invasion) observed in some currently-running adjuvant trials. While all patients undergoing resection for HCC have significant risk of recurrence, studies should stratify for known risk factors including tumor size $(>3 \mathrm{~cm})$, microvascular invasion, differentiation degree and serum AFP $>400 \mathrm{ng} / \mathrm{mL}$. Neoadjuvant 
studies provide a unique opportunity to better understand what factors are associated with response to immunotherapy or lack thereof. Pretreatment biopsy should be mandatory, and thorough characterization of the tumor immune microenvironment should be built into these trials.

Liver transplantation is the treatment of choice for HCC within Milan criteria in patients who are not candidates for resection (78) (Fig.1, Table 4). These criteria lead to median OS of 10 years and recurrence rate of $<20 \%$. In the US it has been accepted that patients with more extensive disease ( one nodule between $5-8 \mathrm{~cm}$, $2-3$ nodules $\leq 5 \mathrm{~cm}$ or $4-5$ nodules < $3 \mathrm{~cm}$ with sum of diameters $<8 \mathrm{~cm}$ ) down-staged to Milan criteria are acceptable for transplantation (79). Downstaging is not accepted by European guidelines, although performed in some countries such as Italy. A significant number of patients who enter the waiting list or a down-staging protocol drop out and do not ultimately undergo transplantation. Locoregional therapy using thermal ablation or transarterial chemoembolization have been the modalities traditionally applied to maintain HCC within Milan criteria while awaiting transplant or to down-stage patients to eligibility. With the advent of effective systemic therapies, their role in the pretransplant setting vis-à-vis locoregional treatment warrants exploration in clinical trials. Locoregional treatment should be the control arm, compared to systemic therapy either alone or in combination with locoregional, with the primary endpoint of drop-out / transplantability. Stratification should be according to whether patients were initially within or beyond Milan criteria, or downstaged to Milan, and base-line AFP levels $>400 \mathrm{ng} / \mathrm{ml}$.

Treatment of HCC recurrence following transplantation is largely unstudied. The rate of recurrence in properly selected patients is low (10-20\%) and these patients have been routinely excluded from studies of systemic therapies. Tyrosine kinase inhibitors have been shown to be safe and are commonly used in an uncontrolled manner(80). There is considerable reluctance to use immunotherapy with anti-PD-1/L1 antibodies due to reports of treatment-related organ rejection, though there are reports of successful treatment(81). As HCC now accounts for nearly $25 \%$ of liver transplants in the US, it is time for trials to be implemented studying treatment of post-transplant HCC recurrence.

Local ablation is the mainstay treatment for nonsurgical candidates with early stage HCC $(3,4)$ (Fig 1, Table 4). Tumor size (up to 4-5 cm), number (up to 3 tumors) and location (accessiblility with ultrasound, CT or MRI guidance) limit the applicability of percutaneous ablation. Several randomized studies have demonstrated a significant benefit of radiofrequency ablation (RFA) over percutaneous ethanol injection in terms of complete response rate, and time to recurrence $(82,83)$. Consequently, RFA is the standard ablative therapy at early stages (Table 1). Median OS with RFA is of 60 months, with a recurrence rate ranging from $50-70 \%(3,4,82,83)$. AASLD and EASL guidelines have adopted radiofrequency ablation as front line therapy for single tumors $<2 \mathrm{~cm}$, but in tumors beyond this threshold resection remains as first treatment option(3,4). Randomized phase III trials are scarce in this arena, and are mostly currently focused on adjuvant therapies to prevent recurrence than in challenging the abaltive treatment. Microwave ablation has largely supplanted RFA in the United States(84), whereas ethanol injection is restricted to $\mathrm{HCC}<$ $2 \mathrm{~cm}$ in difficult locations. Cryoablation and irreversible electroporation are still under investigation $(3,4,85)$. Clinical benefit associated with the use of thermally-sensitive carriers loaded with liposomal doxorubicin in conjunction with radiofrequency ablation is currently tested in phase III.

Overall, the main criteria for trial design in the neo-adjuvant/adjuvant after resection/local ablation or liver transplantation setting are as follows (Table 1): 
1. Target populations for neoadjuvant and adjuvant trials: For resection, trials should include patients meeting current AASLD guidelines, and should not include patients with more advanced $\mathrm{HCC}$, e.g. macrovascular invasion. For transplantation, trials should include patients meeting criteria for listing (i.e., Milan criteria), or meeting established criteria for entry into downstaging protocols. For local ablation the target population should follow AASLD guidelines.

2. Endpoints: The appropriate end-point for adjuvant trials in the setting of either resection or transplant is recurrence-free or time to recurrence. For neo-adjuvant trials, pathological response or 1-yr recurrence can also be considered. For treatments challenging locoregional therapies, OS remains the primary endpoint, but PFS is also recommended as coprimary end point. Secondary endpoints should at least include objective response rates.

3. Stratification prior to randomization: Appropriate stratification parameters for neoadjuvant/adjuvant studies in the setting of early-stage HCC should include geographical region, tumor size and number, AFP $>400 \mathrm{ng} / \mathrm{mL}$, type of curative treatment, and pathological features of high risk (size $>3 \mathrm{~cm}$, microvascular invasion, differentiation degree and tumor satellites).

4. Control arms: For neoadjuvant/adjuvant studies in the setting of resection, a placebo control arm is appropriate. Adjuvant studies in transplantation should also include placebo controls. Defining the control arm for neoadjuvant studies in transplantation remains problematic as there is no evidence-based standard, but there is a general acceptance of the need to include loco-regional therapies to limit tumor progression in patients awaiting transplant that precludes including placebo or untreated patients. Control arms for devices or drugs challenging local ablation should be radiofrequency. Of note, since RFA has been considered effective in nodules up to $4 \mathrm{~cm}$, trials exploring treatments for single nodules beyond this size should consider chemoembolization as the best standard control.

5. Unmet needs: HCC recurrence rates after resection or local ablation are unacceptably high. Key needs include biomarkers to improve case selection, and effective neoadjuvant/adjuvant therapies. With regard to transplantation for HCC key needs include definition of optimal neoadjuvant (waiting list) strategies, and identification of useful biomarkers to refine candidate selection beyond algorithms based on tumor size and number.

\section{Trial design and endpoints in intermediate stage HCC}

TACE was established as the standard of care for intermediate stage HCC in 2002 following the publication of two small, randomized controlled trials for which OS was the primary endpoint (Table 4). The first trial, conducted in Barcelona, demonstrated a hazard ratio of 0.47 [95\% Cl 0.25-0.91], p=0.025) in favor of TACE, and a 2 year survival of $63 \%$ compared with $23 \%$ for supportive care(86). In the second, TACE was associated with an improvement in 2 year survival from $11 \%$ with supportive care to $31 \%$ with TACE, and a reduction in relative risk of death; $0.49(95 \% \mathrm{Cl}, 0.29-0.81 ; \mathrm{P}=0.006)(87)$. Response using WHO criteria, was evaluated as a secondary endpoint and was shown to be associated with a better survival(86). On the basis of these trials and a subsequent meta-analysis(88), the BCLC algorithm recommends TACE for those with intermediate stage disease HCC defined by liver confined, multinodular disease, in those patients with a performance status of 0 , Child Pugh A or B cirrhosis and in the absence of portal vein invasion(3,5) (Fig 1). Chemoembolization was subsequently adopted by AASLD and EASL guidelines of management of HCC, and no other therapy has so far replaced this standard of care. However, since 2003(86,88) there have been further innovations, guidelines and therapeutic advances which need to be considered in the design of current and future trials. Finally, radioembolization with Y90 for intermediate HCC has produced positive efficacy 
signals coming from phase 2 investigations(89), but they have not been adopted by guidelines awaiting phase 3 positive data for this specific population.

Eligibility criteria and stratification factors.

It is increasingly recognized that the BCLC B stage is heterogeneous and this likely accounts for the wide spectrum of reported survival outcomes, which range from 12-48 months. Consequently, there have been several proposals to subdivide the BCLC group but to date, none have been widely adopted(90,91). Additionally, patients who have a performance status of 1 but otherwise conform to the BCLC criteria, are routinely treated with TACE, and many clinicians regard Child-Pugh $B$ disease as a relative contraindication. Applicability of TACE in BCLC-B is $50 \%$, with the excluded patients having relative contraindications for the procedure due to advanced liver dysfunction or technical issues(92). Recent large RCTs have included patients with PS $0-1$, Child-Pugh $A$, and absence of portal vein thrombosis (Table 1,2)(93-96). Stratification factors have been less consistent with the exception of AFP for which a threshold of $400 \mathrm{ng} / \mathrm{ml}$ has been commonly applied. Composite and fully objective prognostic systems may provide a more feasible and consistent method by which to stratify patients. The ALBI score allocates a grade based on bilirubin and albumin and provides a more objective measure of liver function as compared with Child-Pugh class(97). A direct comparison between ALBI and Child Pugh has shown that the ALBI grade 1 is $92 \%$ Child-Pugh A5, ALBI 2 spans a wide range from A5 to B9 and ALBI 3 is B7 and above(98). However, tumor characteristics such as size and AFP are also prognostic and this has been addressed by the HAP score which provides a four class prognostic system using bilirubin, albumin, tumour size and AFP as categorical variables(99). The HAP score has been validated in the TACE-treated population, most recently within a cohort of 3000 patients(100). Applying the HAP score resulted in four distinct groups with survival ranging from 33 months for HAP A to 12 months for HAP D. HAP appears to be a simple and robust stratification factor that might be incorporated into TACE trials

\section{$\underline{\text { TACE procedure }}$}

The TACE technique provides another source of heterogeneity and potential bias(101). There remains no consensus regarding the optimal embolic particle, the role of lipiodol or the type of chemotherapy used. Indeed, there are no trials demonstrating the superiority of TACE over bland particle embolization (TAE) and a meta-analysis of five trials including 582 patients showed no difference in survival(102). It is unlikely that further technical innovation to the TACE procedure will result in significantly improved outcomes and the future generation of TACE trials will continue to evaluate the combination of TACE and systemic therapy or to compare TACE with systemic therapy. In both cases, TACE will be the control arm and it is important that this is standardized. To achieve this, some of the recent randomized trials have mandated use of drug-eluting beads (DEB TACE)(93,94). Trials comparing DEB TACE with conventional TACE (CTACE) have failed to show a survival benefit but systemic toxicity from chemotherapy is reduced with DEB TACE(103) (104). If technique is not standardized, stratification according to center is an alternative way to reduce bias. Another area of contention is the schedule of TACE administration. In clinical practice, TACE is usually performed on demand according to radiological response rather than according to a fixed interval, and it is reasonable to recapitulate this in clinical trials. However, an effective systemic therapy may reduce the requirement for TACE. In the TACE-2 trial, there were $18 \%$ fewer TACE procedures performed in 12 months in the sorafenib arm compared with the placebo arm(93), and in the Oriental trial, the median number of procedures was 3.2 versus 3.7 in the orantinib and placebo arm respectively(96). Recording the number of procedures over the first 12 months or the mean number of procedures should be considered as a secondary endpoint for randomized trials of TACE 
versus TACE plus systemic therapy. In this sense, the reduction in frequency and number of TACE procedures may have implications for health economics and preservation of liver function.

\section{$\underline{\text { Response assessment }}$}

Radiological response is an important indicator of therapeutic activity and can be a surrogate marker of long-term outcomes. Response assessment has been addressed in the next section, but few concepts regarding loco-regional therapies are summarized here. In TACE-related population, mRECIST demonstrated a higher response rate compared with RECIST 1.1(105). Moreover, there was a significant association between survival and overall response according to $\mathrm{mRECIST}$ but not with RECIST 1.1. The association between mRECIST response and survival has subsequently been confirmed in multiple other studies and a recent meta-analysis of seven studies including 1357 patients reported a hazard ratio for survival of $0.39(95 \% \mathrm{Cl} ; 0.26,0.61)$ for those with mRECIST response(106). Unfortunately, not all the recently reported phase 3 studies reported response and only TACE-2 ascertained response by both RECIST 1.1 and mRECIST. Best response by RECIST 1.1 was higher than first response but still less than response by mRECIST. Guidelines recommend capturing response as per mRECIST in clinical practice and both RECIST 1.1 and mRECIST as secondary endpoints trials targeting intermediate stage tumors(3).

\section{Primary endpoints}

In recent trials, OS for intermediate stage patients receiving TACE was of 21-33 months(9396) (Table 2). Over the past 10 years, there have been major advances in systemic therapy and many patients now transition from TACE to first and increasingly second line systemic therapy. In TACE-2, patients were unblinded on progression and $36 \%$ of those on placebo subsequently received sorafenib(93). Similarly, in the BRISK TA trial, $21 \%$ of placebo treated patients had post-progression systemic therapy (95) trial, and in the ORIENTAL trial, $66 \%$ of patients in the placebo arm received post-study therapy (96). Use of postprogression therapy may confound OS as an endpoint and increases the duration of followup required to meet the survival endpoint. To address this, a variety of surrogate endpoints have been proposed including progression free survival (PFS), time to progression (TTP), time to disease progression (TTDP), time to extrahepatic spread and vascular invasion (TTES/VI) and time to unTACEable progression (TTUP). Recent trials reporting these potential surrogates in addition to survival has allowed evaluation of their performance. The BRISK TA trial reported a promising hazard ratio of 0.61 for TTP but the trial missed its primary endpoint for survival (HR 0.9)(95). Overall, the correlation coefficient of TTP and OS is 0.77. A major limitation of TTP is that it fails to capture death, which is an important indication of toxicity as well as lack of efficacy. By contrast, PFS, which is the most commonly applied surrogate endpoint used in oncology, captures disease progression and death, and has been reported to correlate with OS in the TACE 2 trial. Novel composite endpoints have also been explored. Time to appearance of extrahepatic spread or vascular invasion (TTES/VI or MVI/EHS) showed a promising HRs of 0.64 and 0.62 in the BRISKT TA and SPACE trial that did not correlate with OS benefit(94,95). Particularly, TTUP (time to untreatable progression), a composite end point defined as failure of response after to treatments, or emerging contraindications for TACE was tested in the SPACE trial, but failed to identified benefits for the combo of TACE plus sorafenib vs TACE (HR: 1.586). Recently, other novel endpoints were incorporated into the TACTICS trial comparing TACE plus sorafenib vs TACE alone(107) (108). In this study, PFS and OS were co-primary end points but progression was defined as unTACEable progression and Response Evaluation Criteria in Cancer of the Liver (RECICL)(109) was used to define progression rather than RECSIT 1.1 or mRECIST. Applying these criteria, PFS was superior in the combination arm 
$(\mathrm{HR}=0.59 ; 95 \% \mathrm{Cl}, 0.41$ to $0.87 ; \mathrm{p}=0.006)$ but further follow-up is required to establish wether this translates into a survival benefit. In the meantime, for RCT testing devices alone or in combination with systemic therapies it is recommended that PFS should be the coprimary endpoint along with OS, while ORR should be included as a secondary endpoint (Table 1 \& 2). Additional composite endpoints can be included as exploratory endpoints until they are properly validated.

A challenging question for the future is how TACE compares to systemic therapy. TACE was developed at a time when systemic therapy was virtually non-existent. With the advent of first, second and even third line systemic therapies and achieved OS beyond 2 years in selected patients receiving two lines of therapy(23), systemic therapy can be discussed not only following TACE but as an alternative to TACE. This is particularly relevant as transarterial therapies impair liver function and may render many patients no longer eligible for systemic therapy. For patients with limited tumor burden and nodules accessible superselectively by TACE, locoregional TACE may still be the best approach. In contrast, patients exceeding the up-to-seven criteria may be better suited for clinical trials exploring upfront systemic therapy(110). To answer this question a head-to-head comparison of TACE vs. systemic therapy (or vs. TACE plus systemic therapy) in defined patient subgroups will be needed, making the endpoint discussion even more complex.

\section{Radiologic assessment of response}

The RECIST criteria are the standard imaging approach for assessing tumor response in oncology. The original RECIST panel acknowledged that amendments could be needed for tumors with unique complexities and for evaluating non-cytotoxic drugs(111). Both issues are highly relevant for HCC: (a) the association of HCC with an underlying chronic liver disease complicates image assessment, since pathologic and hemodynamic changes in cirrhosis and extrahepatic manifestations of chronic liver disease may mimic tumor progression; (b) nonsurgical treatments for HCC, including loco-regional and systemic therapies, achieve improvements in survival without inducing sizeable tumor shrinkage, frustrating attempts to capture tumor response using standard RECIST metrics(12).

In 2010, modified RECIST (mRECIST) criteria for HCC were proposed(21) addressing confounding factors related to cirrhosis using specific amendments for the assessment of lymph nodes, ascites, portal vein thrombosis, and newly detected hepatic nodules (Table 5). These recommendations were made primarily to prevent "overcalls" of progressive disease. In addition, the absence of substantial tumor shrinkage was addressed by introducing the concept of "viable tumor" in the measurement of intrahepatic HCC lesions, enabling the classification of treatment induced intratumoral necrosis in the absence of significant changes in overall tumor diameter as objective responses (21).

During the past decade, mRECIST for HCC has been used extensively in HCC clinical research and its performance has been reviewed elsewhere(27). The proposed mRECIST refinements for assessment of lymph nodes, ascites, portal vein thrombosis, and newly detected hepatic nodules, were progressively incorporated into radiology charters of HCC clinical trials, even when the criteria were named RECIST or RECIST 1.1(112). This process homogenized radiologic interpretation of these findings, improving consistency and reliability in assessment of tumor progression. Consequently, recent studies reported similar results for standard RECIST 1.1 and mRECIST in assessment of progression-driven endpoints, such as PFS and TTP $(7,8)$. Currently, the main difference between standard RECIST and mRECIST is the approach to measurement of intrahepatic lesions, which primarily affects the ability to capture an objective response (OR). Use of the mRECIST viable tumor concept results in identification of 2-3 times more responders than standard 
RECIST, not only in patients receiving loco-regional treatments but also those receiving systemic therapies(7,32).

With the advent of immune checkpoint inhibitors, changes to the RECIST model have been proposed(35,36,113-115). Response to immunotherapy can manifest after imaging features that meet current RECIST criteria for progression. Pseudo-progression has been defined as increase in tumor size of existing lesions or the appearance of new lesions, followed by a response(35). Differentiating pseudo-progression from true progression is a challenging but important: while early discontinuation of an effective drug is not desirable, continued long-term treatment with a non-effective drug past true progression might delay the initiation of potentially effective therapies. Pseudo-progression has been described as a marginal event in phase III investigations with anti PDL1/PD1 check point inhibitors in HCC. The incidence of this phenomenon with anti- CTLA-4 and other inhibitors is unknown.

Limited information is available on use of immune-related criteria in HCC. In a phase II study of 104 patients who received pembrolizumab in second line after sorafenib, the use of immune-related RECIST (irRECIST) did not affect response rate or time to response as compared to mRECIST; however median PFS was 7.0 months $(95 \% \mathrm{Cl}, 4.9-8.0)$ when assessed by irRECIST vs 3.2 months (95\% CI, 2.2-4.1) when assessed by mRECIST(116). In phase Ilb study(117) investigating a vaccinia virus-based oncolytic immunotherapy pexastimogene devacirepvec- in advanced HCC changes to $\mathrm{mRECIST}$ were implemented because the treatment induces a flare with swelling and edema(118). These changes included the confirmation of progression at 4 weeks, either by further increase in size or additional signs of progression such as emergence of new lesions(117). Overall, to assess response to checkpoint inhibitors or immunotherapies in HCC, evaluation by CT/MRI at 812 weeks after treatment can be recommended, as opposed to the usual interval of 6-8 weeks for tyrosine kinase inhibitors. This window was used in phase II studies testing nivolumab (12 weeks)(38) and pembrolizumab (9 weeks)(116), where the phenomenon of pseudo-progression was reported as a marginal event.

\section{Design and endpoints for systemic therapies in HCC}

\section{Standard of care with systemic therapies in HCC}

Current estimates suggest that around $50 \%$ of HCC patients will receive effective systemic therapies during their lifespan $(3,119,120)$. Several trials have tried to show survival benefits of systemic agents in advanced disease (Table 2,4), a traditionally challenging setting due to the limited efficacy and high toxicity of conventional systemic chemotherapy(121-124). Randomized studies for anti-estrogen therapies also failed to prove any clinical efficacy (125). In 2008, the landmark SHARP trial assessing the multi-tyrosine kinase inhibitor sorafenib was the first to significantly improve survival with manageable adverse events(11). Afterwards, five treatments have succeeded, while several other drugs failed $(126,127),(22),(128)(129)(122)(130)(131)(132)(133)-$. In first line, atezolizumab (anti-PD-L1 inhibitor) plus bevacizumab (VEGFA inhibitor) have shown to be superior to sorafenib in a recently reported $\mathrm{RCT}(134)$. The study was stopped at the first interim analysis by showing a HR of 0.58 for OS (median not reached for combo vs 13.2 mo for sorafenib) and HR of 0.59 for PFS (median 6.8 mo for combo vs 4.3 for sorafenib). These results will pose this combination as standard of care first-line therapy for advanced HCC. Second, lenvatinib (multikinase inhibitor: VEGFRs, FGFRs, RET, KIT and PDGFRA) has become an option equal to sorafenib, after the positive result of the non-inferiority REFLECT study (HR of 0.92; 95\% Cl 0.79-1.06) (Table 2, Fig 2A). Because this trial excludes patients with main portal vein invasion, tumor involvement $>50 \%$ of the liver and clear bile duct invasion, the relative benefit of lenvatinib vs sorafenib in these patients remain uncertain. 
In second line, the phase III trial testing regorafenib (VEGFRs, PDGFRs, KIT and Tie2) improved OS compared to placebo from 7.8 to 10.6 months (HR of 0.63 ) in patients who progressed and were tolerant to sorafenib(8). The sequential treatment sorafenibregorafenib led to a median OS of 26 months compared to 19 months for sorafenib-placebo (23). These results need to be taken with caution since they will not apply to all patients receiving sorafenib, but only those able to receive the sequential treatment. The CELESTIAL study, showed median OS of 10.2 months with cabozantinib (VEGFRs, MET and $A X L)$ vs. 8 months with placebo (HR of 0.76$)(9)$; and the REACH-2 study, where ramucirumab (VEGFR2 monoclonal antibody) provided a median OS of 8.5 months in patients with AFP $\geq 400 \mathrm{ng} / \mathrm{ml}$ vs. 7.3 months with placebo (HR of 0.71$)(10,135)$. AFP is well-known for its independent prognostic capacity in $\mathrm{HCC}(136)$. As such, REACH-2 was the first and only positive phase III trial in a biomarker-driven population of patients with HCC (Fig 2B). In contrast, 3 phase III trials testing internal radiation with $Y-90$ for advanced HCC, either as single treatment [SARAH(137) and SIRveNIB(138)] or in combination Y-90 with sorafenib(139) did not meet the primary endpoint of improved OS compared to sorafenib (Fig. 2A). As a result, Y-90 was discouraged for the management of advanced HCC in the EASL guidelines (Fig. 1)(3). Despite appealing ORR of $15 \%$ with durable response for nivolumab and $18 \%$ for pembrolizumab, phase III trials comparing the former with sorafenib(24) in front-line and the latter with placebo in second-line resulted negative. Particularly, the latter trial showed a HR of 0.78 with upper boundary of $95 \mathrm{Cl}$ below 1 , but the pre-specified $p$ value $(p<0.0178)$ was not hit(41).

\section{Trial design in advanced HCC}

Overall survival remains as the primary end-point for advanced HCC research $(1,3)$ (Table 4). It has driven clinical research in HCC for more than 40 years and has been the goldstandard for measuring benefits at all stages of the disease. Nonetheless, the emergence of several effective drugs in advanced $\mathrm{HCC}$ has exposed the need for alternative end-points that can capture the benefits of a treatment before they can be diluted by post-progression therapy(3). Progression-free survival (PFS), time to progression (TTP) and objective response rate (ORR) are now emerging as tools to a) identify early strong signals of efficacy that led to accelerated regulatory approval (particularly ORR and PFS) $(6,88)$ and b) test interventions which benefit can be assessed prior additional sequential drugs received beyond progression might mask the actual benefit of the tested drug. In this sense, a recent investigation analyzing 21 reported phase III studies(7-11,121-123,126$131,133,135,137,138,140$ ) in advanced HCC proposed PFS (with a restrictive hazard ratio criteria $<=0.6$ ) as a surrogate end-point for survival when testing kinase inhibitors or monoclonal antibodies, and thus as potential primary end-point in advanced HCC trials(3) (Table 4). Subsequently, six phase III studies have been released that confirm the hypothesis: two positive studies, one testing atezolizumab plus bevacizumab vs sorafenib(134) and the second sorafenib plus hepatic arterial infusion of Folfox vs sorafenib(141), both show HR for PFS $\leq 0.6$ and significant survival benefits, and four negative trials for survival testing nivolumab(24), sorafenib plus pravastatin(142), sorafenib plus doxorubicin(143) and pembrolizumab(41), in which the HR for PFS in all cases was $>0.6$ (Fig 3). Considering the special circumstances of the 2 negative trials testing anti-PD1 inhibitors, we should be cautious when applying this rule for testing immune therapies as single agents or for combinations of immune regimes.

Trial design in HCC has been evolving, and new challenges emerge as novel therapies become standard of care. Although there might be distinct approaches to trial design in $\mathrm{HCC}$, there has been a consensus on the basic principles that have been recently reported in guidelines and critical appraisals $(3,139,144,145)$. The key points are summarized below (Table 1): 
1. Phase II and Phase III trials: The panel recommends assessing drugs in the setting of randomized phase II studies before moving to phase III trials. Nonetheless, for some therapies, a large single arm phase II with a strong signal of efficacy might suffice to justify a phase III study. Thresholds for defining signals of efficacy are not clearly established, but for molecular therapies the ORR should likely be above $20-30 \%(146)$.

2. Selection of the target population: Clinical trials should consider BCLC staging system, Child-Pugh class and ECOG performance status for selection of the target population. In principle, for advanced HCC almost all RCT include patients with wellpreserved liver function (Child-Pugh A) and good performance status (ECOG 0 and 1).

3. Control arm: The control arm of randomized phase II and III studies should be the standard of care established according to guidelines. Although sorafenib and lenvatinib in front-line $(7,11)$ and regorafenib (8), cabozantinib(9) and ramucirumab (in patients with AFP $\geq 400 \mathrm{ng} / \mathrm{ml})(10)$ are accepted as standard of care, this will change when atezolizumab plus bevacizumab are approved by regulatory agencies. At that time, this combination will become the standard of care for comparison in front-line, and subsequence lines of therapy will move downwards. Double-blind trials (as opposed to open label trials) are recommended to prevent selection and allocation biases.

4. Stratification for prognostic factors prior to randomization: Stratification is critical in randomized studies to warrant balanced comparisons. For advanced $\mathrm{HCC}$ the recommendation is as follows: region, macrovascular invasion, extrahepatic spread, AFP > $400 \mathrm{ng} / \mathrm{ml}$ and ECOG 0 vs 1-2. Etiology should also be considered as studies with sorafenib and atezolizumab and bevacizumab suggest an influence of this factor in response.

5. End points: Overall survival: For systemic therapies the primary endpoint should be OS, and PFS is proposed as co-primary endpoint. To date, all regular FDA and EMA drug conventional approvals in advanced HCC were based upon improvements in OS. Surrogate endpoints: OS has limitations as a sole endpoint in cancer research: it might require a long follow-up to capture adequate numbers and can be affected by sequential therapies. Thus, surrogate endpoints that are more practical for trial execution are needed. There are no optimal surrogate endpoints able to recapitulate OS in HCC, and thus clinical practice guidelines do not recommend ORR, TTP and PFS as primary endpoints in phase III investigations(144,145). ORR is an independent predictor of OS in three phase II and III trials $(7,123,127)$, but is still considered a suboptimal primary end-point for phase III investigations. Nonetheless, ORR of $16-18 \%$ resulted in accelerated FDA approval of nivolumab and pembrolizumab in second line treatment of advanced $\mathrm{HCC}(38,39)$. PFS was formerly discarded as a primary end-point of phase III investigations due to the concept of competing risk of survival (competing between death due to tumor progression and due to the natural history of cirrhosis)(12). However, this competing risk drawback has been reduced by the universal selection of Child-Pugh $A$ patients for these investigations, thus reducing the $1-y r$ risk of death due to decompensation to $<5 \%$. Stringent criteria for accepting PFS as primary endpoint have been proposed $(\mathrm{HR} \leq 0.6)$ and it is adopted in the current guidelines (Table 1), but this point is still controversial. Regarding ORR, use of both RECIST1.1. and mRECIST are proposed for the assessment of response in HCC treated with systemic therapy, whereas changes in serum biomarker levels (i.e. AFP levels) are not supported(3).

6. Magnitude of benefit: In HCC, there is no consensus on what absolute survival benefit (or magnitude of benefit in OS according to HR) is clinically relevant. Reported thresholds of OS with HR $<0.8$ are sound for capturing the benefit of patients in advanced $\mathrm{HCC}$ trials(20). This figure needs to be taken with caution, since other variables can impact 
the overall benefit of a given drug, such as quality of life, safety profile and availability of alternative therapies in distinct countries.

7. Checkpoint inhibitors and other immunotherapies have unique features and generally produce higher ORR and longer duration of response, as measured by RECIST1.1. The values of mRECIST and irRECIST in assessing checkpoint inhibitor mediated responses remain investigational.

\section{Immune treatments: Overview of results andspecific endpoints}

The initial clinical experience with checkpoint inhibitors in HCC was with a phase II study testing tremelimumab, a CTLA-4 antibody leading to objective response of $18 \%$ of patients and time to tumor progression was 6.5 months (147). Immunotherapy has drawn significant attention in HCC with the approval of nivolumab and pembrolizumab by the FDA based on promising results obtained in different phase II studies $(38,39)$. A phase I/II open-label, noncomparative trial (CheckMate 040) assessing the efficacy of nivolumab in advanced HCC reported objective response rate (ORR) of $20 \%$ in the dose-expansion phase $(n=214)$ and $15 \%$ in the dose-escalation phase $(n=48)$. Duration of response (DOR) was 9.9 months and median PFS as 4.0 months in the dose-expansion cohort. Nivolumab treatment was well tolerated (38). Pembrolizumab, another PD1 specific antibody, was tested in phase II in patients with HCC progressing or intolerant to sorafenib (Keynote 224). Pembrolizumab was effective and tolerable with one complete response $(C R)$ and 17 PR out of 104 patients. The median progression free survival was 4.9 months, and median OS was 12.9 months(39). Camrelizumab, another fully humanized anti-PD-1 antibody, was evaluated in a randomized phase 2 trial in Chinese patients with advanced HCC after failure of at least one line of therapy(148). The ORR was $13.8 \%$ and the 6 -month OS was $74.7 \%$.

Nivolumab and pembrolizumab failed in phase III trials (Fig. 2A-B). Pembrolizumab was tested in a randomized, double-blind phase III trial against placebo in 443 patients with HCC that progressed on or were intolerant to sorafenib (Keynote-240). The co-primary endpoints of PFS and OS failed to reach the prespecified level of statistical significance although median OS was prolonged from 10.6 to 13.9 months (HR: $0.781 ; 95 \% \mathrm{Cl}: 0.611-0.998 ; \mathrm{P}=$ .0238)(149). Nivolumab was tested against sorafenib in a Phase 3 trial (CheckMate 459), but did not reach survival differences for superiority (24). In this RCT including around 750 patients, median OS for nivolumab was $16.4 \mathrm{mo}$ vs $14.7 \mathrm{mo}$ for the sorafenib arm (HR: 0.85 ; $95 \% \mathrm{Cl} 0.72-1.02$ ). Objective response rate was $15 \%$ and $7 \%$, respectively.

Anti-CTLA4 antibodies have been tested as single agent(147) or in combination with locoregional therapies(150) and are under investigation in combination with anti-PD1 drugs(151). In this regard, very recently the combination ipilimumab and nivolumab received FDA approval based on a ORR of $31 \%$ (40). Currently phase III trials are ongoing which either test the combination of two immune checkpoint inhibitors, immune checkpoint inhibitors plus TACE, immune checkpoint blockade in the adjuvant setting or immune checkpoint inhibitors plus vascular targeting agents(152). While the overall response to immune checkpoint inhibition (15-20\%) may not be as dramatic as initially hoped, complete responses are seen in a small number of cases in almost every trial. This observation together with the recent results from two phase III trial testing anti-PD1 antibodies in the first- and second-line setting rise up the important question of what endpoint to use in future trials. While OS remains the "gold standard" it should be noted that HCC is not the only cancer where this question is being asked. Due to the unique mechanism of action of immune checkpoint inhibitors(153), new endpoints such as ORR and surrogate biomarkers have been tested and new immune related RECIST criteria devised to capture distinctive 
patterns and timing of response to immunotherapy $(35,115)$. Finally, while immunological endpoints may be helpful as surrogates(154), they cannot be recommended at this time.

A systematic review and meta-analysis of 87 phase 2 trials with the foal of defining the most appropriate primary endpoint in phase 2 trials of immune checkpoint inhibitors for advanced solid cancers has been reported. Correlations between ORR odds ratios and hazard ratios (HRs) for PFS and OS were examined for randomized comparisons. Within checkpointinhibitor treatment arms, correlations of ORR with 6-month PFS and 12-month OS rates were examined. All analyses were weighted by trial size. Multivariable models to predict 6month PFS and 12-month OS rates from ORR were developed and their performance validated in an independent sample of trials. The authors demonstrated that ORR correlated poorly with OS and recommended 6-month PFS rate as an endpoint for future phase 2 studies,(155). Thus, PFS endpoint can also be recommended for studies evaluating immune checkpoint inhibitors in HCC (Table 1).

\section{Biomarker-Driven Trials in HCC: Results and Endpoints}

Recent clinical trials in advanced HCC are demonstrating that the sequential use of systemic agents is changing the natural history of the disease. Still, these results are incremental and the incorporation of biomarker driven strategies have generally been unsuccessful. Unlike other solid tumors such as breast, lung, colon cancer, and others, where therapeutic decisions are driven by an understanding of a patient given molecular features, in HCC a "one-size-fits-all approach" is still the usual approach to patients. This applies to all therapies so far accepted in guidelines, except for ramucirumab.

Biomarkers provide the distinct possibility of supplementing existing anatomic and/or pathologic information to provide a more accurate assessment of prognosis (to be used for patient stratification) and/or to identify individuals who are more likely to respond to specific therapy (predictive of response) (156-158) There is a plethora of literature on the different predictive biomarker validation designs (159). The $\mathrm{NCl}$ defines a biomarker as a biological molecule found in blood, other body fluids, or tissues that is a sign of a normal or abnormal process, or of a condition or disease. A biomarker may be used to determine how well the body responds to a treatment for a disease or condition (160).

In HCC, numerous studies have defined the molecular heterogeneity of the disease and specific genetic alterations and subtypes. These data are fertile ground for testing biomarker hypotheses as both prognostic and/ or predictive markers in prospective studies but so far these data have largely been ignored in clinical development in HCC (6). To date, two phase 3 studies have tested biomarker driven approaches. Firstly, tivantinib, a small molecule inhibitor of the hepatocyte growth factor/ c-MET was evaluated in patients that had progressed on sorafenib and had elevated expression of c-MET in their tissue. This was a placebo-controlled study that yielded negative results(131). The possible reasons for failure highlight the challenges with this approach including: 1) validity of the target, 2) robustness of the assay for patient selection, and 3) ability of the agent to inhibit the target successfully in tumor tissue. The latter may be a plausible reason for failure of the trial, considering that the anti-MET activity of this drug has been challenged in experimental studies(161). Conversely, ramucirumab, which initially failed in an "all comers" study(162), demonstrated an improvement in OS for selected patients with AFP $\geq 400 \mathrm{ng} / \mathrm{ml}$. Proof-of-concept studies testing small molecule inhibitors of FGFR-4 using biomarker enriched populations based on FGF-19 expression have been reported with ORR of 16\% (163).

Recently, immunotherapy approaches have garnered high interest in the management of HCC and the PD-1 directed antibodies nivolumab and pembrolizumab received accelerated approval by the US FDA $(38,39)$. However, unlike in other cancers, PD-1 and / or PD-L1 
expression has not correlated with outcome. This has likely contributed to the recent negative results from phase 3 studies with these agents(149). Ongoing work is focused on further refining biomarker development evaluating other inflammatory markers including incorporation of more broad based assessment tools such as an immune-enriched signature identified through molecular profiling of HCC (164).

Several studies have incorporated biomarker assessments into the trial design. While tissue collection is often optional and therefore limited, serum assays have served to generate hypotheses for further study. In the pivotal SHARP study, baseline levels of angiopoietin 2 and VEGF were prognostic but not predictive of benefit from sorafenib(136). Relevant biomarkers in the FGF and VEGF pathways were analyzed in the REFLECT study and identified differences in the modulation of these pathways by lenvatinib and sorafenib, but no biomarker could define a group receiving differential benefit from either compound(165). In the REACH-2 study, decreases in AFP correlated with better outcome from ramucirumab(10). Novel study designs evaluating biomarker assessments pre-and posttreatment are being performed. These so-called "pre-surgical" studies are designed to acquire tissue at baseline, from patients with resectable tumors, expose the patient to a novel agent for a short period, and then collect tissue at the time of resection. Molecular studies comparing the pre-and post-treatment tissue provide an opportunity to understand the effects of novel therapeutics on relevant pathways in the tumor. These studies can provide critical information that could guide a patient selection strategy in conventional efficacy studies. One such study with nivolumab is producing interesting insights into tumor characteristics that may correlate to response to this drug(166).

Despite the recent successes in clinical trials of new agents for HCC, the improvements in survival are modest. Throughout cancer medicine, the largest impacts in outcomes have been by biomarker driven drug development. Examples include ALK(167) and EGFR(168) testing in lung cancer, HER-2(169) and estrogen receptor(170) testing in breast cancer, cKIT testing in gastrointestinal tumors(171), and BCR-ABL testing in chronic myelogenous leukemia(172). By enriching for the population most likely to benefit, studies can be conducted with smaller numbers of patients and minimize risk for failure. While historically predictive marker testing is done on tumor tissue obtained by biopsy, newer technologies are now allowing biomarker detection in peripheral blood. The practice of not obtaining biopsies for diagnosis of HCC, the fact that most common driver mutations in HCC are nonactionable and the observation that only $25 \%$ of HCCs harbor at least one actionable mutation(172), in contrast to the majority of solid tumors(173), have hindered development of biomarker driven precision treatment to date. Nonetheless, there is now renewed interest in incorporating tissue acquisition into clinical trials, not only in the early part of drug development, but in later studies as well. Clinical trial designs for predictive marker validation are inherently complex and require data from a randomized controlled clinical trial $(\mathrm{RCT})(153)$. There is a plethora of literature on the different predictive biomarker validation designs, including articles that specifically focus on the statistical and clinical properties and assumptions of these different trial designs (156).

Trial design in the precision medicine era require a platform for biomarker profiling (173)(174). The ultimate clinical utility of a biomarker will depend on: 1) its added value in every patient in the context of the markers prevalence, 2) its incremental benefit for treatment selection when considering the added costs and complexity induced by the use of the marker, and 3 ) the added effectiveness of the new treatment option in all patients versus biomarker-defined subgroups. 


\section{Liquid biopsy in early HCC detection, prediction of response and tumor relapse}

Liquid biopsy entails the analysis of tumor components released by cancer cells to biological fluids such as blood, saliva or cerebrospinal fluid(175). The concept includes the analysis of actual cancer cells (i.e., circulating tumor cells [CTCs]), fragments of DNA from necrotic or apoptotic cancer cells (i.e., circulating tumor DNA [ctDNA]) and extracellular vesicles(176). Compared to conventional tissue biopsies, the main advantages of liquid biopsy using samples from peripheral blood are: 1) it is minimally invasive, which eliminates the complications associated with invasive tissue biopsies; 2) it facilitates sequential sampling, which is crucial to better select therapies in patients receiving multiple lines of treatment; 3 ) it enables tracking tumor clonal composition in heterogeneous tumors, a feature that allows earlier detection mechanisms of treatment resistance; and 4) it can be implemented as a point-of-care diagnostic. Potential clinical applications include cancer surveillance, early detection of minimal residual disease after curative therapies, prognostic prediction and molecular monitoring of therapeutic response(177). In an early sign of impact on patient care, the FDA recently approved the use of a ctDNA-based test to detect mutations of EGFR in lung cancer patients who are candidates to receive EGFR-based tyrosine kinase inhibitors(178).

In HCC, liquid biopsy has been evaluated for three clinical applications: early HCC detection in the context of surveillance, as a prognostic biomarker after surgical resection and to predict response to systemic therapies. Mutation profiling of ctDNA is feasible and confidently detects tissue mutations in early stage $\mathrm{HCC}(179)$. A recent report combining data from ctDNA and protein markers had a sensitivity and specificity of $85 \%$ and $93 \%$ respectively for the detection of $\mathrm{HCC}(180)$. Also, analysis of DNA methylation alterations in ctDNA has high accuracy for HCC diagnosis $(55,181)$. A study that included a gene signature derived from CTCs was able to accurately discriminate between HCC patients and controls(182). Higher CTC count correlates with a greater risk of tumor recurrence after surgical resection(175). There are few studies using liquid biopsy to predict response to systemic therapies in HCC. A retrospective study suggested that HCC patients with ctDNA detectable VEGFA DNA amplifications have better outcomes when treated with sorafenib(183). Also, RAS mutations analysis of ctDNA was used to determine eligibility to receive refametinib in a phase 2 clinical trial(184). Thus, there is increasing interest in applying this technology to predict response to systemic therapies.

\section{Quality of life and patient reported outcomes}

Systematic capture of the patient perspective can inform the development of new cancer therapies. The U.S. FDA Office of Hematology and Oncology Products (OHOP) has identified symptomatic adverse events (AEs) as a central Patient Reported Outcome (PRO) using the National Cancer Institute's Patient-Reported Outcomes version of the Common Terminology Criteria for Adverse Events (PRO-CTCAE) to provide a standard yet flexible method to assess symptomatic AEs from the patient perspective $(194,195)$. The FDA's patient focused drug development program has ongoing efforts to improve methods around the collection, analysis and interpretation of PRO data, as well as initiatives to identify patient-friendly language, and leveraging digital health tools. In 2016, the 21st Century Cures Act tasked the FDA to consider the patient-experience in the risk-benefit determination. The FDA draft guidance outlines the use of PRO to assess symptomatic side effects and the core set of clinical outcomes to measure in cancer trials, including design considerations and assessment frequency(11).

The purpose of measuring quality of life (QOL) should be to compare outcomes between treatment arms, even if one is a placebo. There are two methods of measuring QOL specific to HCC: the European Organization for Research and Treatment of Cancer Quality of Live 
Questionnaire (EORTC QLQ)(185) and the Functional Assessment of Cancer TherapyHepatobiliary (FACT-Hep)(186) questionnaire. Few studies have adequately assessed PRO using these tools in HCC research, a recommendation that is endorsed by the panel.

Most phase III trials for HCC were designed primarily to compare two different treatments in patients with similar stage disease. For example, one study compared QOL after resection with QOL following radiofrequency ablation(187). As expected, QOL was much better after radiofrequency ablation than after resection, and remained superior up to 36 months post-treatment. In addition, QOL following radioembolization has been compared with TACE(188). In this study, there was no overall difference in QOL between the two groups, but the sample size was small. Despite the lack of statistically significant differences, in the TACE group QOL was decreased at 2 and 4 weeks, whereas in the radioembolization group some aspects of QOL actually improved. Similarly, QOL measures favoring Y-90 vs sorafenib have been claimed to support the former treatment in three negative RCT, the SARAH trial (134) and the SIRveNIB trial (135) and SORAMIC trials. However, since indication of a drug/device should be based upon the primary endpoint (survival), no actual indication can be claimed if the result is negative for the primary endpoint. Finally, the SHARP trial demonstrating survival benefit of sorafenib also tested timeto-symptomatic progression - as measured by the Functional Assessment of Cancer Therapy-Hepatobiliary Symptom Index 8 (FHSI8) - as a co-primary endpoint. The negative results of this end-point contrasted with the survival benefit obtained by sorafenib, thus challenging the accuracy of the tool used(11). More recently, patient reported outcomes have been tested in the setting of phase III investigations showing significant results in positive RCT in advanced HCC. This is the case of lenvatinib compared to sorafenib, or atezolizumab plus bevacizumab compared to sorafenib, where the tested arms showed better QoL parameters compared with the standard of care. The panel encourage the integration of these endpoints in all investigations in HCC (Table 6).

\section{Implications of trial design in Asia}

\section{Differences between AASLD, EASL, and Asian guidelines}

Recommendations in western guidelines (AASLD and EASL) are based upon evidence from clinical trials (Table 4), while Asian guidelines integrate evidence with expert consensus and clinical practice. Applicability of those guidelines varies according to region and treatment stage (47). Asian guidelines (189) in general recommend ablation or resection for very early-stage (stage 0 ) disease, but differ from western guidelines in the recommendations at other stages of disease. For instance, TACE or yttrium-90 (Y90-SIRT) are recommended for single large tumors, and systemic therapies -i.e Folfox(105), or hepatic arterial infusion chemotherapy - are recommended for advanced stages, along with liver transplantation - mostly living donor transplantation. Similarly, in Asia patients with portal vein invasion and well-preserved liver function might be considered for TACE, resection or radiotherapy (190-194).

\section{Specificities of trial design in Asia}

Considering all these guidelines, trial design in Asia has some specificities. For instance, resection in very high-risk patients (multinodular tumors, macrovascular invasion) is common in Asia, and thus adjuvant trials might consider this indication with a recurrencefree survival endpoint. Similarly, studies exploring the role of systemic therapies plus TACE in patients with advanced stages might also be considered in Asia with a primary end point of PFS. Weather these approaches should be tested in specific trials or as part of global trials needs further consideration. 


\section{Future prospects}

\section{The dawn of a new era: combination therapies}

When the report of the first AASLD conference on Design and Endpoints of Clinical Trials in HCC was published(12), the field was still heady with excitement from the first positive trial of a systemic agent for advanced HCC, which established sorafenib as the first FDA approved systemic therapy for HCC (11). However, enthusiasm was also tempered by the subsequent negative results of trials of sorafenib as adjuvant therapy after resection or ablation (STORM)(76) or in combination with TACE (SPACE)(94). It was recognized then that a unique challenge is posed by the combination of underlying chronic liver disease with a very heterogeneous and variably aggressive primary HCC. It is therefore important that treatment strategies account for both the liver disease and malignancy, and thus variables capturing both diseases should be considered in the publication of clinical trials for HCC (Table 7). Discussions at the previous AASLD Endpoints conference set the framework for subsequent attempts to bring additional agents to approval, which were met with uniformly disappointing results over the next several years, with trial failures due to unacceptable toxicity or inadequate efficacy(12). While disappointing, these failures led to robust examination of the optimal approach to trial design and catalyzed a more rigorous approach that contributed to the successes in phase $3 \mathrm{HCC}$ clinical trials. With the positive results and approvals of lenvatinib, regorafenib, cabozantinib and ramucirumab based on phase 3 studies, and of the checkpoint inhibitors nivolumab and pembrolizumab based on convincing phase 2 data, we appear to be poised for success in the next most logical treatment paradigms using combination therapies. Indeed, the recent positive phase III study demonstrating superior OS for atezolizumab plus bevacizumab vs sorafenib(134), represents the dawn of a new era of combination therapies in all stages of HCC research (Fig 1). This combination is certainly first in-class of this approach. Whether other combinations may become best-in-class will depend upon the ability of specific TKI and/or MAb to transform "cold tumors", which are primary resistant to immunotherapy, into "hotimmune-active tumors", allowing checkpoint inhibitors to optimally unleash immune attack against cancer cells(195-197) .

The advent of combination therapies achieving response rates of $30 \%$ and survival rates above 20 months in advanced HCC provides the rationale for testing these combinations in earlier HCC stages. Currently, phase III trials testing combination therapies are ongoing for early stages (neo-adjuvant or adjuvant approaches), intermediate $\mathrm{HCC}$ (in combination with TACE or in comparison to TACE) and in front-line trials for advanced HCC (Table 7). It is conceivable that systemic therapies may be incorporated in all areas of HCC management in the near future. Thus, the up-dated target population and endpoints described here should be valuable in this endeavor.

\section{Understanding tumor biology remains critical: tissue and blood samples are needed}

It is likely that the next key advances in HCC therapy will emanate from an improved understanding of HCC biology and the ability to predict the response of specific HCCs subgroups to particular therapies. Until now, most HCC therapy has been applied in a biologically indiscriminate fashion. The biological heterogeneity of HCC has been evident for many years, demonstrated by differences in phenotypes, tumor growth rates, numbers of tumor nodules, discrete versus infiltrative appearance, propensity for microvascular or macrovascular invasion, propensity for distant metastasis, and association with elevation of AFP, AFP-L3, DCP and other biomarkers. Apart from the limitations that multifocal, invasive or metastatic disease have placed on application of potentially curative treatments such as surgical resection, liver transplantation, and local ablation, we have only recently began to incorporate markers of tumor biology into therapeutic decision making. Applications of tumor 
biologic characteristics into therapeutic approaches have been scarce in HCC and mostly focused on using AFP levels for selection policy for transplantation, as an stratification factor in most of trials and finally for selecting candidates to ramucirumab in the management of advanced HCCs in second line.

With the advent of next generation DNA, RNA and non-coding RNA sequencing and similar genome wide methodologies for copy number variation, methylation and proteomic characterization, we now stand ready to translate information from these technologies to the care of HCC patients, transforming the selection of systemic therapy and the selection of optimal candidates for loco-regional therapies. Results suggesting that CTNNB1-mutated HCCs are immune excluded and potentially resistant to immune checkpoint inhibitors(198200 ) but potentially susceptible to mTOR inhibitors are an early indication of the potential value of genomics in personalizing HCC therapy. These studies may also provide us with tools for better understanding the recent borderline negative results of phase 3 trials with single agent immune checkpoint inhibitors. Personalization of therapy using molecular and genomic signatures will require integration of molecular subclasses into clinical staging systems, to better guide treatment selection. Optimal treatment selection will depend on the ability to target oncogenic signaling pathways that drive tumorigenesis, tumor progression and metastasis. The development of new preclinical tumor models, including organoids, patient derived xenografts, and syngeneic models that preserve aspects of the immune response will be critical for the testing of novel agents and combinations. Ideally, integration of molecular profiling into the HCC treatment paradigm will require genomic data derived in real time from patients, either by tissue biopsy or through liquid biopsy-based access to circulating tumor DNA or other analytes. This will require a cultural change in the care of HCC patients, shifting from a state in which the diagnosis and evaluation of patients is performed non-invasively, to regular use of tissue biopsy and highly sensitive liquid biopsy assays. Development of robust, reproducible predictive biomarkers of high reliability is a key priority to facilitate this transition (Table 6). The first implementations of the biomarkerbased approaches should be within RCT, which should now routinely require tissue biopsy and liquid biopsy collection as a condition of trial enrollment. Tumor biopsy at screening for trial entry and liquid biopsy at different time points should be mandatory in clinical trials for advanced HCC to allow identification of prognostic and predictive biomarkers, guide clinical decision-making and improve patient outcomes.

\section{Novel endpoints might be adopted}

The revolution in drug development in HCC has created the need to revisit established conventions in trial design. OS is regarded as a core endpoint. Nonetheless, the realization that more than $60 \%$ of patients progressing after TACE and $50 \%$ of patients progressing after first-line systemic therapies receive effective next line therapies may compel the adoption of PFS as an acceptable primary endpoint for major trials (Table 1). In this position paper, we already are recommending PFS as co-primary endpoint for intermediate HCC trials, and for phase II-III trials assessing systemic therapies, with restrictive cut-points. Similarly, patient reported outcomes (PRO) should be pursued as a relevant endpoint in HCC trials, particularly as we enter an era of potent, but seemingly toxic, dual or triple combination therapies possibly associated with serious adverse events. It is important to recognize that currently many HCC patients reach a point in their therapeutic journey when they elect to forgo potentially life extending therapy in favor of approaches that optimize their quality of life. It is therefore critical to extend decisions about HCC trial design and endpoints to incorporate elements that reflect the importance of patient well-being. 


\section{FIGURE LEGENDS}

Figure 1. Modified BCLC staging system considering new effective therapies in advanced stages [modified and up-dated from EASL Guidelines(3)] Management of patients with HCC is guided by the Barcelona Clinic Liver Cancer (BCLC) staging system, which takes into account both tumor extent and the severity of the underlying liver disease, and defines five prognostic subgroups with respective treatments. Treatment for early stage tumors is with curative intent and options include radiofrequency ablation, hepatic resection, and liver transplantation. Patients with intermediate or advanced HCC are candidates for chemoembolization or systemic therapies, respectively. ${ }^{*}$ Patients with end-stage liver disease if Child-Pugh class $C$ should first be considered for liver transplantation. ${ }^{* *}$ Patients with preserved hepatic function Child-Pugh class $A$ with normal bilirubin and no portal hypertension are optimal candidates for hepatic resection. ¥The combination of atezolizumab plus bevacizumab is not yet approved but is set to become the new first-line treatment for advanced HCC(134)- DDLT, deceased donor liver transplantation; ECOG, Eastern Cooperative Oncology Group; LDLT, living donor liver transplantation; M1, distant metastasis; N1, lymph node metastasis; OS, overall survival.

Figure 2. Overall survival outcomes (HR, 95\% Cl) of phase III clinical trials testing molecular targeted therapies, checkpoint inhibitors and radioembolization in patients with advanced-stage hepatocellular carcinoma. Blue=positive trials for superiority. Orange=positive trials for non-inferiority. Black=negative trials for the primary end-point, Red=tested drug was significantly worse than the standard of care. Vertical red line at $\mathrm{HR}=1.08$ defines the upper boundary of $95 \%$ confidence interval accepted by FDA for a positive non-inferior study.

Figure 3. Correlation between progression-free survival and overall survival in 27 phase III trials of advanced HCC (modified from Llovet, J Hep 2019(20)) . Trial-level correlation between endpoints using criteria from the Institute for Quality and Efficiency in Health Care (IQWIG). R and R2 refers to the weighted Pearson coefficient between the HR of OS and the HR of the surrogate endpoint. Each dot represents one of the phase III clinical trials conducted on advanced HCC. Size of the dot is proportional to the total number of patients enrolled in the trial. First 21 phase III trials defined a cut-off of 0.6 for PFS to correlate with a significant OS (colored in grey)(20). Afterwards, six additional phase III trials have been reported afterwards: two positive for survival show a HR for PFS <0.6 (green color) and four negative for OS show a PFS HR> 0.6 (red color). X-and Y-axis depict the value of the HR for the surrogate and the hard endpoint, respectively. Gray shaded areas represent the upper and lower limits of the 95\% confidence intervals for the regression. $\mathrm{HCC}$, hepatocellular carcinoma; HR, hazard ratio; OS, overall survival. 


\section{TABLES}

Table 1. Recommendations for Trial design and endpoints in patients with HCC by AASLD panel of experts.

\begin{tabular}{|c|c|c|}
\hline Aim & Factor & $\begin{array}{l}\text { Considerations } \\
\text { recommendations }\end{array}$ \\
\hline \multirow[t]{3}{*}{$\begin{array}{l}\text { Select the target } \\
\text { population }\end{array}$} & BCLC stage & $\begin{array}{l}\text { Include patients according to } \\
\text { specific BCLC stage }(0-C)\end{array}$ \\
\hline & Child-Pugh classification & $\begin{array}{l}\text { Include patients in Child-Pugh } \\
\text { class A. } \\
\text { Consider ALBI grade and MELD } \\
\text { score for refinements on Child A } \\
\text { class }\end{array}$ \\
\hline & $\begin{array}{l}\text { Biomarker-based } \\
\text { enrichment }\end{array}$ & $\begin{array}{l}\text { Define rationale for using } \\
\text { biomarker and tool }\end{array}$ \\
\hline \multirow[t]{3}{*}{ Selection of endpoints } & Overall survival (OS) & $\begin{array}{l}\text { Primary endpoint for phase II and } \\
\text { III studies assessing treatments } \\
\text { in intermediate and advanced } \\
\text { HCC. }\end{array}$ \\
\hline & $\begin{array}{l}\text { Progression-free survival } \\
\text { (PFS) }\end{array}$ & $\begin{array}{l}\text { Primary endpoint for Phase II } \\
\text { studies assessing primary } \\
\text { treatments in intermediate and } \\
\text { advanced HCC. } \\
\text { Consider co-primary in phase III } \\
\text { studies in intermediate and } \\
\text { advanced HCC, with strict rules } \\
\text { for calling superiority } \\
\text { Independent centralized blinded } \\
\text { review* }\end{array}$ \\
\hline & $\begin{array}{l}\text { Time to Progression } \\
(\mathrm{TTP})\end{array}$ & $\begin{array}{l}\text { Secondary (or co-primary) } \\
\text { endpoint for Phase II studies } \\
\text { assessing primary treatments in } \\
\text { intermediate and advanced } \\
\text { HCC. } \\
\text { Independent centralized blinded } \\
\text { review* }^{*}\end{array}$ \\
\hline
\end{tabular}




\begin{tabular}{|c|c|c|}
\hline & $\begin{array}{l}\text { Recurrence-free survival } \\
\text { (RFS) }\end{array}$ & $\begin{array}{l}\text { Primary endpoint in phase II/III } \\
\text { studies assessing adjuvant } \\
\text { treatments }\end{array}$ \\
\hline & $\begin{array}{l}\text { Time to recurrence } \\
(\mathrm{TTR})\end{array}$ & $\begin{array}{l}\text { Primary endpoint }\left(2^{\text {nd }} \text { choice }\right) \text { in } \\
\text { phase II/II studies assessing } \\
\text { adjuvant treatments }\end{array}$ \\
\hline & Objective response rate & $\begin{array}{l}\text { Phase II co-primary endpoint, } \\
\text { Phase III secondary end-point }\end{array}$ \\
\hline & & $\begin{array}{l}\text { Surrogate endpoint for } \\
\text { accelerated approval }\end{array}$ \\
\hline & & $\begin{array}{lcr}\text { Independent } & \text { blinded review } \\
\text { assessing } & \text { mRECIST } & \text { for } \\
\text { interventions } & \text { at } & \text { early/ } \\
\text { intermediate } & \text { HCC. Both RECIST } \\
1.1 \text { and } & \text { mRECIST for } \\
\text { interventions at advanced stages }\end{array}$ \\
\hline & $\begin{array}{l}\text { Patient reported } \\
\text { outcomes (PRO) }\end{array}$ & $\begin{array}{l}\text { Recommended as secondary } \\
\text { endpoint in all phase III } \\
\text { investigations, particularly when } \\
\text { testing loco-regional or systemic } \\
\text { therapies }\end{array}$ \\
\hline & Composite endpoints & $\begin{array}{l}\text { OS plus PFS might be } \\
\text { considered }\end{array}$ \\
\hline Select control arm & Surveillance & Ultrasound with or without AFP \\
\hline & $\begin{array}{l}\text { Adjuvant therapy } \text { after } \\
\text { resection or } \\
\text { ablation for early } \\
\text { HCC }\end{array}$ & Placebo \\
\hline & Early stage-non surgical & $\begin{array}{l}\text { Radiofrequency ablation. Special } \\
\text { consideration for single large } \\
(>4 \mathrm{~cm} \text { tumoral diameter) when } \\
\text { standard of care is TACE as per } \\
\text { the stage migration principle. }\end{array}$ \\
\hline & $\begin{array}{l}\text { Intermediate stage } \\
\text { disease }\end{array}$ & Chemoembolization** \\
\hline & $\begin{array}{l}\text { First line treatment for } \\
\text { advanced stage disease }\end{array}$ & $\begin{array}{l}\text { Atezolizumab plus bevacizumab } \\
\text { will be considered when }\end{array}$ \\
\hline
\end{tabular}




\begin{tabular}{|c|c|c|}
\hline & & $\begin{array}{l}\text { approved. Sorafenib or lenvatinib } \\
\text { plus supportive care } \\
\text { Other treatments in Asia** }\end{array}$ \\
\hline & $\begin{array}{l}\text { Second-line treatment } \\
\text { for advanced stage } \\
\text { disease }\end{array}$ & $\begin{array}{l}\text { Regorafenib (only in patients } \\
\text { tolerant to sorafenib) or } \\
\text { cabozantinib. Ramucirumab only } \\
\text { in patients with AFP }>400 \mathrm{ng} / \mathrm{ml}\end{array}$ \\
\hline & $\begin{array}{l}\text { Third-line treatment for } \\
\text { advanced stage disease }\end{array}$ & Placebo \\
\hline \multirow[t]{4}{*}{$\begin{array}{l}\text { To stratify factors before } \\
\text { randomization }\end{array}$} & Adjuvant & $\begin{array}{l}\text { A) Geographical region } \\
\text { B) Tumor size and number } \\
\text { C) Type of curative treatment } \\
\text { D) Pathological factors of high } \\
\text { risk (size >3cm, microvascular } \\
\text { invasion, poor differentiation } \\
\text { degree and tumor satellites) }\end{array}$ \\
\hline & Intermediate-stage & $\begin{array}{l}\text { Child-Pugh class, AFP >400 ng/ } \\
\text { ml and geographical region. ALBI } \\
\text { score might be considered. } \\
\text { Selection of large tumoral burden } \\
\text { as per above } 7 \text {-up-to- } 7 \text { has been } \\
\text { proposed (adopting criteria for } \\
\text { extended indications in liver } \\
\text { transplantation), but requires } \\
\text { validation }\end{array}$ \\
\hline & First-line advanced stage & $\begin{array}{l}\text { ECOG status, MVI-EHS, AFP } \\
>400 \mathrm{ng} / \mathrm{ml} \text { and geographical } \\
\text { region, Etiology (HCV vs others } \\
\text { when testing sorafenib) }\end{array}$ \\
\hline & $\begin{array}{l}\text { Second-line advanced } \\
\text { stage }\end{array}$ & $\begin{array}{l}\text { ECOG status, MVI, EHS, } \\
\text { geographical region, AFP }>400 \\
\text { ng/ ml. Type of progression might } \\
\text { be considered }\end{array}$ \\
\hline
\end{tabular}


AFP, a-fetoprotein; BCLC, Barcelona Clinic Liver Cancer; ECOG, Eastern Cooperative Oncology Group; HCC; hepatocellular carcinoma; EHS=extrahepatic spread; MVI=macrovascular invasion; RECIST, Response Evaluation Criteria In Solid Tumours.

* $=$ not always recommended

$* *=$ Asian guidelines recommend additional treatments for

Intermediate HCC: Japan: HAIC; China: Resection; Taiwan: Resection/Y90

Advanced HCC: Japan : HAIC/Resection/TACE; China: FOLFOX4, resection/TACE; Korea: TACE 
Table 2. Expected outcomes reported in phase III trials in HCC research.

\begin{tabular}{|c|c|c|c|c|}
\hline $\begin{array}{l}\text { Expected } \\
\text { outcomes }\end{array}$ & Early & Intermediate & $\begin{array}{l}\text { Advanced } \\
\left(1^{\text {st }} \text { line }\right)^{* * *}\end{array}$ & $\begin{array}{l}\text { Advanced } \\
\left(2^{\text {nd }} \text { line }\right)\end{array}$ \\
\hline $\begin{array}{l}\text { Overall } \\
\text { survival }\end{array}$ & & $\begin{array}{lr}\text { TACE: } & 21 \\
26 \mathrm{mo}(93) \\
\mathrm{mo}(96) & (95)-33 \\
\end{array}$ & $\begin{array}{l}\text { Sorafenib: 11-14(7,11,24) } \\
\text { mo } \\
\text { Lenvatinib: } \sim 13 \mathrm{mo}(7) \\
\begin{array}{l}\text { Atezolizumab+bevacizumab } \\
* * *(134)\end{array}\end{array}$ & $\begin{array}{l}\text { Regorafenib: } \sim 11 \mathrm{mo}(8) \\
\text { Cabozantinib: } \sim 10 \mathrm{mo}(9) \\
\text { Ramucirumab (only AFP> } \\
400 \mathrm{ng} / \mathrm{ml} \text { ): } \sim 8 \mathrm{mo}(10)\end{array}$ \\
\hline PFS & $\begin{array}{l}\text { RFS*adjuvant } \\
\text { resection } \\
\text { /ablation: } 33 \mathrm{mo}\end{array}$ & TACE: $7 \mathrm{mo}(93,95)$ & $\begin{array}{l}\text { Sorafenib: } \sim 4(7) \text { months } \\
\text { Lenvatinib: } \sim 7 \text { months }(7)\end{array}$ & $\begin{array}{l}\text { Regorafenib: } \sim 3 \mathrm{mo}(8) \\
\text { Cabozantinib: } \sim 5 \mathrm{mo}(9) \\
\text { Ramucirumab (only AFP> } \\
400 \mathrm{ng} / \mathrm{ml}): \sim 3 \mathrm{mo}(10)\end{array}$ \\
\hline $\mathrm{ORR}^{*}$ & & $\begin{array}{l}\text { TACE: } \quad \sim 45-54 \% \\
(93)\end{array}$ & $\begin{array}{l}\text { Sorafenib: } \sim 10 \%(7) \\
\text { Lenvatinib: } \sim 24 \%(7) \\
\text { Atezolizumab+bevacizumab } \\
: 33 \% \text {; RECIST: } 27 \%(134)\end{array}$ & $\begin{array}{l}\text { Regorafenib: } \sim 10 \%(8) \\
\text { Cabozantinib: } \sim 4 \%(9) \\
\text { Ramucirumab (only AFP> } \\
400 \mathrm{ng} / \mathrm{ml} \text { ): } \sim 5 \%(10)\end{array}$ \\
\hline
\end{tabular}

${ }^{*}$ RFS: Recurrence free survival

** ORR as per mRECIST

***Atezolizumab+bevacizumab is expected to be fist line, while sorafenib and lenvatinib will be second line therapies, see Fig 1 
Table 3. Phases of Surveillance Test Validation in Hepatocellular Carcinoma [(adopted from Pepe et al(57))].

\begin{tabular}{|c|c|c|c|c|}
\hline Phases & $\begin{array}{ll}\text { Type } & \text { of } \\
\text { Study } & \end{array}$ & Design & Aims & Comments \\
\hline 1 & $\begin{array}{l}\text { Preclinical } \\
\text { exploratory }\end{array}$ & $\begin{array}{l}\text { Case-control from } \\
\text { biobanked } \\
\text { samples }\end{array}$ & $\begin{array}{l}\text { Promising } \quad \mathrm{HCC} \\
\text { biomarkers } \\
\text { identified }\end{array}$ & $\begin{array}{lr}\text { Avoid multiple } \\
\text { freeze-thaw for } \\
\text { blood and tissue } \\
\text { samples }\end{array}$ \\
\hline 2 & $\begin{array}{l}\text { Clinical Assay } \\
\text { and } \\
\text { Validation }\end{array}$ & $\begin{array}{l}\text { Large case- } \\
\text { controlled } \\
\text { accounting for } \\
\text { confounders }\end{array}$ & $\begin{array}{lr}\text { Biomarker } & \text { detects } \\
\text { clinically } & \\
\text { established } & \text { early } \\
\text { stage HCC } & \end{array}$ & $\begin{array}{l}\text { Appropriate sample } \\
\text { size and power } \\
\text { essential }\end{array}$ \\
\hline 3 & $\begin{array}{l}\text { Retrospective } \\
\text { longitudinal }\end{array}$ & PRoBE & $\begin{array}{l}\text { Biomarker detects } \\
\text { pre-clinical HCC }\end{array}$ & $\begin{array}{l}\text { Assess benefits } \\
\text { and harms of } \\
\text { surveillance }\end{array}$ \\
\hline 4 & $\begin{array}{l}\text { Prospective } \\
\text { screening }\end{array}$ & $\begin{array}{l}\text { Prospective } \\
\text { cirrhosis cohort }\end{array}$ & $\begin{array}{l}\text { Confirms the ability } \\
\text { of the novel } \\
\text { biomarker to detect } \\
\text { early stage disease }\end{array}$ & $\begin{array}{l}\text { Assess benefits } \\
\text { and harms }\end{array}$ \\
\hline 5 & $\begin{array}{l}\text { Cancer } \\
\text { control }\end{array}$ & $\begin{array}{l}\text { Randomized } \\
\text { study of new } \\
\text { biomarker } \\
\text { compared to US } \\
\text { and AFP }\end{array}$ & $\begin{array}{l}\text { Impact of screening } \\
\text { on reducing } \\
\text { mortality in patients } \\
\text { with cirrhosis (or } \\
\text { high-risk } \\
\text { populations) }\end{array}$ & $\begin{array}{lr}\text { Survival primary } \\
\text { endpoint; } \\
\text { secondary } \\
\text { early } \\
\text { detection, } r \text { assess } \\
\text { harms }\end{array}$ \\
\hline
\end{tabular}


Table 4. Guidelines recommendations for treatment according to levels of evidence* and strength of recommendation ${ }^{* *}$. Treatments accepted in guidelines (EASL(3) and $A A S L D(4)$ ) and level of evidence (modified from Llovet et al(119))

\begin{tabular}{|c|c|c|c|c|}
\hline Category & Treatment & $\begin{array}{l}\text { Eligibility criteria or } \\
\text { alternative approaches }\end{array}$ & $\begin{array}{l}\text { Evide } \\
\text { nce } \\
\text { level }\end{array}$ & $\begin{array}{l}\text { Recommendation } \\
\text { strength }\end{array}$ \\
\hline \multirow[t]{2}{*}{$\begin{array}{l}\text { Surgical } \\
\text { treatment }\end{array}$} & Resection & $\begin{array}{l}\text { Patient with solitary } \\
\text { tumors and well- } \\
\text { preserved liver function }\end{array}$ & $2 \mathrm{~A}$ & Strong \\
\hline & Liver transplantation & $\begin{array}{l}\text { Patients with single } \\
\text { tumors of } \leq 5 \mathrm{~cm} \text { or } \leq 3 \\
\text { nodules of } \geq 3 \mathrm{~cm} \text { (Milan } \\
\text { criteria) not suitable for } \\
\text { resection. Down staging } \\
\text { to Milan *. }\end{array}$ & $2 \mathrm{~A}$ & $\begin{array}{l}\text { Strong; } \\
\text { Moderate: down } \\
\text { staging (US), not } \\
\text { recommended in } \\
\text { Europe }\end{array}$ \\
\hline \multirow[t]{2}{*}{$\begin{array}{l}\text { Loco- } \\
\text { regional } \\
\text { treatment }\end{array}$} & Local-ablation & $\begin{array}{l}\text { Radiofrequency, BCLC } \\
0-A \text { not suitable for } \\
\text { surgery, upper limit 4- } \\
5 \mathrm{~cm} \\
\text { Radiofrequency ablation } \\
\text { (and alternatively } \\
\text { percutaneous ethanol) } \\
\text { injection for patients with } \\
\text { BCLC 0-A tumours that } \\
\text { are not suitable for } \\
\text { surgery. }\end{array}$ & $\begin{array}{l}2 \mathrm{~A} \\
2 \mathrm{~B}\end{array}$ & Strong \\
\hline & Chemoembolization & $\begin{array}{l}\text { BCLC B (multinodular } \\
\text { asymptomatic tumours } \\
\text { without vascular invasion } \\
\text { or extra-hepatic spread) }\end{array}$ & $1 \mathrm{~A}$ & Strong \\
\hline \multirow[t]{2}{*}{$\begin{array}{l}\text { Systemic } \\
\text { treatment }\end{array}$} & $\begin{array}{l}\text { Atezolizumab+ } \\
\text { bevacizumab }\end{array}$ & $\begin{array}{l}\text { Child-Pugh A } \\
\text { Advanced HCC tumours } \\
\text { (BCLC C) or BCLC B } \\
\text { progressing upon loco- } \\
\text { regional therapies }\end{array}$ & $1 \mathrm{~A}$ & $\begin{array}{lr}\begin{array}{l}\text { Strong (not yet } \\
\text { included } \\
\text { guidelines) }\end{array} & \text { in } \\
\end{array}$ \\
\hline & Sorafenib & Child-Pugh A & $1 \mathrm{~A}$ & Strong \\
\hline
\end{tabular}




\begin{tabular}{|c|c|c|c|c|}
\hline & & $\begin{array}{l}\text { Advanced HCC tumours } \\
\text { (BCLC C) or BCLC B } \\
\text { progressing upon loco- } \\
\text { regional therapies }\end{array}$ & & \\
\hline & Lenvatinib & $\begin{array}{l}\text { Child-Pugh A } \\
\text { Advanced HCC tumours } \\
\text { (BCLC C) or BCLC B } \\
\text { progressing upon loco- } \\
\text { regional therapies. No } \\
\text { Main portal vein invasion }\end{array}$ & $1 \mathrm{~A}$ & Strong \\
\hline & Regorafenib & $\begin{array}{l}\text { Child-Pugh A } \\
\text { Tolerant to sorafenib. } \\
\text { Advanced HCC } \\
\text { progressing on sorafenib }\end{array}$ & $1 \mathrm{~A}$ & Strong \\
\hline & Ramucirumab & $\begin{array}{l}\text { Child-Pugh A } \\
\text { Advanced HCC } \\
\text { progressing on sorafenib } \\
\text { AFP> } 400 \mathrm{ng} / \mathrm{dL}\end{array}$ & $1 \mathrm{~A}$ & Strong \\
\hline & Cabozantinib & $\begin{array}{l}\text { Child-Pugh A } \\
\text { Advanced HCC } \\
\text { progressing on sorafenib }\end{array}$ & $1 \mathrm{~A}$ & Strong \\
\hline $\begin{array}{l}\text { Palliative } \\
\text { care }\end{array}$ & Palliative support & $\begin{array}{l}\text { Patients with BCLC D } \\
\text { tumours should receive } \\
\text { management of pain, } \\
\text { nutrition and } \\
\text { psychological support }\end{array}$ & $2 B$ & \\
\hline \multicolumn{5}{|c|}{$\begin{array}{l}\text { Treatments under investigation or with further evidence required to be adopted in } \\
\text { guidelines }\end{array}$} \\
\hline \multirow[t]{2}{*}{$\begin{array}{l}\text { Surgical } \\
\text { treatment }\end{array}$} & \multirow[t]{2}{*}{ Resection } & $\begin{array}{l}\text { Patients with multifocal } \\
\text { small tumors }(\leq 3 \\
\text { nodules } \leq 3 \mathrm{~cm}) \text { or mild } \\
\text { portal hypertension })\end{array}$ & $3 \mathrm{~A}$ & Moderate \\
\hline & & $\begin{array}{lr}\text { Adjuvant } & \text { treatments } \\
\text { after } & \text { resection/local } \\
\text { ablation } & \end{array}$ & $1 \mathrm{D}$ & $\begin{array}{l}\text { Strongly not } \\
\text { recommended }\end{array}$ \\
\hline
\end{tabular}




\begin{tabular}{|c|c|c|c|c|}
\hline & \multirow[t]{3}{*}{ Liver transplantation } & $\begin{array}{l}\text { Up-to-seven criteria in } \\
\text { patients without } \\
\text { microvascular invasion }\end{array}$ & \multirow{2}{*}{\begin{tabular}{|l|}
$2 \mathrm{~B}$ \\
$2 \mathrm{D}$
\end{tabular}} & \multirow{2}{*}{\begin{tabular}{|l|} 
Moderate \\
Moderate \\
\end{tabular}} \\
\hline & & $\begin{array}{l}\text { Neo-adjuvant loco- } \\
\text { regional therapies if the } \\
\text { waiting list exceeds } 6 \\
\text { months }\end{array}$ & & \\
\hline & & $\begin{array}{l}\text { Living donor liver } \\
\text { transplantation } \\
\text { patients with a waiting } \\
\text { list exceeding } 6-7 \\
\text { months }\end{array}$ & $2 A$ & Moderate \\
\hline \multirow[t]{5}{*}{$\begin{array}{l}\text { Loco- } \\
\text { regional } \\
\text { treatment }\end{array}$} & 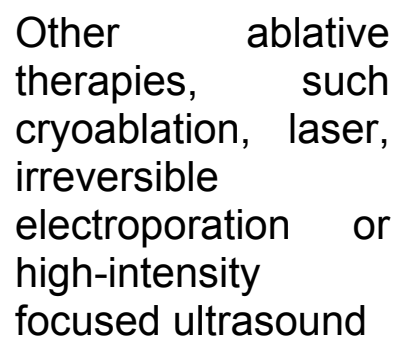 & $\begin{array}{l}\text { Patients with BCLC 0-A } \\
\text { tumours that are not } \\
\text { suitable for surgery }\end{array}$ & N/A & Not recommended \\
\hline & Chemoembolization & $\begin{array}{l}\text { Use of drug-eluting } \\
\text { beads, which has shown } \\
\text { similar response rates as } \\
\text { gelfoam-lipiodol particles } \\
\text { associated with less } \\
\text { systemic adverse events }\end{array}$ & $1 \mathrm{D}$ & Moderate \\
\hline & $\begin{array}{l}\text { Chemoembolization } \\
\text { combined } \quad \text { with } \\
\text { systemic TKis }\end{array}$ & $\begin{array}{l}\text { Multiple RCT failed to } \\
\text { show } \\
\text { outcomes }\end{array}$ & $1 \mathrm{~A}$ & Not recommended \\
\hline & $\begin{array}{l}\text { Y90- } \\
\text { Radioembolization- }\end{array}$ & $\begin{array}{l}\text { In patients at stage } \\
B C L C B \text { and in patients } \\
\text { BCLC A with a single } \\
\text { nodule larger than } 4 \mathrm{~cm} \\
\text { as an alternative to } \\
\text { resection }\end{array}$ & $1 \mathrm{D}$ & $\begin{array}{l}\text { No } \\
\text { recommendation }\end{array}$ \\
\hline & $\begin{array}{l}\text { External } \\
\text { conformal }\end{array}$ & $\begin{array}{l}\text { Single tumors at early } \\
\text { stages (BCLC A) }\end{array}$ & $3 A$ & $\begin{array}{l}\text { No } \\
\text { recommendation }\end{array}$ \\
\hline
\end{tabular}




\begin{tabular}{|c|c|c|c|c|}
\hline $\begin{array}{l}\text { Systemic } \\
\text { treatment }\end{array}$ & $\begin{array}{l}\text { Molecular targeted } \\
\text { therapies and } \\
\text { immune-based } \\
\text { therapies }\end{array}$ & $\begin{array}{l}\text { - Patients BCLC A as } \\
\text { neo-adjuvant therapies. } \\
\text { - Patients BCLC B in } \\
\text { combination with TACE, } \\
\text { Child Pugh A class, } \\
\text { ECOG } 0-1\end{array}$ & $1 \mathrm{~A}$ & $\begin{array}{l}\text { No } \\
\text { recommendation }\end{array}$ \\
\hline $\begin{array}{l}\text { Palliative } \\
\text { care }\end{array}$ & $\begin{array}{l}\text { Radiotherapy to } \\
\text { alleviate pain }\end{array}$ & $\begin{array}{l}\text { Patients with bone } \\
\text { metastasis }\end{array}$ & $3 \mathrm{~A}$ & Moderate \\
\hline \multicolumn{5}{|c|}{$\begin{array}{l}\text { AASLD, American Association for the Study of Liver Diseases; BCLC, Barcelona Clin } \\
\text { Liver Cancer Group; EASL, European Association for the Study of the Liver; HC } \\
\text { hepatocellular carcinoma. }\end{array}$} \\
\hline \multicolumn{5}{|c|}{$\begin{array}{l}{ }^{*} \text { National Cancer Institute classification: Strength of evidence: Level \#1 (RCT or meta- } \\
\text { analysis); \#2 non-randomized controlled studies; \#3 case series; Strength of end-point } \\
\text { A: survival B: cancer-specific survival; C Quality of life, and D: others. }{ }^{* *}\end{array}$} \\
\hline \multicolumn{5}{|c|}{ Table modified from EASL-EORTC guidelines(5) } \\
\hline
\end{tabular}




\begin{tabular}{|c|c|c|c|}
\hline \multicolumn{2}{|l|}{ Evaluation } & RECIST 1.1 & mRECIST \\
\hline \multirow{3}{*}{$\begin{array}{l}\text { Baseline } \\
\text { Assessment }\end{array}$} & $\begin{array}{l}\text { Target } \\
\text { lesions }\end{array}$ & $\begin{array}{l}\text { - Identify up to } 2 \text { intrahepatic } \\
\text { tumor lesions } \geq 1 \mathrm{~cm} \text {, that appear } \\
\text { suitable for accurate and repeat } \\
\text { assessments; measure their } \\
\text { longest viable tumor diameter. } \\
\text { - Identify extrahepatic tumor } \\
\text { lesions that are } \geq 1 \mathrm{~cm} \text { in longest } \\
\text { diameter and appear suitable for } \\
\text { accurate and repeat } \\
\text { assessments; measure their } \\
\text { longest overall tumor diameter. } \\
\text { - When selecting lymph nodes } \\
\text { as extrahepatic target lesions, } \\
\text { the short axis must be measured } \\
\text { and it must be } \geq 1.5 \mathrm{~cm} \text {. } \\
\text { - Overall, include a maximum of } \\
2 \text { target lesions per organ and } 5 \\
\text { target lesions in total. }\end{array}$ & $\begin{array}{l}\text { - Identify up to } 2 \text { intrahepatic tumor } \\
\text { lesions } \geq 1 \mathrm{~cm} \text {, that show typical } \\
\text { intratumoral arterial enhancement } \\
\text { and appear suitable for accurate } \\
\text { and repeat assessments; measure } \\
\text { their longest viable tumor diameter. } \\
\text { - Identify extrahepatic tumor lesions } \\
\text { (and intrahepatic lesions with } \\
\text { atypical enhancement in patients } \\
\text { without typical intrahepatic lesions) } \\
\text { that are } \geq 1 \mathrm{~cm} \text { in longest diameter } \\
\text { and appear suitable for accurate } \\
\text { and repeat assessments; measure } \\
\text { their longest overall tumor diameter. } \\
\text { - When selecting lymph nodes as } \\
\text { extrahepatic target lesions, the } \\
\text { short axis must be measured: it } \\
\text { must be } \geq 1.5 \mathrm{~cm} \text { for all lymph nodes } \\
\text { except for porta hepatis lymph } \\
\text { nodes where it is required that it is } \geq \\
2 \mathrm{~cm} \text {. }\end{array}$ \\
\hline & $\begin{array}{l}\text { Non- } \\
\text { target } \\
\text { lesions }\end{array}$ & $\begin{array}{l}\text { - Tumor lesions or sites of } \\
\text { disease that have not been } \\
\text { selected as target lesions should } \\
\text { be recorded at baseline as non- } \\
\text { target lesions. }\end{array}$ & $\begin{array}{l}\text { - Tumor lesions or sites of disease } \\
\text { that have not been selected as } \\
\text { target lesions should be recorded at } \\
\text { baseline as non-target lesions. } \\
\text { - Malignant portal vein thrombosis } \\
\text { should be considered as a non- } \\
\text { target lesions. } \\
\text { - Ascites and pleural effusions } \\
\text { should not be considered as tumor } \\
\text { lesions, unless associated with } \\
\text { unequivocal neoplastic peritoneal or } \\
\text { pleural nodules. }\end{array}$ \\
\hline & & $\begin{array}{l}\text { - Measure the longest overall } \\
\text { tumor diameter for intrahepatic }\end{array}$ & $\begin{array}{l}\text { - Measure the longest viable tumor } \\
\text { diameter of typical intrahepatic }\end{array}$ \\
\hline
\end{tabular}




\begin{tabular}{|c|c|c|c|}
\hline \multirow[t]{3}{*}{$\begin{array}{l}\text { Post- } \\
\text { Baseline } \\
\text { Assessments }\end{array}$} & $\begin{array}{l}\text { Target } \\
\text { lesions }\end{array}$ & $\begin{array}{l}\text { and non-nodal extrahepatic } \\
\text { target lesions, and the short axis } \\
\text { diameter for nodal target lesions. }\end{array}$ & $\begin{array}{l}\text { target lesions avoiding the inclusion } \\
\text { of any major intervening areas of } \\
\text { necrosis. } \\
\text { - Pay attention in distinguishing } \\
\text { areas of tumor necrosis from areas } \\
\text { of reduced arterial perfusion caused } \\
\text { by changes in local hemodynamics. } \\
\text { A change from hypervascularity to } \\
\text { hypovascularity does not represent } \\
\text { tumor necrosis. Only tumors or } \\
\text { tumor areas that show complete } \\
\text { absence of contrast enhancement } \\
\text { can be assumed to represent } \\
\text { necrotic tissue. } \\
\text { - Measure the longest overall tumor } \\
\text { diameter for atypical intrahepatic } \\
\text { target lesions and non-nodal } \\
\text { extrahepatic target lesions, and the } \\
\text { short axis diameter for nodal target } \\
\text { lesions }\end{array}$ \\
\hline & $\begin{array}{l}\text { Non- } \\
\text { target } \\
\text { lesions }\end{array}$ & $\begin{array}{l}\text { - Qualitative assessment of } \\
\text { response. }\end{array}$ & $\begin{array}{l}\text { - Qualitative assessment of } \\
\text { response, taking into account tumor } \\
\text { necrosis for typical intrahepatic non- } \\
\text { target lesions. } \\
\text { - Complete disappearance of } \\
\text { enhancement inside malignant } \\
\text { portal vein thrombus should be } \\
\text { considered equivalent to complete } \\
\text { regression }\end{array}$ \\
\hline & $\begin{array}{l}\text { New } \\
\text { lesions }\end{array}$ & $\begin{array}{l}\text { Any new lesion that has no } \\
\text { corresponding lesion on baseline } \\
\text { imaging and is unequivocally } \\
\text { malignant is considered as } \\
\text { evidence of PD. }\end{array}$ & $\begin{array}{l}\text { - By definition, a new lesion has no } \\
\text { corresponding lesion on the } \\
\text { baseline imaging. } \\
\text { - A new liver lesion } \geq 1 \mathrm{~cm} \text { that } \\
\text { shows } \\
\text { hypervascularization in the arterial } \\
\text { phase with nonperipheral washout } \\
\text { in the portal venous or the delayed } \\
\text { phase meets the criteria for } \\
\text { unequivocal new lesion and } \\
\text { declares PD. } \\
\text { - Any new liver lesion }<1 \mathrm{~cm} \text { or any } \\
\text { new liver lesion of any size that fails } \\
\text { to show the enhancement pattern } \\
\text { described above should be } \\
\text { considered as equivocal and can } \\
\text { only be diagnosed as HCC by } \\
\text { evidence of either a change in } \\
\text { enhancement pattern (when } \geq 1 \mathrm{~cm} \text { ) } \\
\text { or an interval growth } \geq 1 \mathrm{~cm} \text { in } \\
\text { subsequent scans. } \\
\text { - If an equivocal new lesion is later } \\
\text { determined to be unequivocal, the } \\
\text { timepoint of progression will be the }\end{array}$ \\
\hline
\end{tabular}




\begin{tabular}{|l|l|l|l|}
\hline & & & $\begin{array}{l}\text { timepoint that the lesion was first } \\
\text { noted as equivocal. } \\
\text { - Ascites or pleural effusion that } \\
\text { appear during treatment should not } \\
\text { be assumed to represent PD, } \\
\text { unless associated with the } \\
\text { emergence of unequivocal } \\
\text { neoplastic peritoneal or pleural } \\
\text { nodules. }\end{array}$ \\
\hline
\end{tabular}

Overall assessment of tumor response by RECIST 1.1 or mRECIST

Target lesions Non-target lesions New lesions Overall response

\begin{tabular}{llll} 
CR & CR & No & CR \\
CR & Non-CR-NonPD & No & PR \\
PR & Non-PD & No & PR \\
SD & Non-PD & No & SD \\
PD & Any & Yes or no & PD \\
Any & PD & Yes or no & PD \\
Any & Any & Yes & PD \\
\hline
\end{tabular}

CR, complete response; PR, partial response; $S D$, stable disease; PD, progressive disease. 
Table 6. Unmet needs in trial design in HCC.

1. Clinical trials:

- Evidence-based data from RCT to provide standard of care in

a) Adjuvant setting after resection/local ablation

b) Neo-adjuvant setting prior resection/liver transplantation

c) Define role of loco-regional therapies or SBRT in large single nonsurgical tumors

- Evidence-based data from RCT to improve the standard of care in

a) Combination or systemic therapies to improve chemoembolization in intermediate HCC

b) Combination (or triple) therapies for $1^{\text {st }}$ line advanced HCC

c) Molecular and immune-based therapies for patients with HCC and impaired liver function (Child-Pugh B).

- Pivotal proof-of-concept phase II trials and trial enrichment for oncogenic drivers or signaling pathways

- Systematic inclusion of cost-benefit analyses

2. Identification and validation of biomarkers:

- Develop biomarkers for early detection in surveillance programs

- Identify biomarkers predicting treatment response and primary resistance (tissue or liquid biopsy)

3. Quality of life \& patient reported outcomes:

- Incorporate tools for measuring quality of life into clinical trial design as an endpoint

- Systematic inclusion of patient reported outcomes

4. Molecular pathogenesis and drug development:

- Integrate molecular subclasses to the clinical staging system in order to better guide treatment allocation

- Target oncogene addiction loops that result from DNA amplifications and gene mutations or overexpression

- Improve models for pre-clinical testing of novel drugs 


\section{Table 7. Variables to be included in clinical trials assessing treatments for HCC patients}

Demographic

Tumor description

Staging system

Liver function

General health

\begin{abstract}
Age, sex, ethnicity
Underlying liver disease (cirrhosis, chronic hepatitis)

Etiology : define based upon $\mathrm{HCV}, \mathrm{HBV}$, alcohol, NASH-NAFLD and others.
\end{abstract}

Radiological characteristics: size, number of nodules, macroscopic vascular invasion, extrahepatic spread

Alpha-fetoprotein

Pathological characteristics in adjuvant trials: size, number, differentiation degree, satellites, micro and macroscopic vascular invasion, pTNM

BCLC staging classification

Bilirubin, aminotransferases, albumin, alkaline phosphatase, gamma-glutamyl transpeptidase, serum creatinine, serum sodium, prothrombin time, INR, platelet count

Presence of ascites or encephalopathy

Child - Pugh score

ALBI and MELD score

ECOG status, pain, constitutional syndrome

\footnotetext{
* Modified from Llovet et al, JNCI 2008(12) HCV = hepatitis C virus; HBV = hepatitis B virus; pTNM = pathological tumor-node-metastasis;

BCLC = Barcelona Clinic Liver Cancer; BUN = serum urea nitrogen; MELD: Model of EndStage Liver Disease; ECOG = Eastern Cooperative

Oncology Group.
} 


\section{REFERENCES}

1. Bray F, Ferlay J, Soerjomataram I, Siegel RL, Torre LA, Jemal A. Global cancer statistics 2018: GLOBOCAN estimates of incidence and mortality worldwide for 36 cancers in 185 countries. CA Cancer J. Clin. 2018; Available from: http://dx.doi.org/10.3322/caac.21492

2. Villanueva A. Hepatocellular Carcinoma. N. Engl. J. Med. 2019;380:1450-1462.

3. Galle PR, Forner A, Llovet JM, Mazzaferro V, Piscaglia F, Raoul J-L, et al. EASL Clinical Practice Guidelines: Management of hepatocellular carcinoma. J. Hepatol. 2018, 56(4):908-43

4. Marrero JA, Kulik LM, Sirlin CB, Zhu AX, Finn RS, Abecassis MM, et al. Diagnosis, Staging, and Management of Hepatocellular Carcinoma: 2018 Practice Guidance by the American Association for the Study of Liver Diseases. Hepatology. 2018;68:723750 .

5. Llovet JM, Ducreux M, Lencioni R, Di Bisceglie AM, Galle PR, Dufour JF, et al. EASLEORTC clinical practice guidelines: management of hepatocellular carcinoma. J. Hepatol. 2012;56:908-943.

6. Llovet JM, Montal R, Sia D, Finn RS. Molecular therapies and precision medicine for hepatocellular carcinoma. Nat. Rev. Clin. Oncol. [Internet]. 2018;Available from: http://dx.doi.org/10.1038/s41571-018-0073-4

7. Kudo M, Finn RS, Qin S, Han K-H, Ikeda K, Piscaglia F, et al. Lenvatinib versus sorafenib in first-line treatment of patients with unresectable hepatocellular carcinoma: a randomised phase 3 non-inferiority trial. Lancet. 2018;391:1163-1173.

8. Bruix J, Qin S, Merle P, Granito A, Huang Y-H, Bodoky G, et al. Regorafenib for patients with hepatocellular carcinoma who progressed on sorafenib treatment (RESORCE): a randomised, double-blind, placebo-controlled, phase 3 trial. Lancet. 2017;389:56-66.

9. Abou-Alfa GK, Meyer T, Cheng A-L, El-Khoueiry AB, Rimassa L, Ryoo B-Y, et al. Cabozantinib in Patients with Advanced and Progressing Hepatocellular Carcinoma. N. Engl. J. Med. 2018;379:54-63.

10. Zhu AX, Kang Y-K, Yen C-J, Finn RS, Galle PR, Llovet JM, et al. Ramucirumab after sorafenib in patients with advanced hepatocellular carcinoma and increased $\alpha-$ fetoprotein concentrations (REACH-2): a randomised, double-blind, placebocontrolled, phase 3 trial. Lancet Oncol. [Internet]. 2019;Available from: http://dx.doi.org/10.1016/S1470-2045(18)30937-9

11. Llovet JM, Ricci S, Mazzaferro V, Hilgard P, Gane E, Blanc J-F, et al. Sorafenib in advanced hepatocellular carcinoma. N. Engl. J. Med. 2008;359:378-390.

12. Llovet JM, Di Bisceglie AM, Bruix J, Kramer BS, Lencioni R, Zhu AX, et al. Design and endpoints of clinical trials in hepatocellular carcinoma. JNCl Journal of the National Cancer Institute. 2008;100:698-711.

13. Lee $\mathrm{DH}$, Vielemeyer $\mathrm{O}$. Analysis of overall level of evidence behind Infectious Diseases Society of America practice guidelines. Arch. Intern. Med. 2011;171:18-22. 
14. Jadad AR, Moore RA, Carroll D, Jenkinson C, Reynolds DJ, Gavaghan DJ, et al. Assessing the quality of reports of randomized clinical trials: is blinding necessary? Control. Clin. Trials. 1996;17:1-12.

15. Verhagen AP, de Vet HC, de Bie RA, Kessels AG, Boers M, Bouter LM, et al. The Delphi list: a criteria list for quality assessment of randomized clinical trials for conducting systematic reviews developed by Delphi consensus. J. Clin. Epidemiol. 1998;51:1235-1241.

16. Altman DG, Schulz KF, Moher D, Egger M, Davidoff F, Elbourne D, et al. The revised CONSORT statement for reporting randomized trials: explanation and elaboration. Ann. Intern. Med. 2001;134:663-694.

17. Piaggio G, Elbourne DR, Altman DG, Pocock SJ, Evans SJW, CONSORT Group. Reporting of noninferiority and equivalence randomized trials: an extension of the CONSORT statement. JAMA. 2006;295:1152-1160.

18. van Tulder M, Furlan A, Bombardier C, Bouter L, Editorial Board of the Cochrane Collaboration Back Review Group. Updated method guidelines for systematic reviews in the cochrane collaboration back review group. Spine. 2003;28:1290-1299.

19. Lopez PM, Villanueva A, Llovet JM. Systematic review: evidence-based management of hepatocellular carcinoma - an updated analysis of randomized controlled trials. Aliment. Pharmacol. Ther. 2006;23:1535-1547.

20. Llovet JM, Montal R, Villanueva A. Randomized trials and endpoints in advanced HCC: Role of PFS as a surrogate of survival. J. Hepatol. [Internet]. 2019;Available from: http://dx.doi.org/10.1016/j.jhep.2019.01.028

21. Lencioni R, Llovet JM. Modified RECIST (mRECIST) assessment for hepatocellular carcinoma. Semin. Liver Dis. 2010;30:52-60.

22. Cheng AL, Kang YK, Lin DY, Park JW, Kudo M, Qin S, et al. Sunitinib versus sorafenib in advanced hepatocellular cancer: results of a randomized phase III trial. J. Clin. Oncol. 2013;31:4067-4075.

23. Finn RS, Merle P, Granito A, Huang Y-H, Bodoky G, Pracht M, et al. Outcomes of sequential treatment with sorafenib followed by regorafenib for HCC: additional analyses from the phase 3 RESORCE trial. J. Hepatol. [Internet]. 2018;Available from: http://dx.doi.org/10.1016/j.jhep.2018.04.010

24. Checkmate 459 results [Internet]. Available from: https://news.bms.com/pressrelease/bmy/bristol-myers-squibb-announces-results-checkmate-459-studyevaluating-opdivo-nivol

25. Prasad V, Kim C, Burotto M, Vandross A. The Strength of Association Between Surrogate End Points and Survival in Oncology: A Systematic Review of Trial-Level Meta-analyses. JAMA Intern. Med. 2015;175:1389-1398.

26. Eisenhauer EA, Therasse P, Bogaerts J, Schwartz LH, Sargent D, Ford R, et al. New response evaluation criteria in solid tumours: revised RECIST guideline (version 1.1). European journal of cancer (Oxford, England : 1990). 2009;45:228-247.

27. Llovet JM, Lencioni R. mRECIST for HCC: Performance and novel refinements. J. 
Hepatol. 2020;72:288-306.

28. Edeline J, Boucher E, Rolland Y, Vauléon E, Pracht M, Perrin C, et al. Comparison of tumor response by Response Evaluation Criteria in Solid Tumors (RECIST) and modified RECIST in patients treated with sorafenib for hepatocellular carcinoma. Cancer. 2012;118:147-156.

29. Ronot M, Bouattour M, Wassermann J, Bruno O, Dreyer C, Larroque B, et al. Alternative Response Criteria (Choi, European Association for the Study of the Liver, and Modified Response Evaluation Criteria in Solid Tumors [RECIST]) Versus RECIST 1.1 in Patients With Advanced Hepatocellular Carcinoma Treated With Sorafenib. Oncologist. 2014;19:394-402.

30. Takada J, Hidaka H, Nakazawa T, Kondo M, Numata K, Tanaka K, et al. Modified response evaluation criteria in solid tumors is superior to response evaluation criteria in solid tumors for assessment of responses to sorafenib in patients with advanced hepatocellular carcinoma. BMC Res. Notes. 2015;8:609.

31. Lencioni R, Montal R, Torres F, Park J-W, Decaens T, Raoul J-L, et al. Objective response by $\mathrm{mRECIST}$ as a predictor and potential surrogate end-point of overall survival in advanced HCC. J. Hepatol. 2017;66:1166-1172.

32. Meyer T, Palmer DH, Cheng A-L, Hocke J, Loembé A-B, Yen C-J. mRECIST to predict survival in advanced hepatocellular carcinoma: Analysis of two randomised phase II trials comparing nintedanib vs sorafenib. Liver Int. 2017;37:1047-1055.

33. Burzykowski T, Buyse M. Surrogate threshold effect: an alternative measure for metaanalytic surrogate endpoint validation. Pharm. Stat. 2006;5:173-186.

34. Reig M, Rimola J, Torres F, Darnell A, Rodriguez-Lope C, Forner A, et al. Postprogression survival of patients with advanced hepatocellular carcinoma: Rationale for second-line trial design. HEPATOLOGY, Vol. 00, No. X, 2013 REIG ET AL. 2013;58:2023-2031.

35. Wolchok JD, Hoos A, O'Day S, Weber JS, Hamid O, Lebbé C, et al. Guidelines for the evaluation of immune therapy activity in solid tumors: immune-related response criteria. Clin. Cancer Res. 2009;15:7412-7420.

36. Seymour L, Bogaerts J, Perrone A, Ford R, Schwartz LH, Mandrekar S, et al. iRECIST: guidelines for response criteria for use in trials testing immunotherapeutics. Lancet Oncol. 2017;18:e143-e152.

37. Gyawali B, Kesselheim AS. Reinforcing the social compromise of accelerated approval. Nat. Rev. Clin. Oncol. [Internet]. 2018;Available from: http://dx.doi.org/10.1038/s41571-018-0066-3

38. El-Khoueiry AB, Sangro B, Yau T, Crocenzi TS, Kudo M, Hsu C, et al. Nivolumab in patients with advanced hepatocellular carcinoma (CheckMate 040): an open-label, non-comparative, phase $1 / 2$ dose escalation and expansion trial. Lancet [Internet]. 2017;Available from: http://dx.doi.org/10.1016/S0140-6736(17)31046-2

39. Zhu AX, Finn RS, Edeline J, Cattan S, Ogasawara S, Palmer D, et al. Pembrolizumab in patients with advanced hepatocellular carcinoma previously treated with sorafenib (KEYNOTE-224): a non-randomised, open-label phase 2 trial. Lancet Oncol. 
[Internet]. 2018;Available from: http://dx.doi.org/10.1016/S1470-2045(18)30351-6

40. Center for Drug Evaluation, Research. FDA grants accelerated approval to nivolumab and ipilimumab combinatio [Internet]. U.S. Food and Drug Administration. 2020 [cited 2020 Mar 26];Available from: http://www.fda.gov/drugs/resources-informationapproved-drugs/fda-grants-accelerated-approval-nivolumab-and-ipilimumabcombination-hepatocellular-carcinoma

41. Finn RS, Ryoo B-Y, Merle P, Kudo M, Bouattour M, Lim HY, et al. Pembrolizumab As Second-Line Therapy in Patients With Advanced Hepatocellular Carcinoma in KEYNOTE-240: A Randomized, Double-Blind, Phase III Trial. J. Clin. Oncol. 2019;JCO1901307.

42. Ellis LM, Bernstein DS, Voest EE, Berlin JD, Sargent D, Cortazar P, et al. American Society of Clinical Oncology perspective: Raising the bar for clinical trials by defining clinically meaningful outcomes. J. Clin. Oncol. 2014;32:1277-1280.

43. Nault J-C, Cheng A-L, Sangro B, Llovet JM. Milestones in the pathogenesis and management of primary liver cancer. J. Hepatol. 2020;72:209-214.

44. Singal AG, Lampertico $P$, Nahon $P$. Epidemiology and surveillance for hepatocellular carcinoma: New trends. J. Hepatol. 2020;72:250-261.

45. Tzartzeva K, Obi J, Rich NE, Parikh ND, Marrero JA, Yopp A, et al. Surveillance Imaging and Alpha Fetoprotein for Early Detection of Hepatocellular Carcinoma in Patients With Cirrhosis: A Meta-analysis. Gastroenterology. 2018;154:1706-1718.e1.

46. Atiq O, Tiro J, Yopp AC, Muffler A, Marrero JA, Parikh ND, et al. An assessment of benefits and harms of hepatocellular carcinoma surveillance in patients with cirrhosis. Hepatology. 2017;65:1196-1205.

47. Park J-W, Chen M, Colombo M, Roberts LR, Schwartz M, Chen P-J, et al. Global patterns of hepatocellular carcinoma management from diagnosis to death: the BRIDGE Study. Liver Int. 2015;35:2155-2166.

48. Totoki Y, Tatsuno K, Covington KR, Ueda H, Creighton CJ, Kato M, et al. Transancestry mutational landscape of hepatocellular carcinoma genomes. Nat. Genet. 2014;46:1267-1273.

49. Schulze K, Imbeaud S, Letouzé E, Alexandrov LB, Calderaro J, Rebouissou S, et al. Exome sequencing of hepatocellular carcinomas identifies new mutational signatures and potential therapeutic targets. Nat. Genet. 2015;47:505-511.

50. Ally A, Balasundaram M, Carlsen R, Chuah E, Clarke A, Dhalla N, et al. Comprehensive and Integrative Genomic Characterization of Hepatocellular Carcinoma. Cell. 2017;169:1327-1341.e23.

51. Gao Q, Zhu H, Dong L, Shi W, Chen R, Song Z, et al. Integrated Proteogenomic Characterization of HBV-Related Hepatocellular Carcinoma. Cell. 2019;179:561577.e22.

52. Johnson PJ, Pirrie SJ, Cox TF, Berhane S, Teng M, Palmer D, et al. The detection of hepatocellular carcinoma using a prospectively developed and validated model based on serological biomarkers. Cancer Epidemiol. Biomarkers Prev. 2014;23:144-153. 
53. Wang $M$, Sanda $M$, Comunale $M A$, Herrera $H$, Swindell $C$, Kono $Y$, et al. Changes in the Glycosylation of Kininogen and the Development of a Kininogen-Based Algorithm for the Early Detection of HCC. Cancer Epidemiol. Biomarkers Prev. 2017;26:795803.

54. von Felden J, Craig AJ, Villanueva A. Role of circulating tumor DNA to help decisionmaking in hepatocellular carcinoma. Oncoscience. 2018;5:209-211.

55. Kisiel JB, Dukek BA, Kanipakam RVSR, Ghoz HM, Yab TC, Berger CK, et al. Hepatocellular Carcinoma Detection by Plasma Methylated DNA: Discovery, Phase I Pilot, and Phase II Clinical Validation. Hepatology [Internet]. 2018;Available from: http://dx.doi.org/10.1002/hep.30244

56. Khatri G, Pedrosa I, Ananthakrishnan L, de Leon AD, Fetzer DT, Leyendecker J, et al. Abbreviated-protocol screening MRI vs. complete-protocol diagnostic MRI for detection of hepatocellular carcinoma in patients with cirrhosis: An equivalence study using LI-RADS v2018. J. Magn. Reson. Imaging [Internet]. 2019;Available from: http://dx.doi.org/10.1002/jmri.26835

57. Pepe MS, Etzioni R, Feng Z, Potter JD, Thompson ML, Thornquist M, et al. Phases of biomarker development for early detection of cancer. J. Natl. Cancer Inst. 2001;93:1054-1061.

58. Pepe MS, Feng Z, Janes H, Bossuyt PM, Potter JD. Pivotal evaluation of the accuracy of a biomarker used for classification or prediction: standards for study design. J. Natl. Cancer Inst. 2008;100:1432-1438.

59. Ioannou GN, Green P, Lowy E, Mun EJ, Berry K. Differences in hepatocellular carcinoma risk, predictors and trends over time according to etiology of cirrhosis. PLoS One. 2018;13:e0204412.

60. Kanwal F, Kramer JR, Mapakshi S, Natarajan Y, Chayanupatkul M, Richardson PA, et al. Risk of Hepatocellular Cancer in Patients With Non-Alcoholic Fatty Liver Disease. Gastroenterology. 2018;155:1828-1837.e2.

61. Kanwal F, Singal AG. Surveillance for Hepatocellular Carcinoma: Current Best Practice and Future Direction. Gastroenterology [Internet]. 2019;Available from: http://dx.doi.org/10.1053/j.gastro.2019.02.049

62. Yang H-I, Yeh M-L, Wong GL, Peng C-Y, Chen C-H, Trinh HN, et al. REAL-B (Realworld Effectiveness from the Asia Pacific Rim Liver Consortium for HBV) Risk Score for the Prediction of Hepatocellular Carcinoma in Chronic Hepatitis B Patients Treated with Oral Antiviral Therapy. J. Infect. Dis. [Internet]. 2019;Available from: http://dx.doi.org/10.1093/infdis/jiz477

63. Office of the Commissioner. Biomarkers at FDA [Internet]. U.S. Food and Drug Administration. 2019 [cited 2019 Nov 15];Available from: http://www.fda.gov/scienceresearch/about-science-research-fda/biomarkers-fda

64. Goodsaid FM. The Labyrinth of Product Development and Regulatory Approvals in Liquid Biopsy Diagnostics. Clin. Transl. Sci. 2019;12:431-439.

65. Simon TG, Ma Y, Ludvigsson JF, Chong DQ, Giovannucci EL, Fuchs CS, et al. Association Between Aspirin Use and Risk of Hepatocellular Carcinoma. JAMA 
Oncol. 2018;4:1683-1690.

66. Malehmir M, Pfister D, Gallage S, Szydlowska M, Inverso D, Kotsiliti E, et al. Platelet GPIba is a mediator and potential interventional target for NASH and subsequent liver cancer. Nat. Med. [Internet]. 2019;Available from: http://dx.doi.org/10.1038/s41591019-0379-5

67. Simon TG, Duberg A-S, Aleman S, Hagstrom H, Nguyen LH, Khalili H, et al. Lipophilic Statins and Risk for Hepatocellular Carcinoma and Death in Patients With Chronic Viral Hepatitis: Results From a Nationwide Swedish Population. Ann. Intern. Med. 2019;171:318-327.

68. Tseng $\mathrm{C}-\mathrm{H}$. Metformin and risk of hepatocellular carcinoma in patients with type 2 diabetes. Liver Int. 2018;38:2018-2027.

69. Heimbach JK, Kulik LM, Finn RS, Sirlin CB, Abecassis MM, Roberts LR, et al. AASLD guidelines for the treatment of hepatocellular carcinoma. Hepatology. 2018;67:358380.

70. Roayaie S, Jibara G, Tabrizian P, Park J-W, Yang J, Yan L, et al. The role of hepatic resection in the treatment of hepatocellular cancer. Hepatology. 2015;62:440-451.

71. Roayaie S, Obeidat K, Sposito C, Mariani L, Bhoori S, Pellegrinelli A, et al. Resection of hepatocellular cancer $\leq 2 \mathrm{~cm}$ : results from two Western centers. Hepatology. 2013;57:1426-1435.

72. Muto Y, Moriwaki H, Ninomiya M, Adachi S, Saito A, Takasaki KT, et al. Prevention of second primary tumors by an acyclic retinoid, polyprenoic acid, in patients with hepatocellular carcinoma. Hepatoma Prevention Study Group. N. Engl. J. Med. 1996;334:1561-1567.

73. Okita K, Izumi N, Matsui O, Tanaka K, Kaneko S, Moriwaki H, et al. Peretinoin after curative therapy of hepatitis C-related hepatocellular carcinoma: a randomized double-blind placebo-controlled study. J. Gastroenterol. 2015;50:191-202.

74. Takayama T, Sekine T, Makuuchi M, Yamasaki S, Kosuge T, Yamamoto J, et al. Adoptive immunotherapy to lower postsurgical recurrence rates of hepatocellular carcinoma: a randomised trial. Lancet. 2000;356:802-807.

75. Lau WY, Lai ECH, Leung TWT, Yu SCH. Adjuvant intra-arterial iodine-131-labeled lipiodol for resectable hepatocellular carcinoma: a prospective randomized trialupdate on 5-year and 10-year survival. Ann. Surg. 2008;247:43-48.

76. Bruix J, Takayama T, Mazzaferro V, Chau GY, Yang J, Kudo M, et al. Adjuvant sorafenib for hepatocellular carcinoma after resection or ablation (STORM): a phase 3, randomised, double-blind, placebo-controlled trial. Lancet Oncol. 2015;16:13441354.

77. Bott MJ, Yang SC, Park BJ, Adusumilli PS, Rusch VW, Isbell JM, et al. Initial results of pulmonary resection after neoadjuvant nivolumab in patients with resectable nonsmall cell lung cancer. J. Thorac. Cardiovasc. Surg. 2019;158:269-276.

78. Mazzaferro V, Regalia E, Doci R, Andreola S, Pulvirenti A, Bozzetti F, et al. Liver transplantation for the treatment of small hepatocellular carcinomas in patients with 
cirrhosis. N. Engl. J. Med. 1996;334:693-699.

79. Yao FY, Mehta N, Flemming J, Dodge J, Hameed B, Fix O, et al. Downstaging of hepatocellular cancer before liver transplant: long-term outcome compared to tumors within Milan criteria. Hepatology. 2015;61:1968-1977.

80. Sposito C, Mariani L, Germini A, Flores Reyes M, Bongini M, Grossi G, et al. Comparative efficacy of sorafenib versus best supportive care in recurrent hepatocellular carcinoma after liver transplantation: a case-control study. J. Hepatol. 2013;59:59-66.

81. Morales RE, Shoushtari AN, Walsh MM, Grewal P, Lipson EJ, Carvajal RD. Safety and efficacy of ipilimumab to treat advanced melanoma in the setting of liver transplantation. J Immunother Cancer. 2015;3:22.

82. Breen DJ, Lencioni R. Image-guided ablation of primary liver and renal tumours. Nat. Rev. Clin. Oncol. 2015;12:175-186.

83. Cho YK, Kim JK, Kim MY, Rhim H, Han JK. Systematic review of randomized trials for hepatocellular carcinoma treated with percutaneous ablation therapies. Hepatology. 2009;49:453-459.

84. Glassberg MB, Ghosh S, Clymer JW, Qadeer RA, Ferko NC, Sadeghirad B, et al. Microwave ablation compared with radiofrequency ablation for treatment of hepatocellular carcinoma and liver metastases: a systematic review and metaanalysis. Onco. Targets. Ther. 2019;12:6407-6438.

85. Nault J-C, Sutter O, Nahon P, Ganne-Carrié N, Séror O. Percutaneous treatment of hepatocellular carcinoma: State of the art and innovations. J. Hepatol. 2018;68:783797.

86. Llovet JM, Real MI, Montaña X, Planas R, Coll S, Aponte J, et al. Arterial embolisation or chemoembolisation versus symptomatic treatment in patients with unresectable hepatocellular carcinoma: a randomised controlled trial. Lancet. 2002;359:1734-1739.

87. Lo C-M, Ngan H, Tso W-K, Liu C-L, Lam C-M, Poon RT-P, et al. Randomized controlled trial of transarterial lipiodol chemoembolization for unresectable hepatocellular carcinoma. Hepatology. 2002;35:1164-1171.

88. Llovet JM, Bruix J. Systematic review of randomized trials for unresectable hepatocellular carcinoma: Chemoembolization improves survival. Hepatology. 2003;37:429-442.

89. Salem R, Mazzaferro V, Sangro B. Yttrium 90 radioembolization for the treatment of hepatocellular carcinoma: biological lessons, current challenges, and clinical perspectives. Hepatology. 2013;58:2188-2197.

90. Bolondi L, Burroughs A, Dufour J-F, Galle PR, Mazzaferro V, Piscaglia F, et al. Heterogeneity of patients with intermediate (BCLC B) Hepatocellular Carcinoma: proposal for a subclassification to facilitate treatment decisions. Semin. Liver Dis. 2012;32:348-359.

91. Kudo M, Arizumi T, Ueshima K, Sakurai T, Kitano M, Nishida N. Subclassification of 
BCLC B Stage Hepatocellular Carcinoma and Treatment Strategies: Proposal of Modified Bolondi's Subclassification (Kinki Criteria). Dig. Dis. 2015;33:751-758.

92. Forner A, Gilabert M, Bruix J, Raoul JL. Treatment of intermediate-stage hepatocellular carcinoma. Nat. Rev. Clin. Oncol. 2014;11:525-535.

93. Meyer T, Fox R, Ma YT, Ross PJ, James MW, Sturgess R, et al. Sorafenib in combination with transarterial chemoembolisation in patients with unresectable hepatocellular carcinoma (TACE 2): a randomised placebo-controlled, double-blind, phase 3 trial. Lancet Gastroenterol Hepatol. 2017;2:565-575.

94. Lencioni R, Llovet JM, Han G, Tak WY, Yang J, Guglielmi A, et al. Sorafenib or placebo plus TACE with doxorubicin-eluting beads for intermediate stage HCC: The SPACE trial. J. Hepatol. 2016;64:1090-1098.

95. Kudo M, Han G, Finn RS, Poon RT, Blanc JF, Yan L, et al. Brivanib as adjuvant therapy to transarterial chemoembolization in patients with hepatocellular carcinoma: A randomized phase III trial. Hepatology. 2014;60:1697-1707.

96. Kudo M, Cheng A-L, Park J-W, Park JH, Liang P-C, Hidaka H, et al. Orantinib versus placebo combined with transcatheter arterial chemoembolisation in patients with unresectable hepatocellular carcinoma (ORIENTAL): a randomised, double-blind, placebo-controlled, multicentre, phase 3 study. Lancet Gastroenterol Hepatol. 2018;3:37-46.

97. Johnson PJ, Berhane S, Kagebayashi C, Satomura S, Teng M, Reeves HL, et al. Assessment of liver function in patients with hepatocellular carcinoma: a new evidence-based approach-the ALBI grade. J. Clin. Oncol. 2015;33:550-558.

98. Edeline J, Blanc J-F, Johnson P, Campillo-Gimenez B, Ross P, Ma YT, et al. A multicentre comparison between Child Pugh and Albumin-Bilirubin scores in patients treated with sorafenib for Hepatocellular Carcinoma. Liver Int. 2016;36:1821-1828.

99. Kadalayil L, Benini R, Pallan L, O’Beirne J, Marelli L, Yu D, et al. A simple prognostic scoring system for patients receiving transarterial embolisation for hepatocellular cancer. Ann. Oncol. 2013;24:2565-2570.

100. Waked I, Berhane S, Toyoda H, Chan SL, Stern N, Palmer D, et al. Transarterial chemo-embolisation of hepatocellular carcinoma: impact of liver function and vascular invasion. Br. J. Cancer. 2017;116:448-454.

101. Lencioni R, de Baere T, Soulen MC, Rilling WS, Geschwind J-FH. Lipiodol transarterial chemoembolization for hepatocellular carcinoma: A systematic review of efficacy and safety data. Hepatology. 2016;64:106-116.

102. Meyer T, Kirkwood A, Roughton M, Beare S, Tsochatzis E, Yu D, et al. A randomised phase II/III trial of 3-weekly cisplatin-based sequential transarterial chemoembolisation vs embolisation alone for hepatocellular carcinoma. Br. J. Cancer. 2013;108:1252-1259.

103. Lammer J, Malagari K, Vogl T, Pilleul F, Denys A, Watkinson A, et al. Prospective randomized study of doxorubicin-eluting-bead embolization in the treatment of hepatocellular carcinoma: results of the PRECISION V study. Cardiovasc. Intervent. Radiol. 2010;33:41-52. 
104. de Baere T, Arai Y, Lencioni R, Geschwind J-F, Rilling W, Salem R, et al. Treatment of Liver Tumors with Lipiodol TACE: Technical Recommendations from Experts Opinion. Cardiovasc. Intervent. Radiol. 2016;39:334-343.

105. Gillmore R, Stuart S, Kirkwood A, Hameeduddin A, Woodward N, Burroughs AK, et al. EASL and mRECIST responses are independent prognostic factors for survival in hepatocellular cancer patients treated with transarterial embolization. J. Hepatol. 2011;55:1309-1316.

106. Vincenzi B, Di Maio M, Silletta M, D’Onofrio L, Spoto C, Piccirillo MC, et al. Prognostic Relevance of Objective Response According to EASL Criteria and mRECIST Criteria in Hepatocellular Carcinoma Patients Treated with Loco-Regional Therapies: A Literature-Based Meta-Analysis. PLoS One. 2015;10:e0133488.

107. Kudo M. Proposal of Primary Endpoints for TACE Combination Trials with Systemic Therapy: Lessons Learned from 5 Negative Trials and the Positive TACTICS Trial. Liver Cancer. 2018;7:225-234.

108. Kudo M, Ueshima K, Ikeda M, Torimura T, Tanabe N, Aikata H, et al. Randomised, multicentre prospective trial of transarterial chemoembolisation (TACE) plus sorafenib as compared with TACE alone in patients with hepatocellular carcinoma: TACTICS trial. Gut [Internet]. 2019;Available from: http://dx.doi.org/10.1136/gutjnl-2019-318934

109. Kudo M, Kubo S, Takayasu K, Sakamoto M, Tanaka M, Ikai I, et al. Response Evaluation Criteria in Cancer of the Liver (RECICL) proposed by the Liver Cancer Study Group of Japan (2009 Revised Version). Hepatol. Res. 2010;40:686-692.

110. Kudo M. A New Treatment Option for Intermediate-Stage Hepatocellular Carcinoma with High Tumor Burden: Initial Lenvatinib Therapy with Subsequent Selective TACE. Liver Cancer. 2019;8:299-311.

111. Therasse P, Arbuck SG, Eisenhauer EA, Wanders J, Kaplan RS, Rubinstein L, et al. New guidelines to evaluate the response to treatment in solid tumors. European Organization for Research and Treatment of Cancer, National Cancer Institute of the United States, National Cancer Institute of Canada. Journal of the National Cancer Institute. 2000;92:205-216.

112. Lencioni R. New data supporting modified RECIST (mRECIST) for Hepatocellular Carcinoma. Clin. Cancer Res. 2013;19:1312-1314.

113. Nishino M, Giobbie-Hurder A, Gargano M, Suda M, Ramaiya NH, Hodi FS. Developing a common language for tumor response to immunotherapy: immunerelated response criteria using unidimensional measurements. Clin. Cancer Res. 2013;19:3936-3943.

114. Bohnsack O, Hoos A, Ludajic K. Adaptation and modification of the immune related response criteria (IRRC): IrRECIST. J. Clin. Orthod. 2014;32:e22121-e22121.

115. Hodi FS, Ballinger M, Lyons B, Soria J-C, Nishino M, Tabernero J, et al. ImmuneModified Response Evaluation Criteria In Solid Tumors (imRECIST): Refining Guidelines to Assess the Clinical Benefit of Cancer Immunotherapy. J. Clin. Oncol. 2018;36:850-858.

116. Ikeda M, Sung MW, Kudo M, Kobayashi M, Baron AD, Finn RS, et al. A phase 1b 
trial of lenvatinib (LEN) plus pembrolizumab (PEM) in patients (pts) with unresectable hepatocellular carcinoma (uHCC). J. Clin. Oncol. 2018;36:4076-4076.

117. Moehler M, Heo J, Lee HC, Tak WY, Chao Y, Paik SW, et al. Vaccinia-based oncolytic immunotherapy Pexastimogene Devacirepvec in patients with advanced hepatocellular carcinoma after sorafenib failure: a randomized multicenter Phase IIb trial (TRAVERSE). Oncoimmunology. 2019;8:1615817.

118. Heo J, Reid T, Ruo L, Breitbach CJ, Rose S, Bloomston M, et al. Randomized dose-finding clinical trial of oncolytic immunotherapeutic vaccinia JX-594 in liver cancer. Nat. Med. 2013;1-10.

119. Llovet JM, Zucman-Rossi J, Pikarsky E, Sangro B, Schwartz M, Sherman M, et al. Hepatocellular carcinoma. Nature Reviews Disease Primers. 2016;2:16018-16023.

120. Bruix J, Sherman M, American Association for the Study of Liver Diseases. Management of hepatocellular carcinoma: an update. Hepatology. 2011;53:10201022.

121. Qin S, Bai Y, Lim HY, Thongprasert S, Chao Y, Fan J, et al. Randomized, multicenter, open-label study of oxaliplatin plus fluorouracil/leucovorin versus doxorubicin as palliative chemotherapy in patients with advanced hepatocellular carcinoma from Asia. J. Clin. Oncol. 2013;31:3501-3508.

122. Abou-Alfa GK, Niedzwieski D, Knox JJ, Kaubisch A, Posey J, Tan BR, et al. Phase III randomized study of sorafenib plus doxorubicin versus sorafenib in patients with advanced hepatocellular carcinoma (HCC): CALGB 80802 (Alliance). J. Clin. Orthod. 2016;34:192-192.

123. Kudo M, Ueshima K, Yokosuka O, Ogasawara S, Obi S, Izumi N, et al. Sorafenib plus low-dose cisplatin and fluorouracil hepatic arterial infusion chemotherapy versus sorafenib alone in patients with advanced hepatocellular carcinoma (SILIUS): a randomised, open label, phase 3 trial. Lancet Gastroenterol Hepatol. 2018;3:424432.

124. Yeo W, Mok TS, Zee B, Leung TWT, Lai PBS, Lau WY, et al. A randomized phase III study of doxorubicin versus cisplatin/interferon alpha-2b/doxorubicin/fluorouracil (PIAF) combination chemotherapy for unresectable hepatocellular carcinoma. J. Natl. Cancer Inst. 2005;97:1532-1538.

125. Chow PK h., Tai B-C, Tan C-K, Machin D, Win KM, Johnson PJ, et al. High-dose tamoxifen in the treatment of inoperable hepatocellular carcinoma: A multicenter randomized controlled trial. Hepatology. 2002;36:1221-1226.

126. Johnson PJ, Qin S, Park J-W, Poon RTP, Raoul J-L, Philip PA, et al. Brivanib versus sorafenib as first-line therapy in patients with unresectable, advanced hepatocellular carcinoma: results from the randomized phase III BRISK-FL study. J. Clin. Oncol. 2013;31:3517-3524.

127. Llovet JM, Decaens T, Raoul JL, Boucher E, Kudo M, Chang C, et al. Brivanib in patients with advanced hepatocellular carcinoma who were intolerant to sorafenib or for whom sorafenib failed: results from the randomized phase III BRISK-PS study. J. Clin. Oncol. 2013;31:3509-3516. 
128. Cainap C, Qin S, Huang W-T, Chung IJ, Pan H, Cheng Y, et al. Linifanib versus Sorafenib in patients with advanced hepatocellular carcinoma: results of a randomized phase III trial. J. Clin. Oncol. 2015;33:172-179.

129. Zhu AX, Rosmorduc O, Evans TRJ, Ross PJ, Santoro A, Carrilho FJ, et al. SEARCH: a phase III, randomized, double-blind, placebo-controlled trial of sorafenib plus erlotinib in patients with advanced hepatocellular carcinoma. J. Clin. Oncol. 2015;33:559-566.

130. Zhu AX, Kudo M, Assenat E, Cattan S, Kang Y-K, Lim HY, et al. Effect of everolimus on survival in advanced hepatocellular carcinoma after failure of sorafenib: the EVOLVE-1 randomized clinical trial. JAMA. 2014;312:57-67.

131. Rimassa L, Assenat E, Peck-Radosavljevic M, Pracht M, Zagonel V, Mathurin P, et al. Tivantinib for second-line treatment of MET-high, advanced hepatocellular carcinoma (METIV-HCC): a final analysis of a phase 3, randomised, placebocontrolled study. Lancet Oncol. [Internet]. 2018 [cited 2018 Apr 4];Available from: http://dx.doi.org/10.1016/S1470-2045(18)30146-3

132. Merle P, Blanc J-F, Phelip J-M, Pelletier G, Bronowicki J-P, Touchefeu Y, et al. Doxorubicin-loaded nanoparticles for patients with advanced hepatocellular carcinoma after sorafenib treatment failure (RELIVE): a phase 3 randomised controlled trial. Lancet Gastroenterol Hepatol. 2019;4:454-465.

133. Abou-Alfa GK, Qin S, Ryoo B-Y, Lu S-N, Yen C-J, Feng Y-H, et al. Phase III randomized study of second line ADI-PEG 20 plus best supportive care versus placebo plus best supportive care in patients with advanced hepatocellular carcinoma. Ann. Oncol. 2018;29:1402-1408.

134. Roche's Tecentriq in combination with Avastin increased overall survival and progression-free survival in people with unresectable hepatocellular carcinoma [Internet]. [cited 2019 Nov 4];Available from:

https://www.roche.com/media/releases/med-cor-2019-10-21.htm

135. Zhu AX, Park JO, Ryoo B-Y, Yen C-J, Poon R, Pastorelli D, et al. Ramucirumab versus placebo as second-line treatment in patients with advanced hepatocellular carcinoma following first-line therapy with sorafenib (REACH): a randomised, doubleblind, multicentre, phase 3 trial. Lancet Oncol. 2015;16:859-870.

136. Llovet JM, Pena CEA, Lathia CD, Shan M, Meinhardt G, Bruix J, et al. Plasma Biomarkers as Predictors of Outcome in Patients with Advanced Hepatocellular Carcinoma. Clin. Cancer Res. 2012;18:2290-2300.

137. Vilgrain V, Pereira H, Assenat E, Guiu B, llonca AD, Pageaux G-P, et al. Efficacy and safety of selective internal radiotherapy with yttrium-90 resin microspheres compared with sorafenib in locally advanced and inoperable hepatocellular carcinoma (SARAH): an open-label randomised controlled phase 3 trial. Lancet Oncol. [Internet]. 2017;Available from: http://dx.doi.org/10.1016/S1470-2045(17)30683-6

138. Chow PKH, Gandhi M, Tan S-B, Khin MW, Khasbazar A, Ong J, et al. SIRveNIB: Selective Internal Radiation Therapy Versus Sorafenib in Asia-Pacific Patients With Hepatocellular Carcinoma. J. Clin. Oncol. 2018;36:1913-1921.

139. Ricke J, Sangro B, Amthauer H, Bargellini I, Bartenstein P, De Toni E, et al. The 
impact of combining Selective Internal Radiation Therapy (SIRT) with Sorafenib on overall survival in patients with advanced hepatocellular carcinoma: The Soramic trial palliative cohort. J. Hepatol. 2018;68:S102.

140. Cheng A-L, Kang Y-K, Chen Z, Tsao C-J, Qin S, Kim JS, et al. Efficacy and safety of sorafenib in patients in the Asia-Pacific region with advanced hepatocellular carcinoma: a phase III randomised, double-blind, placebo-controlled trial. Lancet Oncol. 2009;10:25-34.

141. He M, Li Q, Zou R, Shen J, Fang W, Tan G, et al. Sorafenib Plus Hepatic Arterial Infusion of Oxaliplatin, Fluorouracil, and Leucovorin vs Sorafenib Alone for Hepatocellular Carcinoma With Portal Vein Invasion: A Randomized Clinical Trial. JAMA Oncol [Internet]. 2019;Available from:

http://dx.doi.org/10.1001/jamaoncol.2019.0250

142. Jouve J-L, Lecomte T, Bouché O, Barbier E, Khemissa Akouz F, Riachi G, et al. Pravastatin combination with sorafenib does not improve survival in advanced hepatocellular carcinoma. J. Hepatol. 2019;71:516-522.

143. Abou-Alfa GK, Shi Q, Knox JJ, Kaubisch A, Niedzwiecki D, Posey J, et al. Assessment of Treatment With Sorafenib Plus Doxorubicin vs Sorafenib Alone in Patients With Advanced Hepatocellular Carcinoma: Phase 3 CALGB 80802 Randomized Clinical Trial. JAMA Oncol [Internet]. 2019;Available from: http://dx.doi.org/10.1001/jamaoncol.2019.2792

144. Torre LA, Bray F, Siegel RL, Ferlay J, Lortet-Tieulent J, Jemal A. Global cancer statistics, 2012. CA Cancer J. Clin. 2015;65:87-108.

145. Gan HK, You B, Pond GR, Chen EX. Assumptions of expected benefits in randomized phase III trials evaluating systemic treatments for cancer. J. Natl. Cancer Inst. 2012;104:590-598.

146. Oxnard GR, Wilcox KH, Gonen M, Polotsky M, Hirsch BR, Schwartz LH. Response Rate as a Regulatory End Point in Single-Arm Studies of Advanced Solid Tumors. JAMA Oncol. 2016;2:772-779.

147. Sangro B, Gomez-Martin C, de la Mata M, Iñarrairaegui M, Garralda E, Barrera P, et al. A clinical trial of CTLA-4 blockade with tremelimumab in patients with hepatocellular carcinoma and chronic hepatitis C. J. Hepatol. 2013;59:81-88.

148. Qin SK, Ren ZG, Meng ZQ, Chen ZD, Chai XL, Xiong JP, et al. LBA27A randomized multicentered phase II study to evaluate SHR-1210 (PD-1 antibody) in subjects with advanced hepatocellular carcinoma (HCC) who failed or intolerable to prior systemic treatment. Ann. Oncol. [Internet]. 2018 [cited 2019 Sep 23];29. Available from: https://academic.oup.com/annonc/article/29/suppl_8/mdy424.029/5141688

149. Finn RS, Ryoo B-Y, Merle P, Kudo M, Bouattour M, Lim H-Y, et al. Results of KEYNOTE-240: phase 3 study of pembrolizumab (Pembro) vs best supportive care (BSC) for second line therapy in advanced hepatocellular carcinoma (HCC). J. Clin. Orthod. 2019;37:4004-4004.

150. Duffy AG, Ulahannan SV, Makorova-Rusher O, Rahma O, Wedemeyer H, Pratt D, et al. Tremelimumab in combination with ablation in patients with advanced 
hepatocellular carcinoma. J. Hepatol. 2017;66:545-551.

151. Yau T, Kang Y-K, Kim T-Y, El-Khoueiry AB, Santoro A, Sangro B, et al. Nivolumab (NIVO) + ipilimumab (IPI) combination therapy in patients (pts) with advanced hepatocellular carcinoma (aHCC): Results from CheckMate 040. J. Clin. Orthod. 2019;37:4012-4012.

152. Greten TF, Lai CW, Li G, Staveley-O'Carroll KF. Targeted and Immune-Based Therapies for Hepatocellular Carcinoma. Gastroenterology. 2019;156:510-524.

153. Greten TF, Sangro B. Targets for immunotherapy of liver cancer. J. Hepatol. [Internet]. 2017;Available from: http://dx.doi.org/10.1016/j.jhep.2017.09.007

154. Anagnostou V, Yarchoan M, Hansen AR, Wang H, Verde F, Sharon E, et al. Immuno-oncology Trial Endpoints: Capturing Clinically Meaningful Activity. Clin. Cancer Res. 2017;23:4959-4969.

155. Ritchie G, Gasper H, Man J, Lord S, Marschner I, Friedlander M, et al. Defining the Most Appropriate Primary End Point in Phase 2 Trials of Immune Checkpoint Inhibitors for Advanced Solid Cancers: A Systematic Review and Meta-analysis. JAMA Oncol. 2018;4:522-528.

156. Simon RM, Paik S, Hayes DF. Use of Archived Specimens in Evaluation of Prognostic and Predictive Biomarkers. JNCl Journal of the National Cancer Institute. 2009;101:1446-1452.

157. Mandrekar SJ, Sargent DJ. Clinical trial designs for predictive biomarker validation: theoretical considerations and practical challenges. J. Clin. Oncol. 2009;27:40274034.

158. Mandrekar SJ, Sargent DJ. Genomic advances and their impact on clinical trial design. Genome Med. 2009;1:69.

159. Renfro LA, An M-W, Mandrekar SJ. Precision oncology: A new era of cancer clinical trials. Cancer Lett. 2017;387:121-126.

160. Biomarker defintion $\mathrm{NCl}$ [Internet]. Available from: https://www.cancer.gov/publications/dictionaries/cancer-terms/def/biomarker

161. Rebouissou S, La Bella T, Rekik S, Imbeaud S, Calatayud A-L, Rohr-Udilova N, et al. Proliferation Markers Are Associated with MET Expression in Hepatocellular Carcinoma and Predict Tivantinib Sensitivity In Vitro. Clin. Cancer Res. [Internet]. 2017;Available from: http://dx.doi.org/10.1158/1078-0432.CCR-16-3118

162. Zhu AX, Baron AD, Malfertheiner P, Kudo M, Kawazoe S, Pezet D, et al. Ramucirumab as Second-Line Treatment in Patients With Advanced Hepatocellular Carcinoma: Analysis of REACH Trial Results by Child-Pugh Score. JAMA Oncol. 2017;3:235-243.

163. Kim RD, Sarker D, Meyer T, Yau T, Macarulla T, Park J-W, et al. First-in-Human Phase I Study of Fisogatinib (BLU-554) Validates Aberrant Fibroblast Growth Factor 19 Signaling as a Driver Event in Hepatocellular Carcinoma. Cancer Discov. [Internet]. 2019;Available from: http://dx.doi.org/10.1158/2159-8290.CD-19-0555 
164. Sia D, Jiao Y, Martinez-Quetglas I, Kuchuk O, Villacorta-Martin C, Castro de Moura $\mathrm{M}$, et al. Identification of an Immune-specific Class of Hepatocellular Carcinoma, Based on Molecular Features. Gastroenterology. 2017;153:812-826.

165. Finn RS, Kudo M, Cheng A-L, Wyrwicz L, Ngan R, Blanc J-F, et al. LBA30Analysis of serum biomarkers (BM) in patients (pts) from a phase 3 study of lenvatinib (LEN) vs sorafenib (SOR) as first-line treatment for unresectable hepatocellular carcinoma (uHCC). Ann. Oncol. [Internet]. 2017 [cited 2019 Jul 26];28. Available from: https://academic.oup.com/annonc/article-abstract/28/suppl_5/mdx440.022/4109934

166. Kaseb AO, Carmagnani Pestana R, Vence LM, Blando JM, Singh S, Ikoma N, et al. Randomized, open-label, perioperative phase II study evaluating nivolumab alone versus nivolumab plus ipilimumab in patients with resectable HCC. J. Clin. Orthod. 2019;37:185-185.

167. Kwak EL, Bang Y-J, Camidge DR, Shaw AT, Solomon B, Maki RG, et al. Anaplastic lymphoma kinase inhibition in non-small-cell lung cancer. N. Engl. J. Med. 2010;363:1693-1703.

168. Lynch TJ, Bell DW, Sordella R, Gurubhagavatula S, Okimoto RA, Brannigan BW, et al. Activating mutations in the epidermal growth factor receptor underlying responsiveness of non-small-cell lung cancer to gefitinib. N. Engl. J. Med. 2004;350:2129-2139.

169. Slamon DJ, Leyland-Jones B, Shak S, Fuchs H, Paton V, Bajamonde A, et al. Use of chemotherapy plus a monoclonal antibody against HER2 for metastatic breast cancer that overexpresses HER2. N. Engl. J. Med. 2001;344:783-792.

170. Finn RS, Martin M, Rugo HS, Jones S, Im S-A, Gelmon K, et al. Palbociclib and Letrozole in Advanced Breast Cancer. N. Engl. J. Med. 2016;375:1925-1936.

171. Demetri GD, von Mehren M, Blanke CD, Van den Abbeele AD, Eisenberg B, Roberts PJ, et al. Efficacy and safety of imatinib mesylate in advanced gastrointestinal stromal tumors. N. Engl. J. Med. 2002;347:472-480.

172. Druker BJ, Guilhot F, O'Brien SG, Gathmann I, Kantarjian H, Gattermann N, et al. Five-year follow-up of patients receiving imatinib for chronic myeloid leukemia. N. Engl. J. Med. 2006;355:2408-2417.

173. Pantel K. Blood-Based Analysis of Circulating Cell-Free DNA and Tumor Cells for Early Cancer Detection. PLoS Med. 2016;13:e1002205.

174. Mandrekar SJ, An M-W, Sargent DJ. A review of phase II trial designs for initial marker validation. Contemp. Clin. Trials. 2013;36:597-604.

175. Labgaa I, Villanueva A. Liquid biopsy in liver cancer. Discov. Med. 2015;19:263273.

176. Corcoran RB, Chabner BA. Application of Cell-free DNA Analysis to Cancer Treatment. N. Engl. J. Med. 2018;379:1754-1765.

177. Wan JCM, Massie C, Garcia-Corbacho J, Mouliere F, Brenton JD, Caldas C, et al. Liquid biopsies come of age: towards implementation of circulating tumour DNA. Nat. Rev. Cancer. 2017;17:223-238. 
178. Office of the Commissioner. FDA approves first blood test to detect gene mutation associated with non-small cell lung cancer [Internet]. 2016 [cited 2019 May 16];Available from: https://www.fda.gov/news-events/press-announcements/fdaapproves-first-blood-test-detect-gene-mutation-associated-non-small-cell-lung-cancer

179. Labgaa I, Villacorta-Martin C, D'Avola D, Craig AJ, von Felden J, Martins-Filho SN, et al. A pilot study of ultra-deep targeted sequencing of plasma DNA identifies driver mutations in hepatocellular carcinoma. Oncogene. 2018;37:3740-3752.

180. Qu C, Wang Y, Wang P, Chen K, Wang M, Zeng H, et al. Detection of early-stage hepatocellular carcinoma in asymptomatic HBsAg-seropositive individuals by liquid biopsy. Proc. Natl. Acad. Sci. U. S. A. [Internet]. 2019;Available from: http://dx.doi.org/10.1073/pnas.1819799116

181. Xu R-H, Wei W, Krawczyk M, Wang W, Luo H, Flagg K, et al. Circulating tumour DNA methylation markers for diagnosis and prognosis of hepatocellular carcinoma. Nat. Mater. 2017;16:1155-1161.

182. Bhan I, Mosesso K, Goyal L, Philipp J, Kalinich M, Franses JW, et al. Detection and Analysis of Circulating Epithelial Cells in Liquid Biopsies From Patients With Liver Disease. Gastroenterology [Internet]. 2018;Available from: http://dx.doi.org/10.1053/j.gastro.2018.09.020

183. Oh CR, Kong S-Y, Im H-S, Kim HJ, Kim MK, Yoon K-A, et al. Genome-wide copy number alteration and VEGFA amplification of circulating cell-free DNA as a biomarker in advanced hepatocellular carcinoma patients treated with Sorafenib. BMC Cancer. 2019;19:292.

184. Lim HY, Merle P, Weiss KH, Yau TC, Ross P, Mazzaferro V, et al. Phase II Studies with Refametinib or Refametinib plus Sorafenib in Patients with RAS-mutated Hepatocellular Carcinoma. Clin. Cancer Res. [Internet]. 2018;Available from: http://dx.doi.org/10.1158/1078-0432.CCR-17-3588

185. Chie W-C, Blazeby JM, Hsiao C-F, Chiu H-C, Poon RT, Mikoshiba N, et al. International cross-cultural field validation of an European Organization for Research and Treatment of Cancer questionnaire module for patients with primary liver cancer, the European Organization for Research and Treatment of Cancer quality-of-life questionnaire HCC18. Hepatology. 2012;55:1122-1129.

186. Blazeby JM, Currie E, Zee BCY, Chie W-C, Poon RT, Garden OJ, et al. Development of a questionnaire module to supplement the EORTC QLQ-C30 to assess quality of life in patients with hepatocellular carcinoma, the EORTC QLQHCC18. Eur. J. Cancer. 2004;40:2439-2444.

187. Huang G, Chen X, Lau WY, Shen F, Wang RY, Yuan SX, et al. Quality of life after surgical resection compared with radiofrequency ablation for small hepatocellular carcinomas. Br. J. Surg. 2014;101:1006-1015.

188. Salem R, Gilbertsen M, Butt Z, Memon K, Vouche M, Hickey R, et al. Increased quality of life among hepatocellular carcinoma patients treated with radioembolization, compared with chemoembolization. Clin. Gastroenterol. Hepatol. 2013;11:13581365.e1.

189. Kudo M, Trevisani F, Abou-Alfa GK, Rimassa L. Hepatocellular Carcinoma: 
Therapeutic Guidelines and Medical Treatment. Liver Cancer. 2016;6:16-26.

190. Omata M, Cheng A-L, Kokudo N, Kudo M, Lee JM, Jia J, et al. Asia-Pacific clinical practice guidelines on the management of hepatocellular carcinoma: a 2017 update. Hepatol. Int. 2017;11:317-370.

191. Kokudo N, Takemura N, Hasegawa K, Takayama T, Kubo S, Shimada M, et al. Clinical practice guidelines for hepatocellular carcinoma: The Japan Society of Hepatology 2017 (4th JSH-HCC guidelines) 2019 update. Hepatol. Res. [Internet]. 2019;Available from: http://dx.doi.org/10.1111/hepr.13411

192. Surveillance group, Diagnosis group, Staging group, Surgery group, Local ablation group, TACE/TARE/HAI group, et al. Management consensus guideline for hepatocellular carcinoma: 2016 updated by the Taiwan Liver Cancer Association and the Gastroenterological Society of Taiwan. J. Formos. Med. Assoc. 2018;117:381403.

193. Zhou J, Sun H-C, Wang Z, Cong W-M, Wang J-H, Zeng M-S, et al. Guidelines for Diagnosis and Treatment of Primary Liver Cancer in China (2017 Edition). Liver Cancer. 2018;7:235-260.

194. Korean Liver Cancer Association, National Cancer Center. 2018 Korean Liver Cancer Association-National Cancer Center Korea Practice Guidelines for the Management of Hepatocellular Carcinoma. Gut Liver. 2019;13:227-299.

195. Sharma $P$, Allison JP. The future of immune checkpoint therapy. Science. 2015;348:56-61.

196. Fukumura D, Kloepper J, Amoozgar Z, Duda DG, Jain RK. Enhancing cancer immunotherapy using antiangiogenics: opportunities and challenges. Nat. Rev. Clin. Oncol. 2018;15:325-340.

197. Kalbasi A, Ribas A. Tumour-intrinsic resistance to immune checkpoint blockade. Nat. Rev. Immunol. 2020;20:25-39.

198. Ruiz de Galarreta M, Bresnahan E, Molina-Sanchez P, Lindblad KE, Maier B, Sia $D$, et al. $\beta$-catenin activation promotes immune escape and resistance to anti-PD-1 therapy in hepatocellular carcinoma. Cancer Discov. [Internet]. 2019;Available from: http://dx.doi.org/10.1158/2159-8290.CD-19-0074

199. Pinyol R, Sia D, Llovet JM. Immune exclusion-Wnt/CTNNB1 class predicts resistance to immunotherapies in HCC. Clin. Cancer Res. [Internet]. 2019;Available from: http://dx.doi.org/10.1158/1078-0432.CCR-18-3778

200. Harding JJ, Nandakumar S, Armenia J, Khalil DN, Albano M, Ly M, et al. Prospective Genotyping of Hepatocellular Carcinoma: Clinical Implications of Next Generation Sequencing for Matching Patients to Targeted and Immune Therapies. Clin. Cancer Res. [Internet]. 2018;Available from: http://dx.doi.org/10.1158/10780432.CCR-18-2293 


\title{
Title: Trial Design and Endpoints in hepatocellular carcinoma: AASLD Consensus Conference.
}

Authors: Josep M. Llovet ${ }^{1,2,3}$ Augusto Villanueva1, Jorge A. Marrero4, Myron Schwartz¹, Tim Meyer ${ }^{5}$, Peter R. Galle ${ }^{6}$, Riccardo Lencioni ${ }^{7,8}$, Tim F. Greten ${ }^{9}$, Masatoshi Kudo ${ }^{10}$, Sumithra J. Mandrekar ${ }^{11}$, Andrew X. Zhu ${ }^{12,13}$, Richard S. Finn ${ }^{14}$, Lewis R. Roberts ${ }^{15}$, for AASLD Panel of experts on Trial Design in HCC.

\begin{abstract}
Affiliations: ${ }^{1}$ Mount Sinai Liver Cancer Program, Division of Liver Diseases, Tisch Cancer Institute, Icahn School of Medicine at Mount Sinai, New York, NY, USA; ${ }^{2}$ Translational Research in Hepatic Oncology, Liver Unit, IDIBAPS, Hospital Clinic, University of Barcelona, Catalonia, Spain; ${ }^{3}$ Institució Catalana d'Estudis Avançats (ICREA), Barcelona, Catalonia, Spain. ${ }^{4}$ UT Southwestern Medical Center, Dallas, Texas; ${ }^{5}$ Dept Oncology, University College London Cancer Institute; ${ }^{6}$. Dept. of Internal Medicine, Mainz University Medical Center, Mainz, Germany; '7Department of Radiology, University of Pisa School of Medicine; 8 Miami Cancer Institute, Miami, FL; ${ }^{9}$ Gastrointestinal Malignancy Section, Thoracic and Gastrointestinal Oncology Branch, Center for Cancer Research, National Cancer Institute, National Institutes of Health, Bethesda, Maryland; ${ }^{10 D e p a r t m e n t ~ o f ~}$ Gastroenterology and Hepatology, Kindai University Faculty of Medicine, Osaka-Sayama, Osaka, Japan. ${ }^{11}$ Department of Health Sciences Research, Mayo Clinic, Rochester, MN. ${ }_{12}$ Massachusetts General Hospital Cancer Center, Harvard Medical School, Boston, MA, USA; ${ }^{13}$ Jiahui International Cancer Center, Shanghai, China; ${ }^{14}$ Geffen School of Medicine at UCLA, California; ${ }^{15}$ Gastroenterology \& Hepatology Dept. Mayo Clinic, Rochester, MN.
\end{abstract}

Conflict of interest: Josep M Llovet is receiving research support from Bayer HealthCare Pharmaceuticals, Eisai Inc, Bristol-Myers Squibb, Ipsen and Boehringer-Ingelheim and consulting fees from Eisai Inc, Merck, Bayer HealthCare Pharmaceuticals, Bristol-Myers Squibb, Celsion Corporation, Eli Lilly, Ipsen, Glycotest, Roche, AstraZeneca, Sirtex, Nucleix and Can-Fite. Augusto Villanueva has received consulting fees from Guidepoint and Fujifilm; advisory board fees from Exact Sciences, Nucleix, Gilead and NGM Pharmaceuticals, and research support from Eisai Pharmaceuticals. Jorge Marrero reports consulting from Glycotest. Peter Galle is receiving research support from Bayer HealthCare Pharmaceuticals and consulting fees from Bayer HealthCare Pharmaceuticals, BristolMyers Squibb, Eisai Inc, Merck, Eli Lilly, Ipsen, Roche, AstraZeneca, Sirtex. Tim Meyer has received advisory board fees from Eisai, Roche, AstraZeneca, Ipsen, BTG and Tarveda. Masatoshi Kudo is receiving lecture fee from Eisai Inc, Bayer, MSD, research support from Eisai Inc, Gilead, Takeda, Otsuka, Taiho, Bristol-Myers Squibb, Ono Pharmaceutical and consulting fees from Bayer, Eisai, Ono, MSD, BMS, Eli Lilly, Roche, AstraZeneca, Chugai. Andrew Zhu reports research funding from Bayer, Bristol-Myers Squibb, Eli Lilly, Merck, and Novartis, and is a consultant/advisory board member for AstraZeneca, Bayer, Bristol-Myers Squibb, Eisai, Eli Lilly, Exelixis, Merck, Novartis, Roche, and Sanofi. Richard Finn reports consulting fees from AstraZeneca, Bayer, BristolMyersSquibb, CStone, Eisai, Eli Lilly, 
Merck, Novartis, Pfizer, Roche/Genentech. Lewis Roberts reports research funding from Bayer, BTG International, Exact Sciences, Gilead Sciences, Glycotest, Redhill Biopharma, TARGET PharmaSolutions, and Wako Diagnostics, and is a consultant/advisory board member for Bayer, Exact Sciences, Gilead Sciences, GRAIL, QED Therapeutics, and TAVEC.

All other co-authors do not have conflicts to disclose.

Acknowledgements: Josep M Llovet is supported by European Commission (EC)/Horizon 2020 Program (HEPCAR, Ref. 667273-2), EIT Health (CRISH2, Ref. 18053), Accelerator Award (CRUCK, AEEC, AIRC) (HUNTER, Ref. C9380/A26813), National Cancer Institute (P30-CA196521), U.S. Department of Defense (CA150272P3), Samuel Waxman Cancer Research Foundation, Spanish National Health Institute (SAF2016-76390) and the Generalitat de Catalunya/AGAUR (SGR-1358). Augusto Villanueva is supported by the Department of Defense (CA150272P3). Tim Greten is supported by the Intramural Research Program of the $\mathrm{NIH}$, National Cancer Institute, Center for Cancer Research. Sumithra J. Mandrekar is supported by a P30CA15083 grant. Andrew Zhu has received research support from V Foundation for Cancer Research, and DOD of Defense Team Science Translational Award. Richard Finn is supported by US Dept of Defense IDEA Award. Tim Meyer is supported by the NIHR UCLH Biomedical Research Centre. Lewis Roberts is supported by the National Cancer Institute (P50 CA210964).

Correspondence: Josep M. Llovet, MD, PhD, FAASLD. Mount Sinai Liver Cancer Program, Division of Liver Diseases, Tisch Cancer Institute, Icahn School of Medicine at Mount Sinai, Madison Ave 1425. 11F-70. Box 1123, New York, NY10029. USA. E-mail: josep.llovet@mssm.edu 


\begin{abstract}
Proper trial design is critical for the success of clinical investigations. Hepatocellular carcinoma (HCC) is a complex disease that has several unique properties. In 2008, after the approval of sorafenib, a panel of experts proposed guidelines for trial design and endpoints in HCC that have been instrumental during the last decade and provided a framework to allow an homogeneous analysis of reported investigations. Since then, several phase III studies have been reported and novel challenges have emerged. A panel of experts conveyed by AASLD organized a Special Topic Conference on trial design and endpoints to address those emerging challenges. This review summarizes the analysis and conclusions of those discussions and provides novel recommendations on the selection endpoints, stratification variables and targeted populations in the complex arena of HCC. We have covered the full spectrum of the disease, from surveillance/ chemoprevention, to neoadjuvant and adjuvant trials after curative therapies, and trials in intermediate and advanced stages of HCC. We explore the prospects for incorporating biomarkers and liquid biopsy into conventional clinical trials. In addition, we address the need for obtaining tissue and blood samples in all investigations and propose novel primary endpoints such as progression free survival with restrictive rules and patient reported outcomes. This up-dated set of recommendations is timely considering the advent of more potent combination therapies in all areas of HCC management, the increase in adverse events associated with those combinations, and the evidence that several lines of effective treatments will benefit a given patient. We herein articulate a framework to facilitate capturing the efficacy of novel therapeutic strategies with the goal of improving the outcomes of patients suffering from this disease.
\end{abstract}




\section{Introduction}

Hepatocellular carcinoma (HCC) is the fourth leading cause of cancer-related mortality worldwide $(1,2)$. This neoplasm has some unique characteristics. It occurs in most cases complicating underlying cirrhosis, has specific non-invasive criteria for diagnosis, follows a unique staging system and historically has been resistant to conventional chemotherapy. Several treatments have achieved adoption as standard of care according to clinical practice guidelines, including potentially curative therapies (i.e. resection, liver transplantation and local ablation) for early tumors, transarterial chemoembolization (TACE) for intermediate stage tumors, and systemic drugs for advanced tumors in front line (sorafenib and lenvatinib) and second line (regorafenib, cabozantinib and ramucirumab)(35). Life expectancy has improved progressively in all stages of the disease. Effective implementation of surveillance for patients at risk of developing HCC and access to current proven therapies has been a milestone. Overall, median survival times beyond 5 years are expected for early stages, about 20-30 months for intermediate stages, and between 10-16 months for advanced stage HCC [Fig. 1(3,4,6)]. Novel drugs and combinations continue to enter the research arena to address unmet medical needs. All these research activities require precise endpoints and tools for measuring clinical benefits.

Thus, clinical trial design has become a major focus of attention in HCC research. Since randomized controlled trials are the main source of evidence for drug approvals in oncology, it is of paramount relevance to understand the critical endpoints and tools for measuring them, as well as optimal strategies for identifying and enrolling target populations and for patient stratification. It has become evident that a deep understanding of factors determining HCC outcomes and trial design is required to achieve optimal results. There are trials with a non-inferiority design that have been positive, and lead to drug approval, while others demonstrate superior outcomes in surrogate end-points, such as progression free survival (PFS) or patient reported outcomes but not in the primary endpoint of overall survival. Some recent trials have also been reported to be statistically negative but "clinically positive". It is important to understand the reasons for the failure or success of a trial in order to move the field forward. In addition, while several positive phase III trials for advanced HCC have recently changed clinical practice (7-11), no major advances have occurred in the area of surveillance and early detection, adjuvant therapies after resection/ablation or management of intermediate stage HCC over the last 15 years. The lack of effective novel drugs/devices may be the cause of negative studies in these areas, but suboptimal trial design may also have jeopardized the likelihood of a positive result. With this challenge in mind, the Hepatobiliary Neoplasia Special Interest Group of the American Association for the Study of Liver Disease (AASLD) organized a Single Topic Conference in Atlanta in 2019 to address these issues. This position paper summarizes the major concepts discussed in the conference with the aim of updating the proposals previously reported by a similar AASLD panel in 2008(12).

\section{Overview on trial design and endpoints}

Clinical trials are essential to establish the clinical efficacy of new therapeutic interventions. They are instrumental in developing clinical practice guidelines and form the basis for evidence-based medicine(13). An adequate clinical trial design is crucial, as an effective drug can be discarded due to a poor trial design and vice-versa. The main considerations when designing a clinical trial are to: a) select a well-defined target patient population (i.e., inclusion and exclusion criteria), b) pre-specify clear endpoints (primary and secondary) and data analysis plan, c) specify randomization and allocation method; and d) secure efficacy of randomization (stratification at enrollment for prognostic variables). Based on these and other variables, the quality of clinical trials can be quantified using different scores such as 


\section{1}

the Jadad score(14), the Delphi List(15), the CONSORT statement(16,17), and the Cochrane Back Review Group criteria(18). Until the SHARP trial(11), which established the benefit of sorafenib in advanced stages HCC patients, the quality of the trials conducted in $\mathrm{HCC}$ was commonly modest. A systematic review found that only $50 \%$ of the clinical trials reported between 2002 and 2005 in HCC were deemed high quality as per the modified Jadad score(19). The 2008 position paper resulting from the AASLD conference provided a useful framework for academic centers, industry partners and regulators on the design of trials in $\mathrm{HCC}(12)$. Subsequently, the quality of clinical trials assessing systemic therapies has significantly improved. There has been less activity in terms of high-end clinical trials in other treatment areas. This position paper will extensively discuss the singularities of trial design in every clinical aspect of HCC management.

In clinical trials, the benefit of an intervention is quantified using endpoints, which are predefined events that once reached exclude the patient from further evaluation within the trial. There are 3 main types of endpoints: hard, surrogate and patient-reported, all extensively described elsewhere(20). Hard endpoints are well defined and easy to measure objectively. The archetypes of a hard endpoint are overall survival (OS) or cancer-related survival. Surrogate endpoints, such as progression-free survival or time to progression, partially rely on the quantification of tumor response, generally using imaging techniques and pre-specified criteria(21). Surrogate endpoints are more vulnerable than hard endpoints, but they have several advantages including their convenience in terms of event accumulation and trial feasibility. Patient-reported endpoints, sometimes referred as soft endpoints, are subjective measures such as quality of life (QoL), in most instances obtained from questionnaires. Overall recommendations of trial design and endpoints in HCC are detailed in Table 1, whereas expected outcomes for standard of care therapeutic interventions within these trials are summarized in Table 2.

\section{Endpoints}

OS is defined by the time between patient randomization and death from any cause. OS is usually recommended as the primary endpoint for randomized phase III clinical trials(12). OS is the endpoint most frequently used by regulatory agencies to approve drugs as it is objective and clinically relevant. In HCC, as most patients suffer from concomitant cirrhosis, death can result from competing risks, mainly liver toxicity and failure. This fact underscores the need for detailed assessments of safety with any intervention in this population. It is important to capture adverse events in early phase clinical studies as well as in larger randomized studies. Failures in phase 3 studies have been seen from new agents that are more toxic in an HCC population than in other tumor types(22). The competing risk of cirrhosis can introduce bias when evaluating the anti-tumoral activity of a therapeutic intervention, but it can be easily controlled by imposing stringent inclusion criteria in terms of liver function (i.e., Child-Pugh score A without hepatic decompensation). OS has some limitations such as the long follow-up time required to capture the number of events needed to verify significantly improved survival in the experimental arm(23). This can be a critical limitation when exploring interventions at early or intermediate stages. Also, OS can be confounded by sequential therapies received by patients after tumor progression, which for instance affected $30 \%$ of patients enrolled in the lenvatinib trial(7) and up to $50 \%$ of patients in the Checkmate 459 comparing nivolumab vs sorafenib(24). Hence, there is a need to develop surrogate endpoints, which are defined as outcomes not inherently meaningful from the clinical standpoint, but thought to accurately predict hard outcomes such as OS(25).

The main surrogate endpoints in oncology are progression-free survival (PFS), time-toprogression (TTP) and objective response rate (ORR). PFS is the time between patient 
randomization and death or radiological tumor progression, whichever occurs first. There are different tools assessing tumor response with imaging. The most established tool for measuring tumor response in oncology are the RECIST criteria(26), initially developed to evaluate response to cytotoxic drugs. These criteria were adapted to account for HCC singularities in the modified RECIST (mRECIST) version, which incorporates viable tumour detected with arterial enhancement as a key component to evaluate response $(21,27)$. Using mRECIST criteria increases the percentage of subjects who achieve objective response compared to standard RECIST, as shown in different studies of systemic therapies (28-32). A recent meta-analysis evaluated the power of PFS to predict OS in phase 3 trials testing systemic therapies in advanced $\mathrm{HCC}(20)$. The study found a moderate correlation between PFS and OS in 21 RCTs. The authors proposed a conservative surrogate threshold of $\leq 0.6$ for hazard ratio of PFS to predict clinically relevant improvements in OS(33). TTP is defined as the time elapsed between patient randomization and radiological tumor progression. Scheduling repeated radiological assessment of response every 6-8 weeks is mandatory for patients included in trials. Data from SHARP and subsequent studies challenges the implied correlation between TTP and OS. The type of progression may also have clinical implications (34). Survival is worse if patients develop a new extrahepatic lesion and/or vascular invasion as opposed to tumor progression resulting from growth of an existing lesion or a new intrahepatic lesion. Lastly, ORR is the percentage of patients with an objective tumor response, and its correlation with OS is worse than for PFS or TTP(20). This is partially inherent to the use of odds ratios instead of hazard ratios for ORR and also to the fact that only a small proportion of patients achieve an objective response $(<25 \%$, for approved drugs in advanced HCC), which is the event that correlates with OS(31). Nonetheless, ORR has been reported as an independent predictor of survival in early HCC treated with radiofrequency ablation, intermediate treated with TACE and advanced HCC treated with $\mathrm{TKI}(27)$. The impact of the duration of response, which has been reported to be around 12 months for checkpoint inhibitors vs. less than 6 months for TKIs, has not yet been properly incorporated into response assessment. The same is true to small reductions in tumor size not reaching standard thresholds for objective response. In some cases, duration of disease control may be more clinically relevant than the extent of reduction in tumor size. Also, in the case of immune checkpoint inhibitors, tumor response can have a longer lagtime compared to other molecular therapies and can even mimic progression shortly after treatment initiation (i.e., pseudo-progression(35)). This has led to the development of immune-related response criteria(36), which require confirmation of progression at least 4 weeks after progressive disease is first documented.

Surrogate endpoints are frequently used by the Food and Drug Administration (FDA) to approve drugs under the accelerated program, which was initially developed to facilitate early access to new antivirals during the worst years of the HIV epidemic(37). In HCC, the FDA has used ORR and duration of response to grant accelerated approval of the immune checkpoint inhibitors (CPI) nivolumab(38), pembrolizumab(39) and recently the combination of ipilimumab and nivolumab(40). Accelerated approval is not universal and includes some subjectivity from regulators in regards to the strength of the evidence to support approval without a randomized phase 3 study $(24,41)$. In addition, while the use of ORR and other surrogate endpoints may be used to support regulatory approval, they do not necessarily support inclusion in guidelines which often adhere to a higher level of evidence. Despite their common use, surrogate endpoints are vulnerable to interpretation bias. Besides the strength of the endpoint, it is key to determine when the benefit provided by a new therapy is really clinically meaningful. This can be controversial, depending on factors such as the perceptions of patients, providers, health insurers and regulators. In HCC, there is no set threshold that defines a clinically meaningful benefit, but some authors have suggested a hazard ratio cutoff of OS $\leq 0.8$ as a starting point for clinical trial design(42). In fact, all 
positive trials in HCC have led to significant differences in survival with HR below this threshold.

\section{Surveillance for Hepatocellular Carcinoma: design and endpoints}

Surveillance for HCC is one of the milestones advancing the management of HCC, despite that there is not unquestionable data directly supporting a decrease in cancer-related death in persons on surveillance(43). Ultrasound (US), with or without alpha-fetoprotein (AFP), performed every six months is the current standard and is recommended for surveillance of patients with cirrhosis of any cause or chronic hepatitis B without cirrhosis above a regional and gender appropriate age cut-off determined by expert liver societies $(3,4)$. Overall, the implementation of those programs to all targeted populations is modest, and current data report detection of HCC in the setting of surveillance in between $30-50 \%$ of cases(44). In meta-analysis, the pooled sensitivity and specificity of US alone has been shown to be $53 \%$ (95\% Cl: 35-70) and 91\% (95\% Cl:86-94), respectively, while the combination of US and AFP has a sensitivity of $63 \%(95 \% \mathrm{Cl}: 48-75)$ and a specificity of $85 \%(95 \% \mathrm{Cl}: 77-89)(45)$. Due to the relatively low sensitivity and specificity of this approach for detecting early stage HCC, particularly in North America, where high rates of central obesity decrease the performance of ultrasound, a recent study showed that this strategy leads to $27 \%$ of patients with cirrhosis experiencing harms such as follow up testing (CT, MRI, liver biopsy) performed for false-positive or indeterminate results(46). Further, due to low implementation of comprehensive strategies for HCC surveillance, more than $60 \%$ of HCCs in North America, Europe, Africa and large parts of Asia, excepting Taiwan and Japan, are diagnosed with intermediate or advanced stage $\mathrm{HCC}(47)$. There is, therefore, an urgent need for better performing, low cost surveillance strategies in $\mathrm{HCC}$, and accounting for both the benefits and harms of surveillance strategies is important.

Within this overriding context, there is excitement time because advances in genetic, epigenetic, proteomic, glycoproteomic and metabolomic analyses in have enabled large scale multi-omic analyses of HCC tissues, circulating tumor DNA, plasma and serum, resulting in the accelerated identification of novel biomarkers(48-51). Models using standard biostatistical and machine learning and artificial intelligence ( $\mathrm{Al}$ ) approaches are using biomarkers combined with clinical parameters to identify persons at highest risk for HCC. Models and biomarkers under active exploration include the GALAD (Gender, Age, AFP-L3, AFP, and Des-carboxy-prothrombin) score(52), novel glycoproteins (fucosylated kininogen)(53), liquid biopsy analyses of circulating tumor DNA for differentially methylated regions(54)(55), and imaging with abbreviated $\mathrm{MRI}(56)$. Creating the framework for validation of future surveillance is critically important.

To guide the development and evaluation of new surveillance strategies for clinical utilization, a 5-phase program has been developed by the $\mathrm{NCl}$ - Early Detection Research Network (EDRN) for biomarker that uses human samples (blood or human tissue) as well as imaging tests (57). Table 3 shows recommended phases of surveillance test validation, including trial design for studies for HCC surveillance. Phase 1 are biomarker discovery or exploratory studies. Phase 2 studies estimate the ability of a test to distinguish early stage HCC from those with cirrhosis without HCC. It is important to test for confounders such as age, etiology of liver disease, and liver function; and to have adequate sample size and power. Phase 3 studies enroll at risk individuals and follow them for clinical diagnosis of HCC using prospective-specimen collection, retrospective-blinded evaluation (PRoBE) design (58). The aim is to evaluate, as a function of time before clinical diagnosis, the capacity of the test to detect preclinical HCC; and also, to define the criteria for a positive surveillance test in preparation for phase 4 and 5 studies. Thus, Phases 1-3 rely on retrospective analysis of stored data and specimens. Phase 4 studies require the new test 
be applied to patients with cirrhosis in the clinical setting to assess test performance in HCC detection and false positive and negative rates. Depending on the test under study it may be possible to skip Phase 4 if the test is already used for patient care, for example, evaluating an MRI for surveillance of HCC. Phase 5 studies are randomized trials comparing the new surveillance tests against the standard of care, in the case of HCC the standard should be US with or without AFP, with the aim of determining whether the test can reduce mortality at the population level.

When performing surveillance studies in patients with cirrhosis it is important to enrich the at risk population in order to achieve a sufficient number of incident HCCs in a reasonable time period. Enriching cohorts with patients of older age, viral hepatitis, male sex, Hispanic ethnicity, history of diabetes, and family history of HCC should be considered $(59,60)$. Alternatively, known independent factors associated with HCC development are abnormal bilirubin and platelet count $<100,000 / \mathrm{mm}^{3}$. There is also a need to study currently important populations such as those with non-alcoholic fatty liver disease-related cirrhosis, those with hepatitis C-related cirrhosis who have achieved a sustained virological response after antiviral treatment, and those with suppressed hepatitis B infection on antiviral treatment. These three specific populations will be the most important etiological risk factors in the next decade and their HCC incidence rates (around 1\%/year) appear lower than in previous at risk populations(61). Methods for risk stratification within these populations will therefore become increasingly important for improving the effectiveness of surveillance strategies and programs. Models such as the REAL-B and PAGE-B scores, incorporating male sex, age, alcohol use, baseline cirrhosis, diabetes, platelet count and AFP, allow improved risk stratification of patients on oral antiviral therapy for chronic hepatitis B and could potentially be incorporated into surveillance programs(62).

An important potential confounder in studies that compare the performance of novel biomarkers to current surveillance strategies is the incorporation of imaging by ultrasound or other radiologic modalities into the standard of care. This may confound the results if ultrasound is also used as part of the control arm for the study, as ultrasonography is itself typically part of the gold standard process for determining whether a patient has HCC. Thus, patients with HCCs that are not visible by ultrasound may be falsely determined to be negative for cancer and a positive biomarker test erroneously labeled as a false positive. It is therefore important to use a different high-accuracy imaging modality such as multiphasic $\mathrm{MRI}$ as a gold standard in studies for which ultrasonography is part of the surveillance strategy. However, use of MRI may add substantial cost to the study and may also result in visualization of a number of small indeterminate false positive lesions that are seen on MRI and require follow up investigation, a component of the harms associated with surveillance. While studies of the performance of ultrasound with or without AFP in the clinical care setting have shown suboptimal performance in detection of HCC in at risk individuals, it is not clear what the performance characteristics are for phase 2, 3 or 4 biomarker studies that would meet the threshold for FDA approval as a surveillance test. In general, the FDA guidelines for supporting biomarker qualification recommend that analyses intended to support biomarker qualification should be specified in an analysis plan with a prospectiveretrospective design before analyzing the data. The FDA provides no set quantitative criteria for determining the relationship between the biomarker and clinical outcome, such as diagnosis of HCC, within a particular context of use. Overall, the goals for in vitro diagnostic biomarker studies are that they should produce valid scientific evidence demonstrating reasonable assurance of the safety and effectiveness of the product, and protect the rights and welfare of study subjects $(63,64)$.

Key unmet needs in the field of chemoprevention include an improved understanding of the potential for $\mathrm{HCC}$ risk reduction by chemoprevention using commonly used medications 
such as aspirin and other antiplatelet agents, statins, metformin and similar agents(65-68). In order to build a robust evidence base through chemoprevention trials, a number of key hurdles need to be crossed, including better definition of target populations, trial enrichment or stratification prior to randomization using clinical, genetic, or other molecular risk stratifying strategies, and careful delineation of appropriate and clinically meaningful endpoints for both biomarker-based and chemoprevention trials. Enrichment of populations included in chemopreventive trials should aim to a reasonable time-to event (occurrence of $\mathrm{HCC}$ ) endpoint, certainly within the threshold of 5 years. Stratification factors for at risk populations have been outlined below and are mandatory to prevent imbalances. Finally, one of the bottlenecks of these trials is that the accepted adverse events for maximum tolerated doses (grade 3 toxicities are unacceptable) are completely different compared to those accepted for primary treatments of advanced tumors, where grade 3-4 adverse events at the level of $30-50 \%$ are common for currently accepted drug treatments.

\section{Early HCC stages: design of trials for resection, transplantation and local ablation}

Hepatic resection is the treatment of choice for patients with preserved liver function (Child's class $\mathrm{A}$, bilirubin $<1.0 \mathrm{mg} / \mathrm{dl}$, no evidence of portal hypertension) who have a solitary HCC $>2 \mathrm{~cm}$ without macrovascular invasion(3,4,69) (Fig 1, Table 4). Outcomes of ideal candidates treated following these criteria are significantly better compared with outcomes not following the guidelines (70). Recent guidelines accepted expanding criteria to include patients with HCC within Milan criteria (3). While 5-year survival rates are in the range of $70 \%$ after resection, recurrence of $\mathrm{HCC}$ is also around $70 \%$ at 5 years)(71). Early (within 2 years) recurrence is most commonly due to the appearance of preexisting undetected metastatic disease, with the most common site in the remaining liver; late recurrence is predominantly the result of de novo development of $\mathrm{HCC}$ in the remaining liver. There is, thus, a critical unmet need for therapy that can reduce the incidence of HCC recurrence after resection.. A study demonstrating benefit of retinoid administration(72) was not confirmed in a subsequent multicenter trial(73), and small studies suggesting benefit from adoptive immunotherapy(74) and I-131 lipiodol embolization of the liver remnant(75) the results of which have not been duplicated. To this point, all phase III high-quality adjuvant trials conducted so far in this area have been negative, A large randomized, controlled trial of sorafenib after resection or thermal ablation demonstrated no benefit(76). Current attention is largely focused on immunotherapy. Treatment of advanced HCC with anti-PD1 or PD-L1 antibodies has consistently yielded responses in the range of $15-20 \%((38,39,41)$ that are often quite durable. In non-small cell lung cancer similar response rates are seen in advanced disease, and a neoadjuvant trial for resectable tumors resulted in a roughly doubled response rate(77).

Phase 3 trials are currently underway with single-agent immunotherapy or combination therapies. In advanced disease combination therapy, an anti-PD-1/PD-L1 plus either a tyrosine kinase inhibitor (e.g. sorafenib, lenvatinib), an anti-VEGF antibody (e.g. bevacizumab), or a second checkpoint inhibitor (e.g., anti-CTLA-4 antibody) appears to significantly raise response rates, and if established in the advanced setting combination therapy will no doubt be studied in adjuvant/neoadjuvant trials. The ultimate hope is that effective adjuvant/neoadjuvant therapy will be able to substantially improve recurrence-free survival. It is the consensus of the panel that entry criteria for adjuvant/neoadjuvant studies in HCC resection should conform to the criteria for resectability currently espoused in AASLD guidelines $(4,69)$, and prevent a broadening of the tumor eligibility for resection (e.g., multiple tumors, presence of vascular invasion) observed in some currently-running adjuvant trials. While all patients undergoing resection for HCC have significant risk of recurrence, studies should stratify for known risk factors including tumor size $(>3 \mathrm{~cm})$, microvascular invasion, differentiation degree and serum AFP $>400 \mathrm{ng} / \mathrm{mL}$. Neoadjuvant 
studies provide a unique opportunity to better understand what factors are associated with response to immunotherapy or lack thereof. Pretreatment biopsy should be mandatory, and thorough characterization of the tumor immune microenvironment should be built into these trials.

Liver transplantation is the treatment of choice for HCC within Milan criteria in patients who are not candidates for resection (78) (Fig.1, Table 4). These criteria lead to median OS of 10 years and recurrence rate of $<20 \%$. In the US it has been accepted that patients with more extensive disease ( one nodule between $5-8 \mathrm{~cm}$, $2-3$ nodules $\leq 5 \mathrm{~cm}$ or $4-5$ nodules < $3 \mathrm{~cm}$ with sum of diameters $<8 \mathrm{~cm}$ ) down-staged to Milan criteria are acceptable for transplantation (79). Downstaging is not accepted by European guidelines, although performed in some countries such as Italy. A significant number of patients who enter the waiting list or a down-staging protocol drop out and do not ultimately undergo transplantation. Locoregional therapy using thermal ablation or transarterial chemoembolization have been the modalities traditionally applied to maintain HCC within Milan criteria while awaiting transplant or to down-stage patients to eligibility. With the advent of effective systemic therapies, their role in the pretransplant setting vis-à-vis locoregional treatment warrants exploration in clinical trials. Locoregional treatment should be the control arm, compared to systemic therapy either alone or in combination with locoregional, with the primary endpoint of drop-out / transplantability. Stratification should be according to whether patients were initially within or beyond Milan criteria, or downstaged to Milan, and base-line AFP levels $>400 \mathrm{ng} / \mathrm{ml}$.

Treatment of HCC recurrence following transplantation is largely unstudied. The rate of recurrence in properly selected patients is low (10-20\%) and these patients have been routinely excluded from studies of systemic therapies. Tyrosine kinase inhibitors have been shown to be safe and are commonly used in an uncontrolled manner(80). There is considerable reluctance to use immunotherapy with anti-PD-1/L1 antibodies due to reports of treatment-related organ rejection, though there are reports of successful treatment(81). As HCC now accounts for nearly $25 \%$ of liver transplants in the US, it is time for trials to be implemented studying treatment of post-transplant HCC recurrence.

Local ablation is the mainstay treatment for nonsurgical candidates with early stage HCC $(3,4)$ (Fig 1, Table 4). Tumor size (up to 4-5 cm), number (up to 3 tumors) and location (accessiblility with ultrasound, CT or MRI guidance) limit the applicability of percutaneous ablation. Several randomized studies have demonstrated a significant benefit of radiofrequency ablation (RFA) over percutaneous ethanol injection in terms of complete response rate, and time to recurrence $(82,83)$. Consequently, RFA is the standard ablative therapy at early stages (Table 1). Median OS with RFA is of 60 months, with a recurrence rate ranging from $50-70 \%(3,4,82,83)$. AASLD and EASL guidelines have adopted radiofrequency ablation as front line therapy for single tumors $<2 \mathrm{~cm}$, but in tumors beyond this threshold resection remains as first treatment option(3,4). Randomized phase III trials are scarce in this arena, and are mostly currently focused on adjuvant therapies to prevent recurrence than in challenging the abaltive treatment. Microwave ablation has largely supplanted RFA in the United States(84), whereas ethanol injection is restricted to HCC < $2 \mathrm{~cm}$ in difficult locations. Cryoablation and irreversible electroporation are still under investigation $(3,4,85)$. Clinical benefit associated with the use of thermally-sensitive carriers loaded with liposomal doxorubicin in conjunction with radiofrequency ablation is currently tested in phase III.

Overall, the main criteria for trial design in the neo-adjuvant/adjuvant after resection/local ablation or liver transplantation setting are as follows (Table 1): 
1. Target populations for neoadjuvant and adjuvant trials: For resection, trials should include patients meeting current AASLD guidelines, and should not include patients with more advanced $\mathrm{HCC}$, e.g. macrovascular invasion. For transplantation, trials should include patients meeting criteria for listing (i.e., Milan criteria), or meeting established criteria for entry into downstaging protocols. For local ablation the target population should follow AASLD guidelines.

2. Endpoints: The appropriate end-point for adjuvant trials in the setting of either resection or transplant is recurrence-free or time to recurrence. For neo-adjuvant trials, pathological response or 1-yr recurrence can also be considered. For treatments challenging locoregional therapies, OS remains the primary endpoint, but PFS is also recommended as coprimary end point. Secondary endpoints should at least include objective response rates.

3. Stratification prior to randomization: Appropriate stratification parameters for neoadjuvant/adjuvant studies in the setting of early-stage HCC should include geographical region, tumor size and number, AFP $>400 \mathrm{ng} / \mathrm{mL}$, type of curative treatment, and pathological features of high risk (size $>3 \mathrm{~cm}$, microvascular invasion, differentiation degree and tumor satellites).

4. Control arms: For neoadjuvant/adjuvant studies in the setting of resection, a placebo control arm is appropriate. Adjuvant studies in transplantation should also include placebo controls. Defining the control arm for neoadjuvant studies in transplantation remains problematic as there is no evidence-based standard, but there is a general acceptance of the need to include loco-regional therapies to limit tumor progression in patients awaiting transplant that precludes including placebo or untreated patients. Control arms for devices or drugs challenging local ablation should be radiofrequency. Of note, since RFA has been considered effective in nodules up to $4 \mathrm{~cm}$, trials exploring treatments for single nodules beyond this size should consider chemoembolization as the best standard control.

5. Unmet needs: HCC recurrence rates after resection or local ablation are unacceptably high. Key needs include biomarkers to improve case selection, and effective neoadjuvant/adjuvant therapies. With regard to transplantation for HCC key needs include definition of optimal neoadjuvant (waiting list) strategies, and identification of useful biomarkers to refine candidate selection beyond algorithms based on tumor size and number.

\section{Trial design and endpoints in intermediate stage HCC}

TACE was established as the standard of care for intermediate stage HCC in 2002 following the publication of two small, randomized controlled trials for which OS was the primary endpoint (Table 4). The first trial, conducted in Barcelona, demonstrated a hazard ratio of 0.47 [95\% Cl 0.25-0.91], p=0.025) in favor of TACE, and a 2 year survival of $63 \%$ compared with $23 \%$ for supportive care(86). In the second, TACE was associated with an improvement in 2 year survival from $11 \%$ with supportive care to $31 \%$ with TACE, and a reduction in relative risk of death; $0.49(95 \% \mathrm{Cl}, 0.29-0.81 ; \mathrm{P}=0.006)(87)$. Response using WHO criteria, was evaluated as a secondary endpoint and was shown to be associated with a better survival(86). On the basis of these trials and a subsequent meta-analysis(88), the BCLC algorithm recommends TACE for those with intermediate stage disease HCC defined by liver confined, multinodular disease, in those patients with a performance status of 0 , Child Pugh A or B cirrhosis and in the absence of portal vein invasion(3,5) (Fig 1). Chemoembolization was subsequently adopted by AASLD and EASL guidelines of management of HCC, and no other therapy has so far replaced this standard of care. However, since 2003(86,88) there have been further innovations, guidelines and therapeutic advances which need to be considered in the design of current and future trials. Finally, radioembolization with Y90 for intermediate HCC has produced positive efficacy 
signals coming from phase 2 investigations(89), but they have not been adopted by guidelines awaiting phase 3 positive data for this specific population.

Eligibility criteria and stratification factors.

It is increasingly recognized that the BCLC B stage is heterogeneous and this likely accounts for the wide spectrum of reported survival outcomes, which range from 12-48 months. Consequently, there have been several proposals to subdivide the BCLC group but to date, none have been widely adopted(90,91). Additionally, patients who have a performance status of 1 but otherwise conform to the BCLC criteria, are routinely treated with TACE, and many clinicians regard Child-Pugh $B$ disease as a relative contraindication. Applicability of TACE in BCLC-B is $50 \%$, with the excluded patients having relative contraindications for the procedure due to advanced liver dysfunction or technical issues(92). Recent large RCTs have included patients with PS $0-1$, Child-Pugh $A$, and absence of portal vein thrombosis (Table 1,2)(93-96). Stratification factors have been less consistent with the exception of AFP for which a threshold of $400 \mathrm{ng} / \mathrm{ml}$ has been commonly applied. Composite and fully objective prognostic systems may provide a more feasible and consistent method by which to stratify patients. The ALBI score allocates a grade based on bilirubin and albumin and provides a more objective measure of liver function as compared with Child-Pugh class(97). A direct comparison between ALBI and Child Pugh has shown that the ALBI grade 1 is $92 \%$ Child-Pugh A5, ALBI 2 spans a wide range from A5 to B9 and ALBI 3 is B7 and above(98). However, tumor characteristics such as size and AFP are also prognostic and this has been addressed by the HAP score which provides a four class prognostic system using bilirubin, albumin, tumour size and AFP as categorical variables(99). The HAP score has been validated in the TACE-treated population, most recently within a cohort of 3000 patients(100). Applying the HAP score resulted in four distinct groups with survival ranging from 33 months for HAP A to 12 months for HAP D. HAP appears to be a simple and robust stratification factor that might be incorporated into TACE trials

\section{$\underline{\text { TACE procedure }}$}

The TACE technique provides another source of heterogeneity and potential bias(101). There remains no consensus regarding the optimal embolic particle, the role of lipiodol or the type of chemotherapy used. Indeed, there are no trials demonstrating the superiority of TACE over bland particle embolization (TAE) and a meta-analysis of five trials including 582 patients showed no difference in survival(102). It is unlikely that further technical innovation to the TACE procedure will result in significantly improved outcomes and the future generation of TACE trials will continue to evaluate the combination of TACE and systemic therapy or to compare TACE with systemic therapy. In both cases, TACE will be the control arm and it is important that this is standardized. To achieve this, some of the recent randomized trials have mandated use of drug-eluting beads (DEB TACE) $(93,94)$. Trials comparing DEB TACE with conventional TACE (CTACE) have failed to show a survival benefit but systemic toxicity from chemotherapy is reduced with DEB TACE(103) (104). If technique is not standardized, stratification according to center is an alternative way to reduce bias. Another area of contention is the schedule of TACE administration. In clinical practice, TACE is usually performed on demand according to radiological response rather than according to a fixed interval, and it is reasonable to recapitulate this in clinical trials. However, an effective systemic therapy may reduce the requirement for TACE. In the TACE-2 trial, there were $18 \%$ fewer TACE procedures performed in 12 months in the sorafenib arm compared with the placebo arm(93), and in the Oriental trial, the median number of procedures was 3.2 versus 3.7 in the orantinib and placebo arm respectively(96). Recording the number of procedures over the first 12 months or the mean number of procedures should be considered as a secondary endpoint for randomized trials of TACE 
versus TACE plus systemic therapy. In this sense, the reduction in frequency and number of TACE procedures may have implications for health economics and preservation of liver function.

\section{Response assessment}

Radiological response is an important indicator of therapeutic activity and can be a surrogate marker of long-term outcomes. Response assessment has been addressed in the next section, but few concepts regarding loco-regional therapies are summarized here. In TACE-related population, mRECIST demonstrated a higher response rate compared with RECIST 1.1(105). Moreover, there was a significant association between survival and overall response according to $\mathrm{MRECIST}$ but not with RECIST 1.1. The association between mRECIST response and survival has subsequently been confirmed in multiple other studies and a recent meta-analysis of seven studies including 1357 patients reported a hazard ratio for survival of $0.39(95 \% \mathrm{Cl} ; 0.26,0.61)$ for those with mRECIST response(106). Unfortunately, not all the recently reported phase 3 studies reported response and only TACE-2 ascertained response by both RECIST 1.1 and mRECIST. Best response by RECIST 1.1 was higher than first response but still less than response by mRECIST. Guidelines recommend capturing response as per mRECIST in clinical practice and both RECIST 1.1 and mRECIST as secondary endpoints trials targeting intermediate stage tumors(3).

\section{Primary endpoints}

In recent trials, OS for intermediate stage patients receiving TACE was of 21-33 months(9396) (Table 2). Over the past 10 years, there have been major advances in systemic therapy and many patients now transition from TACE to first and increasingly second line systemic therapy. In TACE-2, patients were unblinded on progression and $36 \%$ of those on placebo subsequently received sorafenib(93). Similarly, in the BRISK TA trial, $21 \%$ of placebo treated patients had post-progression systemic therapy (95) trial, and in the ORIENTAL trial, $66 \%$ of patients in the placebo arm received post-study therapy (96). Use of postprogression therapy may confound OS as an endpoint and increases the duration of followup required to meet the survival endpoint. To address this, a variety of surrogate endpoints have been proposed including progression free survival (PFS), time to progression (TTP), time to disease progression (TTDP), time to extrahepatic spread and vascular invasion (TTES/VI) and time to unTACEable progression (TTUP). Recent trials reporting these potential surrogates in addition to survival has allowed evaluation of their performance. The BRISK TA trial reported a promising hazard ratio of 0.61 for TTP but the trial missed its primary endpoint for survival (HR 0.9)(95). Overall, the correlation coefficient of TTP and OS is 0.77. A major limitation of TTP is that it fails to capture death, which is an important indication of toxicity as well as lack of efficacy. By contrast, PFS, which is the most commonly applied surrogate endpoint used in oncology, captures disease progression and death, and has been reported to correlate with OS in the TACE 2 trial. Novel composite endpoints have also been explored. Time to appearance of extrahepatic spread or vascular invasion (TTES/VI or MVI/EHS) showed a promising HRs of 0.64 and 0.62 in the BRISKT TA and SPACE trial that did not correlate with OS benefit $(94,95)$. Particularly, TTUP (time to untreatable progression), a composite end point defined as failure of response after to treatments, or emerging contraindications for TACE was tested in the SPACE trial, but failed to identified benefits for the combo of TACE plus sorafenib vs TACE (HR: 1.586). Recently, other novel endpoints were incorporated into the TACTICS trial comparing TACE plus sorafenib vs TACE alone(107) (108). In this study, PFS and OS were co-primary end points but progression was defined as unTACEable progression and Response Evaluation Criteria in Cancer of the Liver (RECICL)(109) was used to define progression rather than RECSIT 1.1 or mRECIST. Applying these criteria, PFS was superior in the combination arm 
$(\mathrm{HR}=0.59 ; 95 \% \mathrm{Cl}, 0.41$ to $0.87 ; \mathrm{p}=0.006)$ but further follow-up is required to establish wether this translates into a survival benefit. In the meantime, for RCT testing devices alone or in combination with systemic therapies it is recommended that PFS should be the coprimary endpoint along with OS, while ORR should be included as a secondary endpoint (Table 1 \& 2). Additional composite endpoints can be included as exploratory endpoints until they are properly validated.

A challenging question for the future is how TACE compares to systemic therapy. TACE was developed at a time when systemic therapy was virtually non-existent. With the advent of first, second and even third line systemic therapies and achieved OS beyond 2 years in selected patients receiving two lines of therapy(23), systemic therapy can be discussed not only following TACE but as an alternative to TACE. This is particularly relevant as transarterial therapies impair liver function and may render many patients no longer eligible for systemic therapy. For patients with limited tumor burden and nodules accessible superselectively by TACE, locoregional TACE may still be the best approach. In contrast, patients exceeding the up-to-seven criteria may be better suited for clinical trials exploring upfront systemic therapy(110). To answer this question a head-to-head comparison of TACE vs. systemic therapy (or vs. TACE plus systemic therapy) in defined patient subgroups will be needed, making the endpoint discussion even more complex.

\section{Radiologic assessment of response}

The RECIST criteria are the standard imaging approach for assessing tumor response in oncology. The original RECIST panel acknowledged that amendments could be needed for tumors with unique complexities and for evaluating non-cytotoxic drugs(111). Both issues are highly relevant for HCC: (a) the association of HCC with an underlying chronic liver disease complicates image assessment, since pathologic and hemodynamic changes in cirrhosis and extrahepatic manifestations of chronic liver disease may mimic tumor progression; (b) nonsurgical treatments for $\mathrm{HCC}$, including loco-regional and systemic therapies, achieve improvements in survival without inducing sizeable tumor shrinkage, frustrating attempts to capture tumor response using standard RECIST metrics(12).

In 2010, modified RECIST (mRECIST) criteria for HCC were proposed(21) addressing confounding factors related to cirrhosis using specific amendments for the assessment of lymph nodes, ascites, portal vein thrombosis, and newly detected hepatic nodules (Table 5). These recommendations were made primarily to prevent "overcalls" of progressive disease. In addition, the absence of substantial tumor shrinkage was addressed by introducing the concept of "viable tumor" in the measurement of intrahepatic HCC lesions, enabling the classification of treatment induced intratumoral necrosis in the absence of significant changes in overall tumor diameter as objective responses (21).

During the past decade, mRECIST for HCC has been used extensively in HCC clinical research and its performance has been reviewed elsewhere(27). The proposed mRECIST refinements for assessment of lymph nodes, ascites, portal vein thrombosis, and newly detected hepatic nodules, were progressively incorporated into radiology charters of HCC clinical trials, even when the criteria were named RECIST or RECIST 1.1(112). This process homogenized radiologic interpretation of these findings, improving consistency and reliability in assessment of tumor progression. Consequently, recent studies reported similar results for standard RECIST 1.1 and mRECIST in assessment of progression-driven endpoints, such as PFS and $\operatorname{TTP}(7,8)$. Currently, the main difference between standard RECIST and mRECIST is the approach to measurement of intrahepatic lesions, which primarily affects the ability to capture an objective response (OR). Use of the mRECIST viable tumor concept results in identification of 2-3 times more responders than standard 
RECIST, not only in patients receiving loco-regional treatments but also those receiving systemic therapies(7,32).

With the advent of immune checkpoint inhibitors, changes to the RECIST model have been proposed(35,36,113-115). Response to immunotherapy can manifest after imaging features that meet current RECIST criteria for progression. Pseudo-progression has been defined as increase in tumor size of existing lesions or the appearance of new lesions, followed by a response(35). Differentiating pseudo-progression from true progression is a challenging but important: while early discontinuation of an effective drug is not desirable, continued long-term treatment with a non-effective drug past true progression might delay the initiation of potentially effective therapies. Pseudo-progression has been described as a marginal event in phase III investigations with anti PDL1/PD1 check point inhibitors in HCC. The incidence of this phenomenon with anti- CTLA-4 and other inhibitors is unknown.

Limited information is available on use of immune-related criteria in HCC. In a phase II study of 104 patients who received pembrolizumab in second line after sorafenib, the use of immune-related RECIST (irRECIST) did not affect response rate or time to response as compared to mRECIST; however median PFS was 7.0 months $(95 \% \mathrm{Cl}, 4.9-8.0)$ when assessed by irRECIST vs 3.2 months (95\% CI, 2.2-4.1) when assessed by mRECIST(116). In phase Ilb study(117) investigating a vaccinia virus-based oncolytic immunotherapy pexastimogene devacirepvec- in advanced HCC changes to $\mathrm{mRECIST}$ were implemented because the treatment induces a flare with swelling and edema(118). These changes included the confirmation of progression at 4 weeks, either by further increase in size or additional signs of progression such as emergence of new lesions(117). Overall, to assess response to checkpoint inhibitors or immunotherapies in HCC, evaluation by CT/MRI at 812 weeks after treatment can be recommended, as opposed to the usual interval of 6-8 weeks for tyrosine kinase inhibitors. This window was used in phase II studies testing nivolumab (12 weeks)(38) and pembrolizumab (9 weeks)(116), where the phenomenon of pseudo-progression was reported as a marginal event.

\section{Design and endpoints for systemic therapies in HCC}

\section{Standard of care with systemic therapies in HCC}

Current estimates suggest that around $50 \%$ of HCC patients will receive effective systemic therapies during their lifespan $(3,119,120)$. Several trials have tried to show survival benefits of systemic agents in advanced disease (Table 2,4), a traditionally challenging setting due to the limited efficacy and high toxicity of conventional systemic chemotherapy(121-124). Randomized studies for anti-estrogen therapies also failed to prove any clinical efficacy (125). In 2008, the landmark SHARP trial assessing the multi-tyrosine kinase inhibitor sorafenib was the first to significantly improve survival with manageable adverse events(11). Afterwards, five treatments have succeeded, while several other drugs failed $(126,127),(22),(128)(129)(122)(130)(131)(132)(133)-$. In first line, atezolizumab (anti-PD-L1 inhibitor) plus bevacizumab (VEGFA inhibitor) have shown to be superior to sorafenib in a recently reported $\mathrm{RCT}(134)$. The study was stopped at the first interim analysis by showing a HR of 0.58 for OS (median not reached for combo vs 13.2 mo for sorafenib) and HR of 0.59 for PFS (median 6.8 mo for combo vs 4.3 for sorafenib). These results will pose this combination as standard of care first-line therapy for advanced HCC. Second, lenvatinib (multikinase inhibitor: VEGFRs, FGFRs, RET, KIT and PDGFRA) has become an option equal to sorafenib, after the positive result of the non-inferiority REFLECT study (HR of 0.92; $95 \% \mathrm{Cl}$ 0.79-1.06) (Table 2, Fig 2A). Because this trial excludes patients with main portal vein invasion, tumor involvement $>50 \%$ of the liver and clear bile duct invasion, the relative benefit of lenvatinib vs sorafenib in these patients remain uncertain. 
In second line, the phase III trial testing regorafenib (VEGFRs, PDGFRs, KIT and Tie2) improved OS compared to placebo from 7.8 to 10.6 months (HR of 0.63 ) in patients who progressed and were tolerant to sorafenib(8). The sequential treatment sorafenibregorafenib led to a median OS of 26 months compared to 19 months for sorafenib-placebo (23). These results need to be taken with caution since they will not apply to all patients receiving sorafenib, but only those able to receive the sequential treatment. The CELESTIAL study, showed median OS of 10.2 months with cabozantinib (VEGFRs, MET and $A X L)$ vs. 8 months with placebo (HR of 0.76$)(9)$; and the REACH-2 study, where ramucirumab (VEGFR2 monoclonal antibody) provided a median OS of 8.5 months in patients with AFP $\geq 400 \mathrm{ng} / \mathrm{ml}$ vs. 7.3 months with placebo (HR of 0.71$)(10,135)$. AFP is well-known for its independent prognostic capacity in $\mathrm{HCC}(136)$. As such, REACH-2 was the first and only positive phase III trial in a biomarker-driven population of patients with HCC (Fig 2B). In contrast, 3 phase III trials testing internal radiation with Y-90 for advanced HCC, either as single treatment [SARAH(137) and SIRveNIB(138)] or in combination Y-90 with sorafenib(139) did not meet the primary endpoint of improved OS compared to sorafenib (Fig. 2A). As a result, Y-90 was discouraged for the management of advanced HCC in the EASL guidelines (Fig. 1)(3). Despite appealing ORR of $15 \%$ with durable response for nivolumab and $18 \%$ for pembrolizumab, phase III trials comparing the former with sorafenib(24) in front-line and the latter with placebo in second-line resulted negative. Particularly, the latter trial showed a HR of 0.78 with upper boundary of $95 \mathrm{Cl}$ below 1 , but the pre-specified $p$ value $(p<0.0178)$ was not hit(41).

\section{Trial design in advanced HCC}

Overall survival remains as the primary end-point for advanced HCC research $(1,3)$ (Table 4). It has driven clinical research in HCC for more than 40 years and has been the goldstandard for measuring benefits at all stages of the disease. Nonetheless, the emergence of several effective drugs in advanced $\mathrm{HCC}$ has exposed the need for alternative end-points that can capture the benefits of a treatment before they can be diluted by post-progression therapy(3). Progression-free survival (PFS), time to progression (TTP) and objective response rate (ORR) are now emerging as tools to a) identify early strong signals of efficacy that led to accelerated regulatory approval (particularly ORR and PFS) $(6,88$ ) and b) test interventions which benefit can be assessed prior additional sequential drugs received beyond progression might mask the actual benefit of the tested drug. In this sense, a recent investigation analyzing 21 reported phase III studies(7-11,121-123,126$131,133,135,137,138,140$ ) in advanced HCC proposed PFS (with a restrictive hazard ratio criteria $<=0.6$ ) as a surrogate end-point for survival when testing kinase inhibitors or monoclonal antibodies, and thus as potential primary end-point in advanced HCC trials(3) (Table 4). Subsequently, six phase III studies have been released that confirm the hypothesis: two positive studies, one testing atezolizumab plus bevacizumab vs sorafenib(134) and the second sorafenib plus hepatic arterial infusion of Folfox vs sorafenib(141), both show HR for PFS $\leq 0.6$ and significant survival benefits, and four negative trials for survival testing nivolumab(24), sorafenib plus pravastatin(142), sorafenib plus doxorubicin(143) and pembrolizumab(41), in which the HR for PFS in all cases was $>0.6$ (Fig 3). Considering the special circumstances of the 2 negative trials testing anti-PD1 inhibitors, we should be cautious when applying this rule for testing immune therapies as single agents or for combinations of immune regimes.

Trial design in HCC has been evolving, and new challenges emerge as novel therapies become standard of care. Although there might be distinct approaches to trial design in $\mathrm{HCC}$, there has been a consensus on the basic principles that have been recently reported in guidelines and critical appraisals $(3,139,144,145)$. The key points are summarized below (Table 1): 
1. Phase II and Phase III trials: The panel recommends assessing drugs in the setting of randomized phase II studies before moving to phase III trials. Nonetheless, for some therapies, a large single arm phase II with a strong signal of efficacy might suffice to justify a phase III study. Thresholds for defining signals of efficacy are not clearly established, but for molecular therapies the ORR should likely be above $20-30 \%(146)$.

2. Selection of the target population: Clinical trials should consider BCLC staging system, Child-Pugh class and ECOG performance status for selection of the target population. In principle, for advanced HCC almost all RCT include patients with wellpreserved liver function (Child-Pugh A) and good performance status (ECOG 0 and 1).

3. Control arm: The control arm of randomized phase II and III studies should be the standard of care established according to guidelines. Although sorafenib and lenvatinib in front-line $(7,11)$ and regorafenib (8), cabozantinib(9) and ramucirumab (in patients with AFP $\geq 400 \mathrm{ng} / \mathrm{ml})(10)$ are accepted as standard of care, this will change when atezolizumab plus bevacizumab are approved by regulatory agencies. At that time, this combination will become the standard of care for comparison in front-line, and subsequence lines of therapy will move downwards. Double-blind trials (as opposed to open label trials) are recommended to prevent selection and allocation biases.

4. Stratification for prognostic factors prior to randomization: Stratification is critical in randomized studies to warrant balanced comparisons. For advanced $\mathrm{HCC}$ the recommendation is as follows: region, macrovascular invasion, extrahepatic spread, AFP > $400 \mathrm{ng} / \mathrm{ml}$ and ECOG 0 vs 1-2. Etiology should also be considered as studies with sorafenib and atezolizumab and bevacizumab suggest an influence of this factor in response.

5. End points: Overall survival: For systemic therapies the primary endpoint should be OS, and PFS is proposed as co-primary endpoint. To date, all regular FDA and EMA drug conventional approvals in advanced HCC were based upon improvements in OS. Surrogate endpoints: OS has limitations as a sole endpoint in cancer research: it might require a long follow-up to capture adequate numbers and can be affected by sequential therapies. Thus, surrogate endpoints that are more practical for trial execution are needed. There are no optimal surrogate endpoints able to recapitulate OS in HCC, and thus clinical practice guidelines do not recommend ORR, TTP and PFS as primary endpoints in phase III investigations(144,145). ORR is an independent predictor of OS in three phase II and III trials $(7,123,127)$, but is still considered a suboptimal primary end-point for phase III investigations. Nonetheless, ORR of $16-18 \%$ resulted in accelerated FDA approval of nivolumab and pembrolizumab in second line treatment of advanced $\mathrm{HCC}(38,39)$. PFS was formerly discarded as a primary end-point of phase III investigations due to the concept of competing risk of survival (competing between death due to tumor progression and due to the natural history of cirrhosis)(12). However, this competing risk drawback has been reduced by the universal selection of Child-Pugh $A$ patients for these investigations, thus reducing the $1-\mathrm{yr}$ risk of death due to decompensation to $<5 \%$. Stringent criteria for accepting PFS as primary endpoint have been proposed $(\mathrm{HR} \leq 0.6)$ and it is adopted in the current guidelines (Table 1), but this point is still controversial. Regarding ORR, use of both RECIST1.1. and mRECIST are proposed for the assessment of response in HCC treated with systemic therapy, whereas changes in serum biomarker levels (i.e. AFP levels) are not supported(3).

6. Magnitude of benefit: In HCC, there is no consensus on what absolute survival benefit (or magnitude of benefit in OS according to HR) is clinically relevant. Reported thresholds of OS with HR $<0.8$ are sound for capturing the benefit of patients in advanced HCC trials(20). This figure needs to be taken with caution, since other variables can impact 
the overall benefit of a given drug, such as quality of life, safety profile and availability of alternative therapies in distinct countries.

7. Checkpoint inhibitors and other immunotherapies have unique features and generally produce higher ORR and longer duration of response, as measured by RECIST1.1. The values of mRECIST and irRECIST in assessing checkpoint inhibitor mediated responses remain investigational.

\section{Immune treatments: Overview of results andspecific endpoints}

The initial clinical experience with checkpoint inhibitors in HCC was with a phase II study testing tremelimumab, a CTLA-4 antibody leading to objective response of $18 \%$ of patients and time to tumor progression was 6.5 months (147). Immunotherapy has drawn significant attention in HCC with the approval of nivolumab and pembrolizumab by the FDA based on promising results obtained in different phase II studies $(38,39)$. A phase I/II open-label, noncomparative trial (CheckMate 040) assessing the efficacy of nivolumab in advanced HCC reported objective response rate (ORR) of $20 \%$ in the dose-expansion phase $(n=214)$ and $15 \%$ in the dose-escalation phase $(n=48)$. Duration of response (DOR) was 9.9 months and median PFS as 4.0 months in the dose-expansion cohort. Nivolumab treatment was well tolerated (38). Pembrolizumab, another PD1 specific antibody, was tested in phase II in patients with HCC progressing or intolerant to sorafenib (Keynote 224). Pembrolizumab was effective and tolerable with one complete response (CR) and 17 PR out of 104 patients. The median progression free survival was 4.9 months, and median OS was 12.9 months(39). Camrelizumab, another fully humanized anti-PD-1 antibody, was evaluated in a randomized phase 2 trial in Chinese patients with advanced HCC after failure of at least one line of therapy(148). The ORR was $13.8 \%$ and the 6 -month OS was $74.7 \%$.

Nivolumab and pembrolizumab failed in phase III trials (Fig. 2A-B). Pembrolizumab was tested in a randomized, double-blind phase III trial against placebo in 443 patients with HCC that progressed on or were intolerant to sorafenib (Keynote-240). The co-primary endpoints of PFS and OS failed to reach the prespecified level of statistical significance although median OS was prolonged from 10.6 to 13.9 months (HR: $0.781 ; 95 \% \mathrm{Cl}$ : $0.611-0.998 ; \mathrm{P}=$ .0238)(149). Nivolumab was tested against sorafenib in a Phase 3 trial (CheckMate 459), but did not reach survival differences for superiority (24).In this RCT including around 750 patients, median OS for nivolumab was $16.4 \mathrm{mo}$ vs $14.7 \mathrm{mo}$ for the sorafenib arm (HR: 0.85 ; $95 \% \mathrm{Cl} 0.72-1.02)$. Objective response rate was $15 \%$ and $7 \%$, respectively.

Anti-CTLA4 antibodies have been tested as single agent(147) or in combination with locoregional therapies(150) and are under investigation in combination with anti-PD1 drugs(151). In this regard, very recently the combination ipilimumab and nivolumab received FDA approval based on a ORR of $31 \%$ (40). Currently phase III trials are ongoing which either test the combination of two immune checkpoint inhibitors, immune checkpoint inhibitors plus TACE, immune checkpoint blockade in the adjuvant setting or immune checkpoint inhibitors plus vascular targeting agents(152). While the overall response to immune checkpoint inhibition (15-20\%) may not be as dramatic as initially hoped, complete responses are seen in a small number of cases in almost every trial. This observation together with the recent results from two phase III trial testing anti-PD1 antibodies in the first- and second-line setting rise up the important question of what endpoint to use in future trials. While OS remains the "gold standard" it should be noted that HCC is not the only cancer where this question is being asked. Due to the unique mechanism of action of immune checkpoint inhibitors(153), new endpoints such as ORR and surrogate biomarkers have been tested and new immune related RECIST criteria devised to capture distinctive 
patterns and timing of response to immunotherapy $(35,115)$. Finally, while immunological endpoints may be helpful as surrogates(154), they cannot be recommended at this time.

A systematic review and meta-analysis of 87 phase 2 trials with the foal of defining the most appropriate primary endpoint in phase 2 trials of immune checkpoint inhibitors for advanced solid cancers has been reported. Correlations between ORR odds ratios and hazard ratios (HRs) for PFS and OS were examined for randomized comparisons. Within checkpointinhibitor treatment arms, correlations of ORR with 6-month PFS and 12-month OS rates were examined. All analyses were weighted by trial size. Multivariable models to predict 6month PFS and 12-month OS rates from ORR were developed and their performance validated in an independent sample of trials. The authors demonstrated that ORR correlated poorly with OS and recommended 6-month PFS rate as an endpoint for future phase 2 studies,(155). Thus, PFS endpoint can also be recommended for studies evaluating immune checkpoint inhibitors in HCC (Table 1).

\section{Biomarker-Driven Trials in HCC: Results and Endpoints}

Recent clinical trials in advanced HCC are demonstrating that the sequential use of systemic agents is changing the natural history of the disease. Still, these results are incremental and the incorporation of biomarker driven strategies have generally been unsuccessful. Unlike other solid tumors such as breast, lung, colon cancer, and others, where therapeutic decisions are driven by an understanding of a patient given molecular features, in HCC a "one-size-fits-all approach" is still the usual approach to patients. This applies to all therapies so far accepted in guidelines, except for ramucirumab.

Biomarkers provide the distinct possibility of supplementing existing anatomic and/or pathologic information to provide a more accurate assessment of prognosis (to be used for patient stratification) and/or to identify individuals who are more likely to respond to specific therapy (predictive of response) (156-158) There is a plethora of literature on the different predictive biomarker validation designs (159). The $\mathrm{NCl}$ defines a biomarker as a biological molecule found in blood, other body fluids, or tissues that is a sign of a normal or abnormal process, or of a condition or disease. A biomarker may be used to determine how well the body responds to a treatment for a disease or condition (160).

In HCC, numerous studies have defined the molecular heterogeneity of the disease and specific genetic alterations and subtypes. These data are fertile ground for testing biomarker hypotheses as both prognostic and/ or predictive markers in prospective studies but so far these data have largely been ignored in clinical development in HCC (6). To date, two phase 3 studies have tested biomarker driven approaches. Firstly, tivantinib, a small molecule inhibitor of the hepatocyte growth factor/ c-MET was evaluated in patients that had progressed on sorafenib and had elevated expression of c-MET in their tissue. This was a placebo-controlled study that yielded negative results(131). The possible reasons for failure highlight the challenges with this approach including: 1) validity of the target, 2) robustness of the assay for patient selection, and 3) ability of the agent to inhibit the target successfully in tumor tissue. The latter may be a plausible reason for failure of the trial, considering that the anti-MET activity of this drug has been challenged in experimental studies(161). Conversely, ramucirumab, which initially failed in an "all comers" study(162), demonstrated an improvement in OS for selected patients with AFP $\geq 400 \mathrm{ng} / \mathrm{ml}$. Proof-of-concept studies testing small molecule inhibitors of FGFR-4 using biomarker enriched populations based on FGF-19 expression have been reported with ORR of 16\% (163).

Recently, immunotherapy approaches have garnered high interest in the management of HCC and the PD-1 directed antibodies nivolumab and pembrolizumab received accelerated approval by the US FDA $(38,39)$. However, unlike in other cancers, PD-1 and / or PD-L1 
expression has not correlated with outcome. This has likely contributed to the recent negative results from phase 3 studies with these agents(149). Ongoing work is focused on further refining biomarker development evaluating other inflammatory markers including incorporation of more broad based assessment tools such as an immune-enriched signature identified through molecular profiling of HCC (164).

Several studies have incorporated biomarker assessments into the trial design. While tissue collection is often optional and therefore limited, serum assays have served to generate hypotheses for further study. In the pivotal SHARP study, baseline levels of angiopoietin 2 and VEGF were prognostic but not predictive of benefit from sorafenib(136). Relevant biomarkers in the FGF and VEGF pathways were analyzed in the REFLECT study and identified differences in the modulation of these pathways by lenvatinib and sorafenib, but no biomarker could define a group receiving differential benefit from either compound(165). In the REACH-2 study, decreases in AFP correlated with better outcome from ramucirumab(10). Novel study designs evaluating biomarker assessments pre-and posttreatment are being performed. These so-called "pre-surgical" studies are designed to acquire tissue at baseline, from patients with resectable tumors, expose the patient to a novel agent for a short period, and then collect tissue at the time of resection. Molecular studies comparing the pre-and post-treatment tissue provide an opportunity to understand the effects of novel therapeutics on relevant pathways in the tumor. These studies can provide critical information that could guide a patient selection strategy in conventional efficacy studies. One such study with nivolumab is producing interesting insights into tumor characteristics that may correlate to response to this drug(166).

Despite the recent successes in clinical trials of new agents for HCC, the improvements in survival are modest. Throughout cancer medicine, the largest impacts in outcomes have been by biomarker driven drug development. Examples include ALK(167) and EGFR(168) testing in lung cancer, HER-2(169) and estrogen receptor(170) testing in breast cancer, c$\mathrm{KIT}$ testing in gastrointestinal tumors(171), and BCR-ABL testing in chronic myelogenous leukemia(172). By enriching for the population most likely to benefit, studies can be conducted with smaller numbers of patients and minimize risk for failure. While historically predictive marker testing is done on tumor tissue obtained by biopsy, newer technologies are now allowing biomarker detection in peripheral blood. The practice of not obtaining biopsies for diagnosis of HCC, the fact that most common driver mutations in HCC are nonactionable and the observation that only $25 \%$ of HCCs harbor at least one actionable mutation(172), in contrast to the majority of solid tumors(173), have hindered development of biomarker driven precision treatment to date. Nonetheless, there is now renewed interest in incorporating tissue acquisition into clinical trials, not only in the early part of drug development, but in later studies as well. Clinical trial designs for predictive marker validation are inherently complex and require data from a randomized controlled clinical trial $(\mathrm{RCT})(153)$. There is a plethora of literature on the different predictive biomarker validation designs, including articles that specifically focus on the statistical and clinical properties and assumptions of these different trial designs (156).

Trial design in the precision medicine era require a platform for biomarker profiling (173)(174). The ultimate clinical utility of a biomarker will depend on: 1) its added value in every patient in the context of the markers prevalence, 2) its incremental benefit for treatment selection when considering the added costs and complexity induced by the use of the marker, and 3) the added effectiveness of the new treatment option in all patients versus biomarker-defined subgroups. 


\section{Liquid biopsy in early HCC detection, prediction of response and tumor relapse}

Liquid biopsy entails the analysis of tumor components released by cancer cells to biological fluids such as blood, saliva or cerebrospinal fluid(175). The concept includes the analysis of actual cancer cells (i.e., circulating tumor cells [CTCs]), fragments of DNA from necrotic or apoptotic cancer cells (i.e., circulating tumor DNA [ctDNA]) and extracellular vesicles(176). Compared to conventional tissue biopsies, the main advantages of liquid biopsy using samples from peripheral blood are: 1) it is minimally invasive, which eliminates the complications associated with invasive tissue biopsies; 2) it facilitates sequential sampling, which is crucial to better select therapies in patients receiving multiple lines of treatment; 3) it enables tracking tumor clonal composition in heterogeneous tumors, a feature that allows earlier detection mechanisms of treatment resistance; and 4) it can be implemented as a point-of-care diagnostic. Potential clinical applications include cancer surveillance, early detection of minimal residual disease after curative therapies, prognostic prediction and molecular monitoring of therapeutic response(177). In an early sign of impact on patient care, the FDA recently approved the use of a ctDNA-based test to detect mutations of EGFR in lung cancer patients who are candidates to receive EGFR-based tyrosine kinase inhibitors(178).

In HCC, liquid biopsy has been evaluated for three clinical applications: early HCC detection in the context of surveillance, as a prognostic biomarker after surgical resection and to predict response to systemic therapies. Mutation profiling of ctDNA is feasible and confidently detects tissue mutations in early stage $\mathrm{HCC}(179)$. A recent report combining data from ctDNA and protein markers had a sensitivity and specificity of $85 \%$ and $93 \%$ respectively for the detection of $\mathrm{HCC}(180)$. Also, analysis of DNA methylation alterations in ctDNA has high accuracy for HCC diagnosis $(55,181)$. A study that included a gene signature derived from CTCs was able to accurately discriminate between HCC patients and controls(182). Higher CTC count correlates with a greater risk of tumor recurrence after surgical resection(175). There are few studies using liquid biopsy to predict response to systemic therapies in HCC. A retrospective study suggested that HCC patients with ctDNA detectable VEGFA DNA amplifications have better outcomes when treated with sorafenib(183). Also, RAS mutations analysis of ctDNA was used to determine eligibility to receive refametinib in a phase 2 clinical trial(184). Thus, there is increasing interest in applying this technology to predict response to systemic therapies.

\section{Quality of life and patient reported outcomes}

Systematic capture of the patient perspective can inform the development of new cancer therapies. The U.S. FDA Office of Hematology and Oncology Products (OHOP) has identified symptomatic adverse events (AEs) as a central Patient Reported Outcome (PRO) using the National Cancer Institute's Patient-Reported Outcomes version of the Common Terminology Criteria for Adverse Events (PRO-CTCAE) to provide a standard yet flexible method to assess symptomatic AEs from the patient perspective $(194,195)$. The FDA's patient focused drug development program has ongoing efforts to improve methods around the collection, analysis and interpretation of PRO data, as well as initiatives to identify patient-friendly language, and leveraging digital health tools. In 2016, the 21st Century Cures Act tasked the FDA to consider the patient-experience in the risk-benefit determination. The FDA draft guidance outlines the use of PRO to assess symptomatic side effects and the core set of clinical outcomes to measure in cancer trials, including design considerations and assessment frequency(11).

The purpose of measuring quality of life (QOL) should be to compare outcomes between treatment arms, even if one is a placebo. There are two methods of measuring QOL specific to HCC: the European Organization for Research and Treatment of Cancer Quality of Live 
Questionnaire (EORTC QLQ)(185) and the Functional Assessment of Cancer TherapyHepatobiliary (FACT-Hep)(186) questionnaire. Few studies have adequately assessed PRO using these tools in HCC research, a recommendation that is endorsed by the panel.

Most phase III trials for HCC were designed primarily to compare two different treatments in patients with similar stage disease. For example, one study compared QOL after resection with QOL following radiofrequency ablation(187). As expected, QOL was much better after radiofrequency ablation than after resection, and remained superior up to 36 months post-treatment. In addition, QOL following radioembolization has been compared with TACE(188). In this study, there was no overall difference in QOL between the two groups, but the sample size was small. Despite the lack of statistically significant differences, in the TACE group QOL was decreased at 2 and 4 weeks, whereas in the radioembolization group some aspects of QOL actually improved. Similarly, QOL measures favoring Y-90 vs sorafenib have been claimed to support the former treatment in three negative RCT, the SARAH trial (134) and the SIRveNIB trial (135) and SORAMIC trials. However, since indication of a drug/device should be based upon the primary endpoint (survival), no actual indication can be claimed if the result is negative for the primary endpoint. Finally, the SHARP trial demonstrating survival benefit of sorafenib also tested timeto-symptomatic progression - as measured by the Functional Assessment of Cancer Therapy-Hepatobiliary Symptom Index 8 (FHSI8) - as a co-primary endpoint. The negative results of this end-point contrasted with the survival benefit obtained by sorafenib, thus challenging the accuracy of the tool used(11). More recently, patient reported outcomes have been tested in the setting of phase III investigations showing significant results in positive RCT in advanced HCC. This is the case of lenvatinib compared to sorafenib, or atezolizumab plus bevacizumab compared to sorafenib, where the tested arms showed better QoL parameters compared with the standard of care. The panel encourage the integration of these endpoints in all investigations in HCC (Table 6).

\section{Implications of trial design in Asia}

\section{Differences between AASLD, EASL, and Asian guidelines}

Recommendations in western guidelines (AASLD and EASL) are based upon evidence from clinical trials (Table 4), while Asian guidelines integrate evidence with expert consensus and clinical practice. Applicability of those guidelines varies according to region and treatment stage (47). Asian guidelines (189) in general recommend ablation or resection for very early-stage (stage 0 ) disease, but differ from western guidelines in the recommendations at other stages of disease. For instance, TACE or yttrium-90 (Y90-SIRT) are recommended for single large tumors, and systemic therapies -i.e Folfox(105), or hepatic arterial infusion chemotherapy - are recommended for advanced stages, along with liver transplantation - mostly living donor transplantation. Similarly, in Asia patients with portal vein invasion and well-preserved liver function might be considered for TACE, resection or radiotherapy (190-194).

\section{Specificities of trial design in Asia}

Considering all these guidelines, trial design in Asia has some specificities. For instance, resection in very high-risk patients (multinodular tumors, macrovascular invasion) is common in Asia, and thus adjuvant trials might consider this indication with a recurrencefree survival endpoint. Similarly, studies exploring the role of systemic therapies plus TACE in patients with advanced stages might also be considered in Asia with a primary end point of PFS. Weather these approaches should be tested in specific trials or as part of global trials needs further consideration. 


\section{Future prospects}

\section{The dawn of a new era: combination therapies}

When the report of the first AASLD conference on Design and Endpoints of Clinical Trials in HCC was published(12), the field was still heady with excitement from the first positive trial of a systemic agent for advanced HCC, which established sorafenib as the first FDA approved systemic therapy for HCC (11). However, enthusiasm was also tempered by the subsequent negative results of trials of sorafenib as adjuvant therapy after resection or ablation (STORM)(76) or in combination with TACE (SPACE)(94). It was recognized then that a unique challenge is posed by the combination of underlying chronic liver disease with a very heterogeneous and variably aggressive primary HCC. It is therefore important that treatment strategies account for both the liver disease and malignancy, and thus variables capturing both diseases should be considered in the publication of clinical trials for HCC (Table 7). Discussions at the previous AASLD Endpoints conference set the framework for subsequent attempts to bring additional agents to approval, which were met with uniformly disappointing results over the next several years, with trial failures due to unacceptable toxicity or inadequate efficacy(12). While disappointing, these failures led to robust examination of the optimal approach to trial design and catalyzed a more rigorous approach that contributed to the successes in phase $3 \mathrm{HCC}$ clinical trials. With the positive results and approvals of lenvatinib, regorafenib, cabozantinib and ramucirumab based on phase 3 studies, and of the checkpoint inhibitors nivolumab and pembrolizumab based on convincing phase 2 data, we appear to be poised for success in the next most logical treatment paradigms using combination therapies. Indeed, the recent positive phase III study demonstrating superior OS for atezolizumab plus bevacizumab vs sorafenib(134), represents the dawn of a new era of combination therapies in all stages of HCC research (Fig 1). This combination is certainly first in-class of this approach. Whether other combinations may become best-in-class will depend upon the ability of specific TKI and/or MAb to transform "cold tumors", which are primary resistant to immunotherapy, into "hotimmune-active tumors", allowing checkpoint inhibitors to optimally unleash immune attack against cancer cells(195-197) .

The advent of combination therapies achieving response rates of $30 \%$ and survival rates above 20 months in advanced HCC provides the rationale for testing these combinations in earlier HCC stages. Currently, phase III trials testing combination therapies are ongoing for early stages (neo-adjuvant or adjuvant approaches), intermediate $\mathrm{HCC}$ (in combination with TACE or in comparison to TACE) and in front-line trials for advanced HCC (Table 7). It is conceivable that systemic therapies may be incorporated in all areas of HCC management in the near future. Thus, the up-dated target population and endpoints described here should be valuable in this endeavor.

\section{Understanding tumor biology remains critical: tissue and blood samples are needed}

It is likely that the next key advances in HCC therapy will emanate from an improved understanding of HCC biology and the ability to predict the response of specific HCCs subgroups to particular therapies. Until now, most HCC therapy has been applied in a biologically indiscriminate fashion. The biological heterogeneity of HCC has been evident for many years, demonstrated by differences in phenotypes, tumor growth rates, numbers of tumor nodules, discrete versus infiltrative appearance, propensity for microvascular or macrovascular invasion, propensity for distant metastasis, and association with elevation of AFP, AFP-L3, DCP and other biomarkers. Apart from the limitations that multifocal, invasive or metastatic disease have placed on application of potentially curative treatments such as surgical resection, liver transplantation, and local ablation, we have only recently began to incorporate markers of tumor biology into therapeutic decision making. Applications of tumor 
biologic characteristics into therapeutic approaches have been scarce in HCC and mostly focused on using AFP levels for selection policy for transplantation, as an stratification factor in most of trials and finally for selecting candidates to ramucirumab in the management of advanced HCCs in second line.

With the advent of next generation DNA, RNA and non-coding RNA sequencing and similar genome wide methodologies for copy number variation, methylation and proteomic characterization, we now stand ready to translate information from these technologies to the care of HCC patients, transforming the selection of systemic therapy and the selection of optimal candidates for loco-regional therapies. Results suggesting that CTNNB1-mutated HCCs are immune excluded and potentially resistant to immune checkpoint inhibitors(198200 ) but potentially susceptible to mTOR inhibitors are an early indication of the potential value of genomics in personalizing HCC therapy. These studies may also provide us with tools for better understanding the recent borderline negative results of phase 3 trials with single agent immune checkpoint inhibitors. Personalization of therapy using molecular and genomic signatures will require integration of molecular subclasses into clinical staging systems, to better guide treatment selection. Optimal treatment selection will depend on the ability to target oncogenic signaling pathways that drive tumorigenesis, tumor progression and metastasis. The development of new preclinical tumor models, including organoids, patient derived xenografts, and syngeneic models that preserve aspects of the immune response will be critical for the testing of novel agents and combinations. Ideally, integration of molecular profiling into the HCC treatment paradigm will require genomic data derived in real time from patients, either by tissue biopsy or through liquid biopsy-based access to circulating tumor DNA or other analytes. This will require a cultural change in the care of HCC patients, shifting from a state in which the diagnosis and evaluation of patients is performed non-invasively, to regular use of tissue biopsy and highly sensitive liquid biopsy assays. Development of robust, reproducible predictive biomarkers of high reliability is a key priority to facilitate this transition (Table 6). The first implementations of the biomarkerbased approaches should be within RCT, which should now routinely require tissue biopsy and liquid biopsy collection as a condition of trial enrollment. Tumor biopsy at screening for trial entry and liquid biopsy at different time points should be mandatory in clinical trials for advanced HCC to allow identification of prognostic and predictive biomarkers, guide clinical decision-making and improve patient outcomes.

\section{Novel endpoints might be adopted}

The revolution in drug development in HCC has created the need to revisit established conventions in trial design. OS is regarded as a core endpoint. Nonetheless, the realization that more than $60 \%$ of patients progressing after TACE and $50 \%$ of patients progressing after first-line systemic therapies receive effective next line therapies may compel the adoption of PFS as an acceptable primary endpoint for major trials (Table 1). In this position paper, we already are recommending PFS as co-primary endpoint for intermediate HCC trials, and for phase II-III trials assessing systemic therapies, with restrictive cut-points. Similarly, patient reported outcomes (PRO) should be pursued as a relevant endpoint in HCC trials, particularly as we enter an era of potent, but seemingly toxic, dual or triple combination therapies possibly associated with serious adverse events. It is important to recognize that currently many HCC patients reach a point in their therapeutic journey when they elect to forgo potentially life extending therapy in favor of approaches that optimize their quality of life. It is therefore critical to extend decisions about HCC trial design and endpoints to incorporate elements that reflect the importance of patient well-being. 


\section{FIGURE LEGENDS}

Figure 1. Modified BCLC staging system considering new effective therapies in advanced stages [modified and up-dated from EASL Guidelines(3)] Management of patients with HCC is guided by the Barcelona Clinic Liver Cancer (BCLC) staging system, which takes into account both tumor extent and the severity of the underlying liver disease, and defines five prognostic subgroups with respective treatments. Treatment for early stage tumors is with curative intent and options include radiofrequency ablation, hepatic resection, and liver transplantation. Patients with intermediate or advanced HCC are candidates for chemoembolization or systemic therapies, respectively. ${ }^{*}$ Patients with end-stage liver disease if Child-Pugh class $C$ should first be considered for liver transplantation. ${ }^{* *}$ Patients with preserved hepatic function Child-Pugh class $A$ with normal bilirubin and no portal hypertension are optimal candidates for hepatic resection. ¥The combination of atezolizumab plus bevacizumab is not yet approved but is set to become the new first-line treatment for advanced HCC(134)- DDLT, deceased donor liver transplantation; ECOG, Eastern Cooperative Oncology Group; LDLT, living donor liver transplantation; M1, distant metastasis; N1, lymph node metastasis; OS, overall survival.

Figure 2. Overall survival outcomes (HR, 95\% Cl) of phase III clinical trials testing molecular targeted therapies, checkpoint inhibitors and radioembolization in patients with advanced-stage hepatocellular carcinoma. Blue=positive trials for superiority. Orange=positive trials for non-inferiority. Black=negative trials for the primary end-point, Red=tested drug was significantly worse than the standard of care. Vertical red line at $\mathrm{HR}=1.08$ defines the upper boundary of $95 \%$ confidence interval accepted by FDA for a positive non-inferior study.

Figure 3. Correlation between progression-free survival and overall survival in 27 phase III trials of advanced HCC (modified from Llovet, J Hep 2019(20)) . Trial-level correlation between endpoints using criteria from the Institute for Quality and Efficiency in Health Care (IQWIG). R and R2 refers to the weighted Pearson coefficient between the HR of OS and the HR of the surrogate endpoint. Each dot represents one of the phase III clinical trials conducted on advanced HCC. Size of the dot is proportional to the total number of patients enrolled in the trial. First 21 phase III trials defined a cut-off of 0.6 for PFS to correlate with a significant OS (colored in grey)(20). Afterwards, six additional phase III trials have been reported afterwards: two positive for survival show a HR for PFS <0.6 (green color) and four negative for OS show a PFS HR> 0.6 (red color). X-and Y-axis depict the value of the HR for the surrogate and the hard endpoint, respectively. Gray shaded areas represent the upper and lower limits of the 95\% confidence intervals for the regression. $\mathrm{HCC}$, hepatocellular carcinoma; HR, hazard ratio; OS, overall survival. 


\section{TABLES}

Table 1. Recommendations for Trial design and endpoints in patients with HCC by AASLD panel of experts.

\begin{tabular}{|c|c|c|}
\hline Aim & Factor & $\begin{array}{l}\text { Considerations } \\
\text { recommendations }\end{array}$ \\
\hline \multirow[t]{3}{*}{$\begin{array}{l}\text { Select the target } \\
\text { population }\end{array}$} & BCLC stage & $\begin{array}{l}\text { Include patients according to } \\
\text { specific BCLC stage }(0-C)\end{array}$ \\
\hline & Child-Pugh classification & $\begin{array}{l}\text { Include patients in Child-Pugh } \\
\text { class A. } \\
\text { Consider ALBI grade and MELD } \\
\text { score for refinements on Child A } \\
\text { class }\end{array}$ \\
\hline & $\begin{array}{l}\text { Biomarker-based } \\
\text { enrichment }\end{array}$ & $\begin{array}{l}\text { Define rationale for using } \\
\text { biomarker and tool }\end{array}$ \\
\hline \multirow[t]{3}{*}{ Selection of endpoints } & Overall survival (OS) & $\begin{array}{l}\text { Primary endpoint for phase II and } \\
\text { III studies assessing treatments } \\
\text { in intermediate and advanced } \\
\text { HCC. }\end{array}$ \\
\hline & $\begin{array}{l}\text { Progression-free survival } \\
\text { (PFS) }\end{array}$ & $\begin{array}{l}\text { Primary endpoint for Phase II } \\
\text { studies assessing primary } \\
\text { treatments in intermediate and } \\
\text { advanced HCC. } \\
\text { Consider co-primary in phase III } \\
\text { studies in intermediate and } \\
\text { advanced HCC, with strict rules } \\
\text { for calling superiority } \\
\text { Independent centralized blinded } \\
\text { review* }\end{array}$ \\
\hline & $\begin{array}{l}\text { Time to Progression } \\
\text { (TTP) }\end{array}$ & $\begin{array}{l}\text { Secondary (or co-primary) } \\
\text { endpoint for Phase II studies } \\
\text { assessing primary treatments in } \\
\text { intermediate and advanced } \\
\text { HCC. } \\
\text { Independent centralized blinded } \\
\text { review* }^{*}\end{array}$ \\
\hline
\end{tabular}




\begin{tabular}{|c|c|c|}
\hline & $\begin{array}{l}\text { Recurrence-free survival } \\
\text { (RFS) }\end{array}$ & $\begin{array}{l}\text { Primary endpoint in phase II/III } \\
\text { studies assessing adjuvant } \\
\text { treatments }\end{array}$ \\
\hline & $\begin{array}{l}\text { Time to recurrence } \\
(\mathrm{TTR})\end{array}$ & $\begin{array}{l}\text { Primary endpoint }\left(2^{\text {nd }} \text { choice }\right) \text { in } \\
\text { phase II/II studies assessing } \\
\text { adjuvant treatments }\end{array}$ \\
\hline & Objective response rate & $\begin{array}{l}\text { Phase II co-primary endpoint, } \\
\text { Phase III secondary end-point }\end{array}$ \\
\hline & & $\begin{array}{l}\text { Surrogate endpoint for } \\
\text { accelerated approval }\end{array}$ \\
\hline & & $\begin{array}{lcr}\text { Independent } & \text { blinded review } \\
\text { assessing } & \text { mRECIST } & \text { for } \\
\text { interventions } & \text { at } & \text { early/ } \\
\text { intermediate } & \text { HCC. Both RECIST } \\
1.1 \text { and } & \text { mRECIST for } \\
\text { interventions at advanced stages }\end{array}$ \\
\hline & $\begin{array}{l}\text { Patient reported } \\
\text { outcomes (PRO) }\end{array}$ & $\begin{array}{l}\text { Recommended as secondary } \\
\text { endpoint in all phase III } \\
\text { investigations, particularly when } \\
\text { testing loco-regional or systemic } \\
\text { therapies }\end{array}$ \\
\hline & Composite endpoints & $\begin{array}{l}\text { OS plus PFS might be } \\
\text { considered }\end{array}$ \\
\hline Select control arm & Surveillance & Ultrasound with or without AFP \\
\hline & $\begin{array}{l}\text { Adjuvant therapy } \text { after } \\
\text { resection or } \\
\text { ablation for early } \\
\text { HCC }\end{array}$ & Placebo \\
\hline & Early stage-non surgical & $\begin{array}{l}\text { Radiofrequency ablation. Special } \\
\text { consideration for single large } \\
(>4 \mathrm{~cm} \text { tumoral diameter) when } \\
\text { standard of care is TACE as per } \\
\text { the stage migration principle. }\end{array}$ \\
\hline & $\begin{array}{l}\text { Intermediate stage } \\
\text { disease }\end{array}$ & Chemoembolization** \\
\hline & $\begin{array}{l}\text { First line treatment for } \\
\text { advanced stage disease }\end{array}$ & $\begin{array}{l}\text { Atezolizumab plus bevacizumab } \\
\text { will be considered when }\end{array}$ \\
\hline
\end{tabular}




\begin{tabular}{|c|c|c|}
\hline & & $\begin{array}{l}\text { approved. Sorafenib or lenvatinib } \\
\text { plus supportive care } \\
\text { Other treatments in Asia** }\end{array}$ \\
\hline & $\begin{array}{l}\text { Second-line treatment } \\
\text { for advanced stage } \\
\text { disease }\end{array}$ & $\begin{array}{l}\text { Regorafenib (only in patients } \\
\text { tolerant to sorafenib) or } \\
\text { cabozantinib. Ramucirumab only } \\
\text { in patients with AFP> } 400 \mathrm{ng} / \mathrm{ml}\end{array}$ \\
\hline & $\begin{array}{l}\text { Third-line treatment for } \\
\text { advanced stage disease }\end{array}$ & Placebo \\
\hline \multirow[t]{4}{*}{$\begin{array}{l}\text { To stratify factors before } \\
\text { randomization }\end{array}$} & Adjuvant & $\begin{array}{l}\text { A) Geographical region } \\
\text { B) Tumor size and number } \\
\text { C) Type of curative treatment } \\
\text { D) Pathological factors of high } \\
\text { risk (size >3cm, microvascular } \\
\text { invasion, poor differentiation } \\
\text { degree and tumor satellites) }\end{array}$ \\
\hline & Intermediate-stage & $\begin{array}{l}\text { Child-Pugh class, AFP >400 ng/ } \\
\text { ml and geographical region. ALBI } \\
\text { score might be considered. } \\
\text { Selection of large tumoral burden } \\
\text { as per above } 7 \text {-up-to- } 7 \text { has been } \\
\text { proposed (adopting criteria for } \\
\text { extended indications in liver } \\
\text { transplantation), but requires } \\
\text { validation }\end{array}$ \\
\hline & First-line advanced stage & $\begin{array}{l}\text { ECOG status, MVI-EHS, AFP } \\
>400 \mathrm{ng} / \mathrm{ml} \text { and geographical } \\
\text { region, Etiology (HCV vs others } \\
\text { when testing sorafenib) }\end{array}$ \\
\hline & $\begin{array}{l}\text { Second-line advanced } \\
\text { stage }\end{array}$ & $\begin{array}{l}\text { ECOG status, MVI, EHS, } \\
\text { geographical region, AFP }>400 \\
\text { ng/ ml. Type of progression might } \\
\text { be considered }\end{array}$ \\
\hline
\end{tabular}


AFP, a-fetoprotein; BCLC, Barcelona Clinic Liver Cancer; ECOG, Eastern Cooperative Oncology Group; HCC; hepatocellular carcinoma; EHS=extrahepatic spread; MVI=macrovascular invasion; RECIST, Response Evaluation Criteria In Solid Tumours.

* $=$ not always recommended

$* *=$ Asian guidelines recommend additional treatments for

Intermediate HCC: Japan: HAIC; China: Resection; Taiwan: Resection/Y90

Advanced HCC: Japan : HAIC/Resection/TACE; China: FOLFOX4, resection/TACE; Korea: TACE 
Table 2. Expected outcomes reported in phase III trials in HCC research.

\begin{tabular}{|c|c|c|c|c|}
\hline $\begin{array}{l}\text { Expected } \\
\text { outcomes }\end{array}$ & Early & Intermediate & $\begin{array}{l}\text { Advanced } \\
\left(1^{\text {st }} \text { line }\right)^{* * *}\end{array}$ & $\begin{array}{l}\text { Advanced } \\
\left(2^{\text {nd }} \text { line }\right)\end{array}$ \\
\hline $\begin{array}{l}\text { Overall } \\
\text { survival }\end{array}$ & & $\begin{array}{lr}\text { TACE: } & 21 \\
26 \mathrm{mo}(93) \\
\mathrm{mo}(96) & (95)-33 \\
\end{array}$ & $\begin{array}{l}\text { Sorafenib: 11-14(7,11,24) } \\
\text { mo } \\
\text { Lenvatinib: } \sim 13 \mathrm{mo}(7) \\
\begin{array}{l}\text { Atezolizumab+bevacizumab } \\
* * *(134)\end{array}\end{array}$ & $\begin{array}{l}\text { Regorafenib: } \sim 11 \mathrm{mo}(8) \\
\text { Cabozantinib: } \sim 10 \mathrm{mo}(9) \\
\text { Ramucirumab (only AFP> } \\
400 \mathrm{ng} / \mathrm{ml} \text { ): } \sim 8 \mathrm{mo}(10)\end{array}$ \\
\hline PFS & $\begin{array}{l}\text { RFS*adjuvant } \\
\text { resection } \\
\text { /ablation: } 33 \mathrm{mo}\end{array}$ & TACE: $7 \mathrm{mo}(93,95)$ & $\begin{array}{l}\text { Sorafenib: } \sim 4(7) \text { months } \\
\text { Lenvatinib: } \sim 7 \text { months }(7)\end{array}$ & $\begin{array}{l}\text { Regorafenib: } \sim 3 \mathrm{mo}(8) \\
\text { Cabozantinib: } \sim 5 \mathrm{mo}(9) \\
\text { Ramucirumab (only AFP> } \\
400 \mathrm{ng} / \mathrm{ml}): \sim 3 \mathrm{mo}(10)\end{array}$ \\
\hline $\mathrm{ORR}^{*}$ & & $\begin{array}{l}\text { TACE: } \quad \sim 45-54 \% \\
(93)\end{array}$ & $\begin{array}{l}\text { Sorafenib: } \sim 10 \%(7) \\
\text { Lenvatinib: } \sim 24 \%(7) \\
\text { Atezolizumab+bevacizumab } \\
: 33 \% \text {; RECIST: } 27 \%(134)\end{array}$ & $\begin{array}{l}\text { Regorafenib: } \sim 10 \%(8) \\
\text { Cabozantinib: } \sim 4 \%(9) \\
\text { Ramucirumab (only AFP> } \\
400 \mathrm{ng} / \mathrm{ml} \text { ): } \sim 5 \%(10)\end{array}$ \\
\hline
\end{tabular}

${ }^{*}$ RFS: Recurrence free survival

** ORR as per mRECIST

***Atezolizumab+bevacizumab is expected to be fist line, while sorafenib and lenvatinib will be second line therapies, see Fig 1 
Table 3. Phases of Surveillance Test Validation in Hepatocellular Carcinoma [(adopted from Pepe et al(57))].

\begin{tabular}{|c|c|c|c|c|}
\hline Phases & $\begin{array}{ll}\text { Type } & \text { of } \\
\text { Study } & \end{array}$ & Design & Aims & Comments \\
\hline 1 & $\begin{array}{l}\text { Preclinical } \\
\text { exploratory }\end{array}$ & $\begin{array}{l}\text { Case-control from } \\
\text { biobanked } \\
\text { samples }\end{array}$ & $\begin{array}{l}\text { Promising } \quad \mathrm{HCC} \\
\text { biomarkers } \\
\text { identified }\end{array}$ & $\begin{array}{lr}\text { Avoid multiple } \\
\text { freeze-thaw for } \\
\text { blood and tissue } \\
\text { samples }\end{array}$ \\
\hline 2 & $\begin{array}{l}\text { Clinical Assay } \\
\text { and } \\
\text { Validation }\end{array}$ & $\begin{array}{l}\text { Large case- } \\
\text { controlled } \\
\text { accounting for } \\
\text { confounders }\end{array}$ & $\begin{array}{lr}\text { Biomarker } & \text { detects } \\
\text { clinically } & \\
\text { established } & \text { early } \\
\text { stage HCC } & \end{array}$ & $\begin{array}{l}\text { Appropriate sample } \\
\text { size and power } \\
\text { essential }\end{array}$ \\
\hline 3 & $\begin{array}{l}\text { Retrospective } \\
\text { longitudinal }\end{array}$ & PRoBE & $\begin{array}{l}\text { Biomarker detects } \\
\text { pre-clinical HCC }\end{array}$ & $\begin{array}{l}\text { Assess benefits } \\
\text { and harms of } \\
\text { surveillance }\end{array}$ \\
\hline 4 & $\begin{array}{l}\text { Prospective } \\
\text { screening }\end{array}$ & $\begin{array}{l}\text { Prospective } \\
\text { cirrhosis cohort }\end{array}$ & $\begin{array}{l}\text { Confirms the ability } \\
\text { of the novel } \\
\text { biomarker to detect } \\
\text { early stage disease }\end{array}$ & $\begin{array}{l}\text { Assess benefits } \\
\text { and harms }\end{array}$ \\
\hline 5 & $\begin{array}{l}\text { Cancer } \\
\text { control }\end{array}$ & $\begin{array}{l}\text { Randomized } \\
\text { study of new } \\
\text { biomarker } \\
\text { compared to US } \\
\text { and AFP }\end{array}$ & $\begin{array}{l}\text { Impact of screening } \\
\text { on reducing } \\
\text { mortality in patients } \\
\text { with cirrhosis (or } \\
\text { high-risk } \\
\text { populations) }\end{array}$ & $\begin{array}{lr}\text { Survival primary } \\
\text { endpoint; } \\
\text { secondary } \\
\text { early } \\
\text { detection, } r \text { assess } \\
\text { harms }\end{array}$ \\
\hline
\end{tabular}


Table 4. Guidelines recommendations for treatment according to levels of evidence* and strength of recommendation ${ }^{* *}$. Treatments accepted in guidelines (EASL(3) and $A A S L D(4)$ ) and level of evidence (modified from Llovet et al(119))

\begin{tabular}{|c|c|c|c|c|}
\hline Category & Treatment & $\begin{array}{l}\text { Eligibility criteria or } \\
\text { alternative approaches }\end{array}$ & $\begin{array}{l}\text { Evide } \\
\text { nce } \\
\text { level }\end{array}$ & $\begin{array}{l}\text { Recommendation } \\
\text { strength }\end{array}$ \\
\hline \multirow[t]{2}{*}{$\begin{array}{l}\text { Surgical } \\
\text { treatment }\end{array}$} & Resection & $\begin{array}{l}\text { Patient with solitary } \\
\text { tumors and well- } \\
\text { preserved liver function }\end{array}$ & $2 A$ & Strong \\
\hline & Liver transplantation & $\begin{array}{l}\text { Patients with single } \\
\text { tumors of } \leq 5 \mathrm{~cm} \text { or } \leq 3 \\
\text { nodules of } \geq 3 \mathrm{~cm} \text { (Milan } \\
\text { criteria) not suitable for } \\
\text { resection. Down staging } \\
\text { to Milan * }\end{array}$ & $2 \mathrm{~A}$ & $\begin{array}{l}\text { Strong; } \\
\text { Moderate: down } \\
\text { staging (US), not } \\
\text { recommended in } \\
\text { Europe }\end{array}$ \\
\hline \multirow[t]{2}{*}{$\begin{array}{l}\text { Loco- } \\
\text { regional } \\
\text { treatment }\end{array}$} & Local-ablation & $\begin{array}{l}\text { Radiofrequency, BCLC } \\
0-A \text { not suitable for } \\
\text { surgery, upper limit 4- } \\
5 \mathrm{~cm} \\
\text { Radiofrequency ablation } \\
\text { (and alternatively } \\
\text { percutaneous ethanol) } \\
\text { injection for patients with } \\
\text { BCLC 0-A tumours that } \\
\text { are not suitable for } \\
\text { surgery. }\end{array}$ & $\begin{array}{l}2 \mathrm{~A} \\
2 \mathrm{~B}\end{array}$ & Strong \\
\hline & Chemoembolization & $\begin{array}{l}\text { BCLC B (multinodular } \\
\text { asymptomatic tumours } \\
\text { without vascular invasion } \\
\text { or extra-hepatic spread) }\end{array}$ & $1 \mathrm{~A}$ & Strong \\
\hline \multirow[t]{2}{*}{$\begin{array}{l}\text { Systemic } \\
\text { treatment }\end{array}$} & $\begin{array}{l}\text { Atezolizumab+ } \\
\text { bevacizumab }\end{array}$ & $\begin{array}{l}\text { Child-Pugh A } \\
\text { Advanced HCC tumours } \\
\text { (BCLC C) or BCLC B } \\
\text { progressing upon loco- } \\
\text { regional therapies }\end{array}$ & $1 \mathrm{~A}$ & $\begin{array}{l}\text { Strong (not yet } \\
\text { included } \\
\text { guidelines) }\end{array}$ \\
\hline & Sorafenib & Child-Pugh A & $1 \mathrm{~A}$ & Strong \\
\hline
\end{tabular}




\begin{tabular}{|c|c|c|c|c|}
\hline & & $\begin{array}{l}\text { Advanced HCC tumours } \\
\text { (BCLC C) or BCLC B } \\
\text { progressing upon loco- } \\
\text { regional therapies }\end{array}$ & & \\
\hline & Lenvatinib & $\begin{array}{l}\text { Child-Pugh A } \\
\text { Advanced HCC tumours } \\
\text { (BCLC C) or BCLC B } \\
\text { progressing upon loco- } \\
\text { regional therapies. No } \\
\text { Main portal vein invasion }\end{array}$ & $1 \mathrm{~A}$ & Strong \\
\hline & Regorafenib & $\begin{array}{l}\text { Child-Pugh A } \\
\text { Tolerant to sorafenib. } \\
\text { Advanced HCC } \\
\text { progressing on sorafenib }\end{array}$ & $1 \mathrm{~A}$ & Strong \\
\hline & Ramucirumab & $\begin{array}{l}\text { Child-Pugh A } \\
\text { Advanced HCC } \\
\text { progressing on sorafenib } \\
\text { AFP> } 400 \mathrm{ng} / \mathrm{dL}\end{array}$ & $1 \mathrm{~A}$ & Strong \\
\hline & Cabozantinib & $\begin{array}{l}\text { Child-Pugh A } \\
\text { Advanced HCC } \\
\text { progressing on sorafenib }\end{array}$ & $1 \mathrm{~A}$ & Strong \\
\hline $\begin{array}{l}\text { Palliative } \\
\text { care }\end{array}$ & Palliative support & $\begin{array}{l}\text { Patients with BCLC D } \\
\text { tumours should receive } \\
\text { management of pain, } \\
\text { nutrition and } \\
\text { psychological support }\end{array}$ & $2 B$ & \\
\hline \multicolumn{5}{|c|}{$\begin{array}{l}\text { Treatments under investigation or with further evidence required to be adopted in } \\
\text { guidelines }\end{array}$} \\
\hline \multirow[t]{2}{*}{$\begin{array}{l}\text { Surgical } \\
\text { treatment }\end{array}$} & \multirow[t]{2}{*}{ Resection } & $\begin{array}{l}\text { Patients with multifocal } \\
\text { small tumors }(\leq 3 \\
\text { nodules } \leq 3 \mathrm{~cm}) \text { or mild } \\
\text { portal hypertension })\end{array}$ & $3 \mathrm{~A}$ & Moderate \\
\hline & & $\begin{array}{lr}\text { Adjuvant } & \text { treatments } \\
\text { after } & \text { resection/local } \\
\text { ablation } & \end{array}$ & $1 \mathrm{D}$ & $\begin{array}{l}\text { Strongly not } \\
\text { recommended }\end{array}$ \\
\hline
\end{tabular}




\begin{tabular}{|c|c|c|c|c|}
\hline & \multirow[t]{3}{*}{ Liver transplantation } & $\begin{array}{l}\text { Up-to-seven criteria in } \\
\text { patients without } \\
\text { microvascular invasion }\end{array}$ & \multirow{2}{*}{\begin{tabular}{|l|}
$2 \mathrm{~B}$ \\
$2 \mathrm{D}$
\end{tabular}} & \multirow{2}{*}{\begin{tabular}{|l|} 
Moderate \\
Moderate \\
\end{tabular}} \\
\hline & & $\begin{array}{l}\text { Neo-adjuvant loco- } \\
\text { regional therapies if the } \\
\text { waiting list exceeds } 6 \\
\text { months }\end{array}$ & & \\
\hline & & $\begin{array}{l}\text { Living donor liver } \\
\text { transplantation } \\
\text { patients with a waiting } \\
\text { list exceeding } 6-7 \\
\text { months }\end{array}$ & $2 A$ & Moderate \\
\hline \multirow[t]{5}{*}{$\begin{array}{l}\text { Loco- } \\
\text { regional } \\
\text { treatment }\end{array}$} & 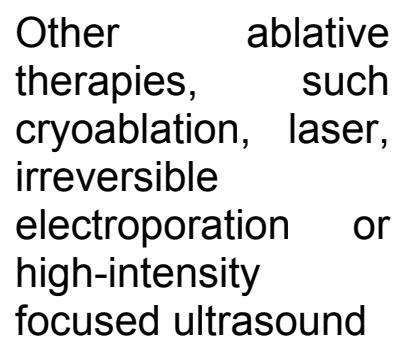 & $\begin{array}{l}\text { Patients with BCLC 0-A } \\
\text { tumours that are not } \\
\text { suitable for surgery }\end{array}$ & N/A & Not recommended \\
\hline & Chemoembolization & $\begin{array}{l}\text { Use of drug-eluting } \\
\text { beads, which has shown } \\
\text { similar response rates as } \\
\text { gelfoam-lipiodol particles } \\
\text { associated with less } \\
\text { systemic adverse events }\end{array}$ & $1 \mathrm{D}$ & Moderate \\
\hline & $\begin{array}{l}\text { Chemoembolization } \\
\text { combined } \quad \text { with } \\
\text { systemic TKis }\end{array}$ & $\begin{array}{l}\begin{array}{l}\text { Multiple RCT failed to } \\
\text { show }\end{array} \\
\text { outcomes }\end{array}$ & $1 \mathrm{~A}$ & Not recommended \\
\hline & $\begin{array}{l}\text { Y90- } \\
\text { Radioembolization- }\end{array}$ & $\begin{array}{l}\text { In patients at stage } \\
B C L C B \text { and in patients } \\
\text { BCLC A with a single } \\
\text { nodule larger than } 4 \mathrm{~cm} \\
\text { as an alternative to } \\
\text { resection }\end{array}$ & $1 \mathrm{D}$ & $\begin{array}{l}\text { No } \\
\text { recommendation }\end{array}$ \\
\hline & $\begin{array}{l}\text { External } \\
\text { conformal }\end{array}$ & $\begin{array}{l}\text { Single tumors at early } \\
\text { stages (BCLC A) }\end{array}$ & $3 A$ & $\begin{array}{l}\text { No } \\
\text { recommendation }\end{array}$ \\
\hline
\end{tabular}




\begin{tabular}{|c|c|c|c|c|}
\hline $\begin{array}{l}\text { Systemic } \\
\text { treatment }\end{array}$ & $\begin{array}{l}\text { Molecular targeted } \\
\text { therapies and } \\
\text { immune-based } \\
\text { therapies }\end{array}$ & $\begin{array}{l}\text { - Patients BCLC A as } \\
\text { neo-adjuvant therapies. } \\
\text { - Patients BCLC B in } \\
\text { combination with TACE, } \\
\text { Child Pugh A class, } \\
\text { ECOG } 0-1\end{array}$ & $1 \mathrm{~A}$ & $\begin{array}{l}\text { No } \\
\text { recommendation }\end{array}$ \\
\hline $\begin{array}{l}\text { Palliative } \\
\text { care }\end{array}$ & $\begin{array}{l}\text { Radiotherapy to } \\
\text { alleviate pain }\end{array}$ & $\begin{array}{l}\text { Patients with bone } \\
\text { metastasis }\end{array}$ & $3 \mathrm{~A}$ & Moderate \\
\hline \multicolumn{5}{|c|}{$\begin{array}{l}\text { AASLD, American Association for the Study of Liver Diseases; BCLC, Barcelona Clin } \\
\text { Liver Cancer Group; EASL, European Association for the Study of the Liver; HC } \\
\text { hepatocellular carcinoma. }\end{array}$} \\
\hline \multicolumn{5}{|c|}{$\begin{array}{l}{ }^{*} \text { National Cancer Institute classification: Strength of evidence: Level \#1 (RCT or meta- } \\
\text { analysis); \#2 non-randomized controlled studies; \#3 case series; Strength of end-point } \\
\text { A: survival B: cancer-specific survival; C Quality of life, and D: others. }{ }^{* *}\end{array}$} \\
\hline \multicolumn{5}{|c|}{ Table modified from EASL-EORTC guidelines(5) } \\
\hline
\end{tabular}




\begin{tabular}{|c|c|c|c|}
\hline \multicolumn{2}{|l|}{ Evaluation } & RECIST 1.1 & mRECIST \\
\hline \multirow{3}{*}{$\begin{array}{l}\text { Baseline } \\
\text { Assessment }\end{array}$} & $\begin{array}{l}\text { Target } \\
\text { lesions }\end{array}$ & $\begin{array}{l}\text { - Identify up to } 2 \text { intrahepatic } \\
\text { tumor lesions } \geq 1 \mathrm{~cm} \text {, that appear } \\
\text { suitable for accurate and repeat } \\
\text { assessments; measure their } \\
\text { longest viable tumor diameter. } \\
\text { - Identify extrahepatic tumor } \\
\text { lesions that are } \geq 1 \mathrm{~cm} \text { in longest } \\
\text { diameter and appear suitable for } \\
\text { accurate and repeat } \\
\text { assessments; measure their } \\
\text { longest overall tumor diameter. } \\
\text { - When selecting lymph nodes } \\
\text { as extrahepatic target lesions, } \\
\text { the short axis must be measured } \\
\text { and it must be } \geq 1.5 \mathrm{~cm} \text {. } \\
\text { - Overall, include a maximum of } \\
2 \text { target lesions per organ and } 5 \\
\text { target lesions in total. }\end{array}$ & $\begin{array}{l}\text { - Identify up to } 2 \text { intrahepatic tumor } \\
\text { lesions } \geq 1 \mathrm{~cm} \text {, that show typical } \\
\text { intratumoral arterial enhancement } \\
\text { and appear suitable for accurate } \\
\text { and repeat assessments; measure } \\
\text { their longest viable tumor diameter. } \\
\text { - Identify extrahepatic tumor lesions } \\
\text { (and intrahepatic lesions with } \\
\text { atypical enhancement in patients } \\
\text { without typical intrahepatic lesions) } \\
\text { that are } \geq 1 \mathrm{~cm} \text { in longest diameter } \\
\text { and appear suitable for accurate } \\
\text { and repeat assessments; measure } \\
\text { their longest overall tumor diameter. } \\
\text { - When selecting lymph nodes as } \\
\text { extrahepatic target lesions, the } \\
\text { short axis must be measured: it } \\
\text { must be } \geq 1.5 \mathrm{~cm} \text { for all lymph nodes } \\
\text { except for porta hepatis lymph } \\
\text { nodes where it is required that it is } \geq \\
2 \mathrm{~cm} \text {. }\end{array}$ \\
\hline & $\begin{array}{l}\text { Non- } \\
\text { target } \\
\text { lesions }\end{array}$ & $\begin{array}{l}\text { - Tumor lesions or sites of } \\
\text { disease that have not been } \\
\text { selected as target lesions should } \\
\text { be recorded at baseline as non- } \\
\text { target lesions. }\end{array}$ & $\begin{array}{l}\text { - Tumor lesions or sites of disease } \\
\text { that have not been selected as } \\
\text { target lesions should be recorded at } \\
\text { baseline as non-target lesions. } \\
\text { - Malignant portal vein thrombosis } \\
\text { should be considered as a non- } \\
\text { target lesions. } \\
\text { - Ascites and pleural effusions } \\
\text { should not be considered as tumor } \\
\text { lesions, unless associated with } \\
\text { unequivocal neoplastic peritoneal or } \\
\text { pleural nodules. }\end{array}$ \\
\hline & & $\begin{array}{l}\text { - Measure the longest overall } \\
\text { tumor diameter for intrahepatic }\end{array}$ & $\begin{array}{l}\text { - Measure the longest viable tumor } \\
\text { diameter of typical intrahepatic }\end{array}$ \\
\hline
\end{tabular}




\begin{tabular}{|c|c|c|c|}
\hline \multirow[t]{3}{*}{$\begin{array}{l}\text { Post- } \\
\text { Baseline } \\
\text { Assessments }\end{array}$} & $\begin{array}{l}\text { Target } \\
\text { lesions }\end{array}$ & $\begin{array}{l}\text { and non-nodal extrahepatic } \\
\text { target lesions, and the short axis } \\
\text { diameter for nodal target lesions. }\end{array}$ & $\begin{array}{l}\text { target lesions avoiding the inclusion } \\
\text { of any major intervening areas of } \\
\text { necrosis. } \\
\text { - Pay attention in distinguishing } \\
\text { areas of tumor necrosis from areas } \\
\text { of reduced arterial perfusion caused } \\
\text { by changes in local hemodynamics. } \\
\text { A change from hypervascularity to } \\
\text { hypovascularity does not represent } \\
\text { tumor necrosis. Only tumors or } \\
\text { tumor areas that show complete } \\
\text { absence of contrast enhancement } \\
\text { can be assumed to represent } \\
\text { necrotic tissue. } \\
\text { - Measure the longest overall tumor } \\
\text { diameter for atypical intrahepatic } \\
\text { target lesions and non-nodal } \\
\text { extrahepatic target lesions, and the } \\
\text { short axis diameter for nodal target } \\
\text { lesions }\end{array}$ \\
\hline & $\begin{array}{l}\text { Non- } \\
\text { target } \\
\text { lesions }\end{array}$ & $\begin{array}{l}\text { - Qualitative assessment of } \\
\text { response. }\end{array}$ & $\begin{array}{l}\text { - Qualitative assessment of } \\
\text { response, taking into account tumor } \\
\text { necrosis for typical intrahepatic non- } \\
\text { target lesions. } \\
\text { - Complete disappearance of } \\
\text { enhancement inside malignant } \\
\text { portal vein thrombus should be } \\
\text { considered equivalent to complete } \\
\text { regression }\end{array}$ \\
\hline & $\begin{array}{l}\text { New } \\
\text { lesions }\end{array}$ & $\begin{array}{l}\text { Any new lesion that has no } \\
\text { corresponding lesion on baseline } \\
\text { imaging and is unequivocally } \\
\text { malignant is considered as } \\
\text { evidence of PD. }\end{array}$ & $\begin{array}{l}\text { - By definition, a new lesion has no } \\
\text { corresponding lesion on the } \\
\text { baseline imaging. } \\
\text { - A new liver lesion } \geq 1 \mathrm{~cm} \text { that } \\
\text { shows nonrim-like } \\
\text { hypervascularization in the arterial } \\
\text { phase with nonperipheral washout } \\
\text { in the portal venous or the delayed } \\
\text { phase meets the criteria for } \\
\text { unequivocal new lesion and } \\
\text { declares PD. } \\
\text { - Any new liver lesion }<1 \mathrm{~cm} \text { or any } \\
\text { new liver lesion of any size that fails } \\
\text { to show the enhancement pattern } \\
\text { described above should be } \\
\text { considered as equivocal and can } \\
\text { only be diagnosed as HCC by } \\
\text { evidence of either a change in } \\
\text { enhancement pattern (when } \geq 1 \mathrm{~cm} \text { ) } \\
\text { or an interval growth } \geq 1 \mathrm{~cm} \text { in } \\
\text { subsequent scans. } \\
\text { - If an equivocal new lesion is later } \\
\text { determined to be unequivocal, the } \\
\text { timepoint of progression will be the }\end{array}$ \\
\hline
\end{tabular}




\begin{tabular}{|l|l|l|l|}
\hline & & $\mid \begin{array}{l}\text { timepoint that the lesion was first } \\
\text { noted as equivocal. } \\
\text { - Ascites or pleural effusion that } \\
\text { appear during treatment should not } \\
\text { be assumed to represent PD, } \\
\text { unless associated with the } \\
\text { emergence of unequivocal } \\
\text { neoplastic peritoneal or pleural } \\
\text { nodules. }\end{array}$ \\
\hline
\end{tabular}

Overall assessment of tumor response by RECIST 1.1 or $\mathrm{mRECIST}$

Target lesions Non-target lesions New lesions Overall response

$\begin{array}{llll}\text { CR } & \text { CR } & \text { No } & \text { CR } \\ \text { CR } & \text { Non-CR-NonPD } & \text { No } & \text { PR } \\ \text { PR } & \text { Non-PD } & \text { No } & \text { PR } \\ \text { SD } & \text { Non-PD } & \text { No } & \text { SD } \\ \text { PD } & \text { Any } & \text { Yes or no } & \text { PD } \\ \text { Any } & \text { PD } & \text { Yes or no } & \text { PD } \\ \text { Any } & \text { Any } & \text { Yes } & \text { PD }\end{array}$

CR, complete response; PR, partial response; SD, stable disease; PD, progressive disease. 


\section{Table 6. Unmet needs in trial design in HCC.}

1. Clinical trials:

- Evidence-based data from RCT to provide standard of care in

a) Adjuvant setting after resection/local ablation

b) Neo-adjuvant setting prior resection/liver transplantation

c) Define role of loco-regional therapies or SBRT in large single nonsurgical tumors

- Evidence-based data from RCT to improve the standard of care in

a) Combination or systemic therapies to improve chemoembolization in intermediate HCC

b) Combination (or triple) therapies for $1^{\text {st }}$ line advanced HCC

c) Molecular and immune-based therapies for patients with HCC and impaired liver function (Child-Pugh B).

- Pivotal proof-of-concept phase II trials and trial enrichment for oncogenic drivers or signaling pathways

- Systematic inclusion of cost-benefit analyses

2. Identification and validation of biomarkers:

- Develop biomarkers for early detection in surveillance programs

- Identify biomarkers predicting treatment response and primary resistance (tissue or liquid biopsy)

3. Quality of life \& patient reported outcomes:

- Incorporate tools for measuring quality of life into clinical trial design as an endpoint

- Systematic inclusion of patient reported outcomes

4. Molecular pathogenesis and drug development:

- Integrate molecular subclasses to the clinical staging system in order to better guide treatment allocation

- Target oncogene addiction loops that result from DNA amplifications and gene mutations or overexpression

- Improve models for pre-clinical testing of novel drugs 


\section{Table 7. Variables to be included in clinical trials assessing treatments for HCC patients}

Demographic

Tumor description

Staging system

Liver function

General health

\begin{abstract}
Age, sex, ethnicity
Underlying liver disease (cirrhosis, chronic hepatitis)

Etiology : define based upon $\mathrm{HCV}, \mathrm{HBV}$, alcohol, NASH-NAFLD and others.
\end{abstract}

Radiological characteristics: size, number of nodules, macroscopic vascular invasion, extrahepatic spread

Alpha-fetoprotein

Pathological characteristics in adjuvant trials: size, number, differentiation degree, satellites, micro and macroscopic vascular invasion, pTNM

BCLC staging classification

Bilirubin, aminotransferases, albumin, alkaline phosphatase, gamma-glutamyl transpeptidase, serum creatinine, serum sodium, prothrombin time, INR, platelet count

Presence of ascites or encephalopathy

Child - Pugh score

ALBI and MELD score

ECOG status, pain, constitutional syndrome

\footnotetext{
* Modified from Llovet et al, JNCI 2008(12) HCV = hepatitis C virus; HBV = hepatitis B virus; pTNM = pathological tumor-node-metastasis;

BCLC = Barcelona Clinic Liver Cancer; BUN = serum urea nitrogen; MELD: Model of EndStage Liver Disease; ECOG = Eastern Cooperative

Oncology Group.
} 


\section{REFERENCES}

1. Bray F, Ferlay J, Soerjomataram I, Siegel RL, Torre LA, Jemal A. Global cancer statistics 2018: GLOBOCAN estimates of incidence and mortality worldwide for 36 cancers in 185 countries. CA Cancer J. Clin. 2018; Available from: http://dx.doi.org/10.3322/caac.21492

2. Villanueva A. Hepatocellular Carcinoma. N. Engl. J. Med. 2019;380:1450-1462.

3. Galle PR, Forner A, Llovet JM, Mazzaferro V, Piscaglia F, Raoul J-L, et al. EASL Clinical Practice Guidelines: Management of hepatocellular carcinoma. J. Hepatol. 2018, 56(4):908-43

4. Marrero JA, Kulik LM, Sirlin CB, Zhu AX, Finn RS, Abecassis MM, et al. Diagnosis, Staging, and Management of Hepatocellular Carcinoma: 2018 Practice Guidance by the American Association for the Study of Liver Diseases. Hepatology. 2018;68:723750 .

5. Llovet JM, Ducreux M, Lencioni R, Di Bisceglie AM, Galle PR, Dufour JF, et al. EASLEORTC clinical practice guidelines: management of hepatocellular carcinoma. J. Hepatol. 2012;56:908-943.

6. Llovet JM, Montal R, Sia D, Finn RS. Molecular therapies and precision medicine for hepatocellular carcinoma. Nat. Rev. Clin. Oncol. [Internet]. 2018;Available from: http://dx.doi.org/10.1038/s41571-018-0073-4

7. Kudo M, Finn RS, Qin S, Han K-H, Ikeda K, Piscaglia F, et al. Lenvatinib versus sorafenib in first-line treatment of patients with unresectable hepatocellular carcinoma: a randomised phase 3 non-inferiority trial. Lancet. 2018;391:1163-1173.

8. Bruix J, Qin S, Merle P, Granito A, Huang Y-H, Bodoky G, et al. Regorafenib for patients with hepatocellular carcinoma who progressed on sorafenib treatment (RESORCE): a randomised, double-blind, placebo-controlled, phase 3 trial. Lancet. 2017;389:56-66.

9. Abou-Alfa GK, Meyer T, Cheng A-L, El-Khoueiry AB, Rimassa L, Ryoo B-Y, et al. Cabozantinib in Patients with Advanced and Progressing Hepatocellular Carcinoma. N. Engl. J. Med. 2018;379:54-63.

10. Zhu AX, Kang Y-K, Yen C-J, Finn RS, Galle PR, Llovet JM, et al. Ramucirumab after sorafenib in patients with advanced hepatocellular carcinoma and increased $\alpha-$ fetoprotein concentrations (REACH-2): a randomised, double-blind, placebocontrolled, phase 3 trial. Lancet Oncol. [Internet]. 2019;Available from: http://dx.doi.org/10.1016/S1470-2045(18)30937-9

11. Llovet JM, Ricci S, Mazzaferro V, Hilgard P, Gane E, Blanc J-F, et al. Sorafenib in advanced hepatocellular carcinoma. N. Engl. J. Med. 2008;359:378-390.

12. Llovet JM, Di Bisceglie AM, Bruix J, Kramer BS, Lencioni R, Zhu AX, et al. Design and endpoints of clinical trials in hepatocellular carcinoma. JNCl Journal of the National Cancer Institute. 2008;100:698-711.

13. Lee $\mathrm{DH}$, Vielemeyer $\mathrm{O}$. Analysis of overall level of evidence behind Infectious Diseases Society of America practice guidelines. Arch. Intern. Med. 2011;171:18-22. 
14. Jadad AR, Moore RA, Carroll D, Jenkinson C, Reynolds DJ, Gavaghan DJ, et al. Assessing the quality of reports of randomized clinical trials: is blinding necessary? Control. Clin. Trials. 1996;17:1-12.

15. Verhagen AP, de Vet HC, de Bie RA, Kessels AG, Boers M, Bouter LM, et al. The Delphi list: a criteria list for quality assessment of randomized clinical trials for conducting systematic reviews developed by Delphi consensus. J. Clin. Epidemiol. 1998;51:1235-1241.

16. Altman DG, Schulz KF, Moher D, Egger M, Davidoff F, Elbourne D, et al. The revised CONSORT statement for reporting randomized trials: explanation and elaboration. Ann. Intern. Med. 2001;134:663-694.

17. Piaggio G, Elbourne DR, Altman DG, Pocock SJ, Evans SJW, CONSORT Group. Reporting of noninferiority and equivalence randomized trials: an extension of the CONSORT statement. JAMA. 2006;295:1152-1160.

18. van Tulder M, Furlan A, Bombardier C, Bouter L, Editorial Board of the Cochrane Collaboration Back Review Group. Updated method guidelines for systematic reviews in the cochrane collaboration back review group. Spine. 2003;28:1290-1299.

19. Lopez PM, Villanueva A, Llovet JM. Systematic review: evidence-based management of hepatocellular carcinoma - an updated analysis of randomized controlled trials. Aliment. Pharmacol. Ther. 2006;23:1535-1547.

20. Llovet JM, Montal R, Villanueva A. Randomized trials and endpoints in advanced HCC: Role of PFS as a surrogate of survival. J. Hepatol. [Internet]. 2019;Available from: http://dx.doi.org/10.1016/j.jhep.2019.01.028

21. Lencioni R, Llovet JM. Modified RECIST (mRECIST) assessment for hepatocellular carcinoma. Semin. Liver Dis. 2010;30:52-60.

22. Cheng AL, Kang YK, Lin DY, Park JW, Kudo M, Qin S, et al. Sunitinib versus sorafenib in advanced hepatocellular cancer: results of a randomized phase III trial. J. Clin. Oncol. 2013;31:4067-4075.

23. Finn RS, Merle P, Granito A, Huang Y-H, Bodoky G, Pracht M, et al. Outcomes of sequential treatment with sorafenib followed by regorafenib for HCC: additional analyses from the phase 3 RESORCE trial. J. Hepatol. [Internet]. 2018;Available from: http://dx.doi.org/10.1016/j.jhep.2018.04.010

24. Checkmate 459 results [Internet]. Available from: https://news.bms.com/pressrelease/bmy/bristol-myers-squibb-announces-results-checkmate-459-studyevaluating-opdivo-nivol

25. Prasad V, Kim C, Burotto M, Vandross A. The Strength of Association Between Surrogate End Points and Survival in Oncology: A Systematic Review of Trial-Level Meta-analyses. JAMA Intern. Med. 2015;175:1389-1398.

26. Eisenhauer EA, Therasse P, Bogaerts J, Schwartz LH, Sargent D, Ford R, et al. New response evaluation criteria in solid tumours: revised RECIST guideline (version 1.1). European journal of cancer (Oxford, England : 1990). 2009;45:228-247.

27. Llovet JM, Lencioni R. mRECIST for HCC: Performance and novel refinements. J. 
Hepatol. 2020;72:288-306.

28. Edeline J, Boucher E, Rolland Y, Vauléon E, Pracht M, Perrin C, et al. Comparison of tumor response by Response Evaluation Criteria in Solid Tumors (RECIST) and modified RECIST in patients treated with sorafenib for hepatocellular carcinoma. Cancer. 2012;118:147-156.

29. Ronot M, Bouattour M, Wassermann J, Bruno O, Dreyer C, Larroque B, et al. Alternative Response Criteria (Choi, European Association for the Study of the Liver, and Modified Response Evaluation Criteria in Solid Tumors [RECIST]) Versus RECIST 1.1 in Patients With Advanced Hepatocellular Carcinoma Treated With Sorafenib. Oncologist. 2014;19:394-402.

30. Takada J, Hidaka H, Nakazawa T, Kondo M, Numata K, Tanaka K, et al. Modified response evaluation criteria in solid tumors is superior to response evaluation criteria in solid tumors for assessment of responses to sorafenib in patients with advanced hepatocellular carcinoma. BMC Res. Notes. 2015;8:609.

31. Lencioni R, Montal R, Torres F, Park J-W, Decaens T, Raoul J-L, et al. Objective response by $\mathrm{mRECIST}$ as a predictor and potential surrogate end-point of overall survival in advanced HCC. J. Hepatol. 2017;66:1166-1172.

32. Meyer T, Palmer DH, Cheng A-L, Hocke J, Loembé A-B, Yen C-J. mRECIST to predict survival in advanced hepatocellular carcinoma: Analysis of two randomised phase II trials comparing nintedanib vs sorafenib. Liver Int. 2017;37:1047-1055.

33. Burzykowski T, Buyse M. Surrogate threshold effect: an alternative measure for metaanalytic surrogate endpoint validation. Pharm. Stat. 2006;5:173-186.

34. Reig M, Rimola J, Torres F, Darnell A, Rodriguez-Lope C, Forner A, et al. Postprogression survival of patients with advanced hepatocellular carcinoma: Rationale for second-line trial design. HEPATOLOGY, Vol. 00, No. X, 2013 REIG ET AL. 2013;58:2023-2031.

35. Wolchok JD, Hoos A, O'Day S, Weber JS, Hamid O, Lebbé C, et al. Guidelines for the evaluation of immune therapy activity in solid tumors: immune-related response criteria. Clin. Cancer Res. 2009;15:7412-7420.

36. Seymour L, Bogaerts J, Perrone A, Ford R, Schwartz LH, Mandrekar S, et al. iRECIST: guidelines for response criteria for use in trials testing immunotherapeutics. Lancet Oncol. 2017;18:e143-e152.

37. Gyawali B, Kesselheim AS. Reinforcing the social compromise of accelerated approval. Nat. Rev. Clin. Oncol. [Internet]. 2018;Available from: http://dx.doi.org/10.1038/s41571-018-0066-3

38. El-Khoueiry AB, Sangro B, Yau T, Crocenzi TS, Kudo M, Hsu C, et al. Nivolumab in patients with advanced hepatocellular carcinoma (CheckMate 040): an open-label, non-comparative, phase $1 / 2$ dose escalation and expansion trial. Lancet [Internet]. 2017;Available from: http://dx.doi.org/10.1016/S0140-6736(17)31046-2

39. Zhu AX, Finn RS, Edeline J, Cattan S, Ogasawara S, Palmer D, et al. Pembrolizumab in patients with advanced hepatocellular carcinoma previously treated with sorafenib (KEYNOTE-224): a non-randomised, open-label phase 2 trial. Lancet Oncol. 
[Internet]. 2018;Available from: http://dx.doi.org/10.1016/S1470-2045(18)30351-6

40. Center for Drug Evaluation, Research. FDA grants accelerated approval to nivolumab and ipilimumab combinatio [Internet]. U.S. Food and Drug Administration. 2020 [cited 2020 Mar 26];Available from: http://www.fda.gov/drugs/resources-informationapproved-drugs/fda-grants-accelerated-approval-nivolumab-and-ipilimumabcombination-hepatocellular-carcinoma

41. Finn RS, Ryoo B-Y, Merle P, Kudo M, Bouattour M, Lim HY, et al. Pembrolizumab As Second-Line Therapy in Patients With Advanced Hepatocellular Carcinoma in KEYNOTE-240: A Randomized, Double-Blind, Phase III Trial. J. Clin. Oncol. 2019;JCO1901307.

42. Ellis LM, Bernstein DS, Voest EE, Berlin JD, Sargent D, Cortazar P, et al. American Society of Clinical Oncology perspective: Raising the bar for clinical trials by defining clinically meaningful outcomes. J. Clin. Oncol. 2014;32:1277-1280.

43. Nault J-C, Cheng A-L, Sangro B, Llovet JM. Milestones in the pathogenesis and management of primary liver cancer. J. Hepatol. 2020;72:209-214.

44. Singal $A G$, Lampertico $P$, Nahon $P$. Epidemiology and surveillance for hepatocellular carcinoma: New trends. J. Hepatol. 2020;72:250-261.

45. Tzartzeva K, Obi J, Rich NE, Parikh ND, Marrero JA, Yopp A, et al. Surveillance Imaging and Alpha Fetoprotein for Early Detection of Hepatocellular Carcinoma in Patients With Cirrhosis: A Meta-analysis. Gastroenterology. 2018;154:1706-1718.e1.

46. Atiq O, Tiro J, Yopp AC, Muffler A, Marrero JA, Parikh ND, et al. An assessment of benefits and harms of hepatocellular carcinoma surveillance in patients with cirrhosis. Hepatology. 2017;65:1196-1205.

47. Park J-W, Chen M, Colombo M, Roberts LR, Schwartz M, Chen P-J, et al. Global patterns of hepatocellular carcinoma management from diagnosis to death: the BRIDGE Study. Liver Int. 2015;35:2155-2166.

48. Totoki $\mathrm{Y}$, Tatsuno K, Covington KR, Ueda H, Creighton CJ, Kato M, et al. Transancestry mutational landscape of hepatocellular carcinoma genomes. Nat. Genet. 2014;46:1267-1273.

49. Schulze K, Imbeaud S, Letouzé E, Alexandrov LB, Calderaro J, Rebouissou S, et al. Exome sequencing of hepatocellular carcinomas identifies new mutational signatures and potential therapeutic targets. Nat. Genet. 2015;47:505-511.

50. Ally A, Balasundaram M, Carlsen R, Chuah E, Clarke A, Dhalla N, et al. Comprehensive and Integrative Genomic Characterization of Hepatocellular Carcinoma. Cell. 2017;169:1327-1341.e23.

51. Gao Q, Zhu H, Dong L, Shi W, Chen R, Song Z, et al. Integrated Proteogenomic Characterization of HBV-Related Hepatocellular Carcinoma. Cell. 2019;179:561577.e22.

52. Johnson PJ, Pirrie SJ, Cox TF, Berhane S, Teng M, Palmer D, et al. The detection of hepatocellular carcinoma using a prospectively developed and validated model based on serological biomarkers. Cancer Epidemiol. Biomarkers Prev. 2014;23:144-153. 
53. Wang $M$, Sanda $M$, Comunale MA, Herrera $H$, Swindell $C$, Kono $Y$, et al. Changes in the Glycosylation of Kininogen and the Development of a Kininogen-Based Algorithm for the Early Detection of HCC. Cancer Epidemiol. Biomarkers Prev. 2017;26:795803.

54. von Felden J, Craig AJ, Villanueva A. Role of circulating tumor DNA to help decisionmaking in hepatocellular carcinoma. Oncoscience. 2018;5:209-211.

55. Kisiel JB, Dukek BA, Kanipakam RVSR, Ghoz HM, Yab TC, Berger CK, et al. Hepatocellular Carcinoma Detection by Plasma Methylated DNA: Discovery, Phase I Pilot, and Phase II Clinical Validation. Hepatology [Internet]. 2018;Available from: http://dx.doi.org/10.1002/hep.30244

56. Khatri G, Pedrosa I, Ananthakrishnan L, de Leon AD, Fetzer DT, Leyendecker J, et al. Abbreviated-protocol screening MRI vs. complete-protocol diagnostic MRI for detection of hepatocellular carcinoma in patients with cirrhosis: An equivalence study using LI-RADS v2018. J. Magn. Reson. Imaging [Internet]. 2019;Available from: http://dx.doi.org/10.1002/jmri.26835

57. Pepe MS, Etzioni R, Feng Z, Potter JD, Thompson ML, Thornquist M, et al. Phases of biomarker development for early detection of cancer. J. Natl. Cancer Inst. 2001;93:1054-1061.

58. Pepe MS, Feng Z, Janes H, Bossuyt PM, Potter JD. Pivotal evaluation of the accuracy of a biomarker used for classification or prediction: standards for study design. J. Natl. Cancer Inst. 2008;100:1432-1438.

59. Ioannou GN, Green P, Lowy E, Mun EJ, Berry K. Differences in hepatocellular carcinoma risk, predictors and trends over time according to etiology of cirrhosis. PLoS One. 2018;13:e0204412.

60. Kanwal F, Kramer JR, Mapakshi S, Natarajan Y, Chayanupatkul M, Richardson PA, et al. Risk of Hepatocellular Cancer in Patients With Non-Alcoholic Fatty Liver Disease. Gastroenterology. 2018;155:1828-1837.e2.

61. Kanwal F, Singal AG. Surveillance for Hepatocellular Carcinoma: Current Best Practice and Future Direction. Gastroenterology [Internet]. 2019;Available from: http://dx.doi.org/10.1053/j.gastro.2019.02.049

62. Yang H-I, Yeh M-L, Wong GL, Peng C-Y, Chen C-H, Trinh HN, et al. REAL-B (Realworld Effectiveness from the Asia Pacific Rim Liver Consortium for HBV) Risk Score for the Prediction of Hepatocellular Carcinoma in Chronic Hepatitis B Patients Treated with Oral Antiviral Therapy. J. Infect. Dis. [Internet]. 2019;Available from: http://dx.doi.org/10.1093/infdis/jiz477

63. Office of the Commissioner. Biomarkers at FDA [Internet]. U.S. Food and Drug Administration. 2019 [cited 2019 Nov 15];Available from: http://www.fda.gov/scienceresearch/about-science-research-fda/biomarkers-fda

64. Goodsaid FM. The Labyrinth of Product Development and Regulatory Approvals in Liquid Biopsy Diagnostics. Clin. Transl. Sci. 2019;12:431-439.

65. Simon TG, Ma Y, Ludvigsson JF, Chong DQ, Giovannucci EL, Fuchs CS, et al. Association Between Aspirin Use and Risk of Hepatocellular Carcinoma. JAMA 
Oncol. 2018;4:1683-1690.

66. Malehmir M, Pfister D, Gallage S, Szydlowska M, Inverso D, Kotsiliti E, et al. Platelet GPIba is a mediator and potential interventional target for NASH and subsequent liver cancer. Nat. Med. [Internet]. 2019;Available from: http://dx.doi.org/10.1038/s41591019-0379-5

67. Simon TG, Duberg A-S, Aleman S, Hagstrom H, Nguyen LH, Khalili H, et al. Lipophilic Statins and Risk for Hepatocellular Carcinoma and Death in Patients With Chronic Viral Hepatitis: Results From a Nationwide Swedish Population. Ann. Intern. Med. 2019;171:318-327.

68. Tseng $\mathrm{C}-\mathrm{H}$. Metformin and risk of hepatocellular carcinoma in patients with type 2 diabetes. Liver Int. 2018;38:2018-2027.

69. Heimbach JK, Kulik LM, Finn RS, Sirlin CB, Abecassis MM, Roberts LR, et al. AASLD guidelines for the treatment of hepatocellular carcinoma. Hepatology. 2018;67:358380.

70. Roayaie S, Jibara G, Tabrizian P, Park J-W, Yang J, Yan L, et al. The role of hepatic resection in the treatment of hepatocellular cancer. Hepatology. 2015;62:440-451.

71. Roayaie S, Obeidat K, Sposito C, Mariani L, Bhoori S, Pellegrinelli A, et al. Resection of hepatocellular cancer $\leq 2 \mathrm{~cm}$ : results from two Western centers. Hepatology. 2013;57:1426-1435.

72. Muto Y, Moriwaki H, Ninomiya M, Adachi S, Saito A, Takasaki KT, et al. Prevention of second primary tumors by an acyclic retinoid, polyprenoic acid, in patients with hepatocellular carcinoma. Hepatoma Prevention Study Group. N. Engl. J. Med. 1996;334:1561-1567.

73. Okita K, Izumi N, Matsui O, Tanaka K, Kaneko S, Moriwaki H, et al. Peretinoin after curative therapy of hepatitis C-related hepatocellular carcinoma: a randomized double-blind placebo-controlled study. J. Gastroenterol. 2015;50:191-202.

74. Takayama T, Sekine T, Makuuchi M, Yamasaki S, Kosuge T, Yamamoto J, et al. Adoptive immunotherapy to lower postsurgical recurrence rates of hepatocellular carcinoma: a randomised trial. Lancet. 2000;356:802-807.

75. Lau WY, Lai ECH, Leung TWT, Yu SCH. Adjuvant intra-arterial iodine-131-labeled lipiodol for resectable hepatocellular carcinoma: a prospective randomized trialupdate on 5-year and 10-year survival. Ann. Surg. 2008;247:43-48.

76. Bruix J, Takayama T, Mazzaferro V, Chau GY, Yang J, Kudo M, et al. Adjuvant sorafenib for hepatocellular carcinoma after resection or ablation (STORM): a phase 3, randomised, double-blind, placebo-controlled trial. Lancet Oncol. 2015;16:13441354.

77. Bott MJ, Yang SC, Park BJ, Adusumilli PS, Rusch VW, Isbell JM, et al. Initial results of pulmonary resection after neoadjuvant nivolumab in patients with resectable nonsmall cell lung cancer. J. Thorac. Cardiovasc. Surg. 2019;158:269-276.

78. Mazzaferro V, Regalia E, Doci R, Andreola S, Pulvirenti A, Bozzetti F, et al. Liver transplantation for the treatment of small hepatocellular carcinomas in patients with 
cirrhosis. N. Engl. J. Med. 1996;334:693-699.

79. Yao FY, Mehta N, Flemming J, Dodge J, Hameed B, Fix O, et al. Downstaging of hepatocellular cancer before liver transplant: long-term outcome compared to tumors within Milan criteria. Hepatology. 2015;61:1968-1977.

80. Sposito C, Mariani L, Germini A, Flores Reyes M, Bongini M, Grossi G, et al. Comparative efficacy of sorafenib versus best supportive care in recurrent hepatocellular carcinoma after liver transplantation: a case-control study. J. Hepatol. 2013;59:59-66.

81. Morales RE, Shoushtari AN, Walsh MM, Grewal P, Lipson EJ, Carvajal RD. Safety and efficacy of ipilimumab to treat advanced melanoma in the setting of liver transplantation. J Immunother Cancer. 2015;3:22.

82. Breen DJ, Lencioni R. Image-guided ablation of primary liver and renal tumours. Nat. Rev. Clin. Oncol. 2015;12:175-186.

83. Cho YK, Kim JK, Kim MY, Rhim H, Han JK. Systematic review of randomized trials for hepatocellular carcinoma treated with percutaneous ablation therapies. Hepatology. 2009;49:453-459.

84. Glassberg MB, Ghosh S, Clymer JW, Qadeer RA, Ferko NC, Sadeghirad B, et al. Microwave ablation compared with radiofrequency ablation for treatment of hepatocellular carcinoma and liver metastases: a systematic review and metaanalysis. Onco. Targets. Ther. 2019;12:6407-6438.

85. Nault J-C, Sutter O, Nahon P, Ganne-Carrié N, Séror O. Percutaneous treatment of hepatocellular carcinoma: State of the art and innovations. J. Hepatol. 2018;68:783797.

86. Llovet JM, Real MI, Montaña X, Planas R, Coll S, Aponte J, et al. Arterial embolisation or chemoembolisation versus symptomatic treatment in patients with unresectable hepatocellular carcinoma: a randomised controlled trial. Lancet. 2002;359:1734-1739.

87. Lo C-M, Ngan H, Tso W-K, Liu C-L, Lam C-M, Poon RT-P, et al. Randomized controlled trial of transarterial lipiodol chemoembolization for unresectable hepatocellular carcinoma. Hepatology. 2002;35:1164-1171.

88. Llovet JM, Bruix J. Systematic review of randomized trials for unresectable hepatocellular carcinoma: Chemoembolization improves survival. Hepatology. 2003;37:429-442.

89. Salem R, Mazzaferro V, Sangro B. Yttrium 90 radioembolization for the treatment of hepatocellular carcinoma: biological lessons, current challenges, and clinical perspectives. Hepatology. 2013;58:2188-2197.

90. Bolondi L, Burroughs A, Dufour J-F, Galle PR, Mazzaferro V, Piscaglia F, et al. Heterogeneity of patients with intermediate (BCLC B) Hepatocellular Carcinoma: proposal for a subclassification to facilitate treatment decisions. Semin. Liver Dis. 2012;32:348-359.

91. Kudo M, Arizumi T, Ueshima K, Sakurai T, Kitano M, Nishida N. Subclassification of 
BCLC B Stage Hepatocellular Carcinoma and Treatment Strategies: Proposal of Modified Bolondi's Subclassification (Kinki Criteria). Dig. Dis. 2015;33:751-758.

92. Forner A, Gilabert M, Bruix J, Raoul JL. Treatment of intermediate-stage hepatocellular carcinoma. Nat. Rev. Clin. Oncol. 2014;11:525-535.

93. Meyer T, Fox R, Ma YT, Ross PJ, James MW, Sturgess R, et al. Sorafenib in combination with transarterial chemoembolisation in patients with unresectable hepatocellular carcinoma (TACE 2): a randomised placebo-controlled, double-blind, phase 3 trial. Lancet Gastroenterol Hepatol. 2017;2:565-575.

94. Lencioni R, Llovet JM, Han G, Tak WY, Yang J, Guglielmi A, et al. Sorafenib or placebo plus TACE with doxorubicin-eluting beads for intermediate stage HCC: The SPACE trial. J. Hepatol. 2016;64:1090-1098.

95. Kudo M, Han G, Finn RS, Poon RT, Blanc JF, Yan L, et al. Brivanib as adjuvant therapy to transarterial chemoembolization in patients with hepatocellular carcinoma: A randomized phase III trial. Hepatology. 2014;60:1697-1707.

96. Kudo M, Cheng A-L, Park J-W, Park JH, Liang P-C, Hidaka H, et al. Orantinib versus placebo combined with transcatheter arterial chemoembolisation in patients with unresectable hepatocellular carcinoma (ORIENTAL): a randomised, double-blind, placebo-controlled, multicentre, phase 3 study. Lancet Gastroenterol Hepatol. 2018;3:37-46.

97. Johnson PJ, Berhane S, Kagebayashi C, Satomura S, Teng M, Reeves HL, et al. Assessment of liver function in patients with hepatocellular carcinoma: a new evidence-based approach-the ALBI grade. J. Clin. Oncol. 2015;33:550-558.

98. Edeline J, Blanc J-F, Johnson P, Campillo-Gimenez B, Ross P, Ma YT, et al. A multicentre comparison between Child Pugh and Albumin-Bilirubin scores in patients treated with sorafenib for Hepatocellular Carcinoma. Liver Int. 2016;36:1821-1828.

99. Kadalayil L, Benini R, Pallan L, O’Beirne J, Marelli L, Yu D, et al. A simple prognostic scoring system for patients receiving transarterial embolisation for hepatocellular cancer. Ann. Oncol. 2013;24:2565-2570.

100. Waked I, Berhane S, Toyoda H, Chan SL, Stern N, Palmer D, et al. Transarterial chemo-embolisation of hepatocellular carcinoma: impact of liver function and vascular invasion. Br. J. Cancer. 2017;116:448-454.

101. Lencioni R, de Baere T, Soulen MC, Rilling WS, Geschwind J-FH. Lipiodol transarterial chemoembolization for hepatocellular carcinoma: A systematic review of efficacy and safety data. Hepatology. 2016;64:106-116.

102. Meyer T, Kirkwood A, Roughton M, Beare S, Tsochatzis E, Yu D, et al. A randomised phase II/III trial of 3-weekly cisplatin-based sequential transarterial chemoembolisation vs embolisation alone for hepatocellular carcinoma. Br. J. Cancer. 2013;108:1252-1259.

103. Lammer J, Malagari K, Vogl T, Pilleul F, Denys A, Watkinson A, et al. Prospective randomized study of doxorubicin-eluting-bead embolization in the treatment of hepatocellular carcinoma: results of the PRECISION V study. Cardiovasc. Intervent. Radiol. 2010;33:41-52. 
104. de Baere T, Arai Y, Lencioni R, Geschwind J-F, Rilling W, Salem R, et al. Treatment of Liver Tumors with Lipiodol TACE: Technical Recommendations from Experts Opinion. Cardiovasc. Intervent. Radiol. 2016;39:334-343.

105. Gillmore R, Stuart S, Kirkwood A, Hameeduddin A, Woodward N, Burroughs AK, et al. EASL and mRECIST responses are independent prognostic factors for survival in hepatocellular cancer patients treated with transarterial embolization. J. Hepatol. 2011;55:1309-1316.

106. Vincenzi B, Di Maio M, Silletta M, D’Onofrio L, Spoto C, Piccirillo MC, et al. Prognostic Relevance of Objective Response According to EASL Criteria and mRECIST Criteria in Hepatocellular Carcinoma Patients Treated with Loco-Regional Therapies: A Literature-Based Meta-Analysis. PLoS One. 2015;10:e0133488.

107. Kudo M. Proposal of Primary Endpoints for TACE Combination Trials with Systemic Therapy: Lessons Learned from 5 Negative Trials and the Positive TACTICS Trial. Liver Cancer. 2018;7:225-234.

108. Kudo M, Ueshima K, Ikeda M, Torimura T, Tanabe N, Aikata H, et al. Randomised, multicentre prospective trial of transarterial chemoembolisation (TACE) plus sorafenib as compared with TACE alone in patients with hepatocellular carcinoma: TACTICS trial. Gut [Internet]. 2019;Available from: http://dx.doi.org/10.1136/gutjnl-2019-318934

109. Kudo M, Kubo S, Takayasu K, Sakamoto M, Tanaka M, Ikai I, et al. Response Evaluation Criteria in Cancer of the Liver (RECICL) proposed by the Liver Cancer Study Group of Japan (2009 Revised Version). Hepatol. Res. 2010;40:686-692.

110. Kudo M. A New Treatment Option for Intermediate-Stage Hepatocellular Carcinoma with High Tumor Burden: Initial Lenvatinib Therapy with Subsequent Selective TACE. Liver Cancer. 2019;8:299-311.

111. Therasse P, Arbuck SG, Eisenhauer EA, Wanders J, Kaplan RS, Rubinstein L, et al. New guidelines to evaluate the response to treatment in solid tumors. European Organization for Research and Treatment of Cancer, National Cancer Institute of the United States, National Cancer Institute of Canada. Journal of the National Cancer Institute. 2000;92:205-216.

112. Lencioni R. New data supporting modified RECIST (mRECIST) for Hepatocellular Carcinoma. Clin. Cancer Res. 2013;19:1312-1314.

113. Nishino M, Giobbie-Hurder A, Gargano M, Suda M, Ramaiya NH, Hodi FS. Developing a common language for tumor response to immunotherapy: immunerelated response criteria using unidimensional measurements. Clin. Cancer Res. 2013;19:3936-3943.

114. Bohnsack O, Hoos A, Ludajic K. Adaptation and modification of the immune related response criteria (IRRC): IrRECIST. J. Clin. Orthod. 2014;32:e22121-e22121.

115. Hodi FS, Ballinger M, Lyons B, Soria J-C, Nishino M, Tabernero J, et al. ImmuneModified Response Evaluation Criteria In Solid Tumors (imRECIST): Refining Guidelines to Assess the Clinical Benefit of Cancer Immunotherapy. J. Clin. Oncol. 2018;36:850-858.

116. Ikeda M, Sung MW, Kudo M, Kobayashi M, Baron AD, Finn RS, et al. A phase 1b 
trial of lenvatinib (LEN) plus pembrolizumab (PEM) in patients (pts) with unresectable hepatocellular carcinoma (uHCC). J. Clin. Oncol. 2018;36:4076-4076.

117. Moehler M, Heo J, Lee HC, Tak WY, Chao Y, Paik SW, et al. Vaccinia-based oncolytic immunotherapy Pexastimogene Devacirepvec in patients with advanced hepatocellular carcinoma after sorafenib failure: a randomized multicenter Phase Ilb trial (TRAVERSE). Oncoimmunology. 2019;8:1615817.

118. Heo J, Reid T, Ruo L, Breitbach CJ, Rose S, Bloomston M, et al. Randomized dose-finding clinical trial of oncolytic immunotherapeutic vaccinia JX-594 in liver cancer. Nat. Med. 2013;1-10.

119. Llovet JM, Zucman-Rossi J, Pikarsky E, Sangro B, Schwartz M, Sherman M, et al. Hepatocellular carcinoma. Nature Reviews Disease Primers. 2016;2:16018-16023.

120. Bruix J, Sherman M, American Association for the Study of Liver Diseases. Management of hepatocellular carcinoma: an update. Hepatology. 2011;53:10201022.

121. Qin S, Bai Y, Lim HY, Thongprasert S, Chao Y, Fan J, et al. Randomized, multicenter, open-label study of oxaliplatin plus fluorouracil/leucovorin versus doxorubicin as palliative chemotherapy in patients with advanced hepatocellular carcinoma from Asia. J. Clin. Oncol. 2013;31:3501-3508.

122. Abou-Alfa GK, Niedzwieski D, Knox JJ, Kaubisch A, Posey J, Tan BR, et al. Phase III randomized study of sorafenib plus doxorubicin versus sorafenib in patients with advanced hepatocellular carcinoma (HCC): CALGB 80802 (Alliance). J. Clin. Orthod. 2016;34:192-192.

123. Kudo M, Ueshima K, Yokosuka O, Ogasawara S, Obi S, Izumi N, et al. Sorafenib plus low-dose cisplatin and fluorouracil hepatic arterial infusion chemotherapy versus sorafenib alone in patients with advanced hepatocellular carcinoma (SILIUS): a randomised, open label, phase 3 trial. Lancet Gastroenterol Hepatol. 2018;3:424432.

124. Yeo W, Mok TS, Zee B, Leung TWT, Lai PBS, Lau WY, et al. A randomized phase III study of doxorubicin versus cisplatin/interferon alpha-2b/doxorubicin/fluorouracil (PIAF) combination chemotherapy for unresectable hepatocellular carcinoma. J. Natl. Cancer Inst. 2005;97:1532-1538.

125. Chow PK h., Tai B-C, Tan C-K, Machin D, Win KM, Johnson PJ, et al. High-dose tamoxifen in the treatment of inoperable hepatocellular carcinoma: A multicenter randomized controlled trial. Hepatology. 2002;36:1221-1226.

126. Johnson PJ, Qin S, Park J-W, Poon RTP, Raoul J-L, Philip PA, et al. Brivanib versus sorafenib as first-line therapy in patients with unresectable, advanced hepatocellular carcinoma: results from the randomized phase III BRISK-FL study. J. Clin. Oncol. 2013;31:3517-3524.

127. Llovet JM, Decaens T, Raoul JL, Boucher E, Kudo M, Chang C, et al. Brivanib in patients with advanced hepatocellular carcinoma who were intolerant to sorafenib or for whom sorafenib failed: results from the randomized phase III BRISK-PS study. J. Clin. Oncol. 2013;31:3509-3516. 
128. Cainap C, Qin S, Huang W-T, Chung IJ, Pan H, Cheng Y, et al. Linifanib versus Sorafenib in patients with advanced hepatocellular carcinoma: results of a randomized phase III trial. J. Clin. Oncol. 2015;33:172-179.

129. Zhu AX, Rosmorduc O, Evans TRJ, Ross PJ, Santoro A, Carrilho FJ, et al. SEARCH: a phase III, randomized, double-blind, placebo-controlled trial of sorafenib plus erlotinib in patients with advanced hepatocellular carcinoma. J. Clin. Oncol. 2015;33:559-566.

130. Zhu AX, Kudo M, Assenat E, Cattan S, Kang Y-K, Lim HY, et al. Effect of everolimus on survival in advanced hepatocellular carcinoma after failure of sorafenib: the EVOLVE-1 randomized clinical trial. JAMA. 2014;312:57-67.

131. Rimassa L, Assenat E, Peck-Radosavljevic M, Pracht M, Zagonel V, Mathurin P, et al. Tivantinib for second-line treatment of MET-high, advanced hepatocellular carcinoma (METIV-HCC): a final analysis of a phase 3, randomised, placebocontrolled study. Lancet Oncol. [Internet]. 2018 [cited 2018 Apr 4];Available from: http://dx.doi.org/10.1016/S1470-2045(18)30146-3

132. Merle P, Blanc J-F, Phelip J-M, Pelletier G, Bronowicki J-P, Touchefeu Y, et al. Doxorubicin-loaded nanoparticles for patients with advanced hepatocellular carcinoma after sorafenib treatment failure (RELIVE): a phase 3 randomised controlled trial. Lancet Gastroenterol Hepatol. 2019;4:454-465.

133. Abou-Alfa GK, Qin S, Ryoo B-Y, Lu S-N, Yen C-J, Feng Y-H, et al. Phase III randomized study of second line ADI-PEG 20 plus best supportive care versus placebo plus best supportive care in patients with advanced hepatocellular carcinoma. Ann. Oncol. 2018;29:1402-1408.

134. Roche's Tecentriq in combination with Avastin increased overall survival and progression-free survival in people with unresectable hepatocellular carcinoma [Internet]. [cited 2019 Nov 4];Available from:

https://www.roche.com/media/releases/med-cor-2019-10-21.htm

135. Zhu AX, Park JO, Ryoo B-Y, Yen C-J, Poon R, Pastorelli D, et al. Ramucirumab versus placebo as second-line treatment in patients with advanced hepatocellular carcinoma following first-line therapy with sorafenib (REACH): a randomised, doubleblind, multicentre, phase 3 trial. Lancet Oncol. 2015;16:859-870.

136. Llovet JM, Pena CEA, Lathia CD, Shan M, Meinhardt G, Bruix J, et al. Plasma Biomarkers as Predictors of Outcome in Patients with Advanced Hepatocellular Carcinoma. Clin. Cancer Res. 2012;18:2290-2300.

137. Vilgrain V, Pereira H, Assenat E, Guiu B, llonca AD, Pageaux G-P, et al. Efficacy and safety of selective internal radiotherapy with yttrium-90 resin microspheres compared with sorafenib in locally advanced and inoperable hepatocellular carcinoma (SARAH): an open-label randomised controlled phase 3 trial. Lancet Oncol. [Internet]. 2017;Available from: http://dx.doi.org/10.1016/S1470-2045(17)30683-6

138. Chow PKH, Gandhi M, Tan S-B, Khin MW, Khasbazar A, Ong J, et al. SIRveNIB: Selective Internal Radiation Therapy Versus Sorafenib in Asia-Pacific Patients With Hepatocellular Carcinoma. J. Clin. Oncol. 2018;36:1913-1921.

139. Ricke J, Sangro B, Amthauer H, Bargellini I, Bartenstein P, De Toni E, et al. The 
impact of combining Selective Internal Radiation Therapy (SIRT) with Sorafenib on overall survival in patients with advanced hepatocellular carcinoma: The Soramic trial palliative cohort. J. Hepatol. 2018;68:S102.

140. Cheng A-L, Kang Y-K, Chen Z, Tsao C-J, Qin S, Kim JS, et al. Efficacy and safety of sorafenib in patients in the Asia-Pacific region with advanced hepatocellular carcinoma: a phase III randomised, double-blind, placebo-controlled trial. Lancet Oncol. 2009;10:25-34.

141. He M, Li Q, Zou R, Shen J, Fang W, Tan G, et al. Sorafenib Plus Hepatic Arterial Infusion of Oxaliplatin, Fluorouracil, and Leucovorin vs Sorafenib Alone for Hepatocellular Carcinoma With Portal Vein Invasion: A Randomized Clinical Trial. JAMA Oncol [Internet]. 2019;Available from:

http://dx.doi.org/10.1001/jamaoncol.2019.0250

142. Jouve J-L, Lecomte T, Bouché O, Barbier E, Khemissa Akouz F, Riachi G, et al. Pravastatin combination with sorafenib does not improve survival in advanced hepatocellular carcinoma. J. Hepatol. 2019;71:516-522.

143. Abou-Alfa GK, Shi Q, Knox JJ, Kaubisch A, Niedzwiecki D, Posey J, et al. Assessment of Treatment With Sorafenib Plus Doxorubicin vs Sorafenib Alone in Patients With Advanced Hepatocellular Carcinoma: Phase 3 CALGB 80802 Randomized Clinical Trial. JAMA Oncol [Internet]. 2019;Available from: http://dx.doi.org/10.1001/jamaoncol.2019.2792

144. Torre LA, Bray F, Siegel RL, Ferlay J, Lortet-Tieulent J, Jemal A. Global cancer statistics, 2012. CA Cancer J. Clin. 2015;65:87-108.

145. Gan HK, You B, Pond GR, Chen EX. Assumptions of expected benefits in randomized phase III trials evaluating systemic treatments for cancer. J. Natl. Cancer Inst. 2012;104:590-598.

146. Oxnard GR, Wilcox KH, Gonen M, Polotsky M, Hirsch BR, Schwartz LH. Response Rate as a Regulatory End Point in Single-Arm Studies of Advanced Solid Tumors. JAMA Oncol. 2016;2:772-779.

147. Sangro B, Gomez-Martin C, de la Mata M, Iñarrairaegui M, Garralda E, Barrera P, et al. A clinical trial of CTLA-4 blockade with tremelimumab in patients with hepatocellular carcinoma and chronic hepatitis C. J. Hepatol. 2013;59:81-88.

148. Qin SK, Ren ZG, Meng ZQ, Chen ZD, Chai XL, Xiong JP, et al. LBA27A randomized multicentered phase II study to evaluate SHR-1210 (PD-1 antibody) in subjects with advanced hepatocellular carcinoma (HCC) who failed or intolerable to prior systemic treatment. Ann. Oncol. [Internet]. 2018 [cited 2019 Sep 23];29. Available from: https://academic.oup.com/annonc/article/29/suppl_8/mdy424.029/5141688

149. Finn RS, Ryoo B-Y, Merle P, Kudo M, Bouattour M, Lim H-Y, et al. Results of KEYNOTE-240: phase 3 study of pembrolizumab (Pembro) vs best supportive care (BSC) for second line therapy in advanced hepatocellular carcinoma (HCC). J. Clin. Orthod. 2019;37:4004-4004.

150. Duffy AG, Ulahannan SV, Makorova-Rusher O, Rahma O, Wedemeyer H, Pratt D, et al. Tremelimumab in combination with ablation in patients with advanced 
hepatocellular carcinoma. J. Hepatol. 2017;66:545-551.

151. Yau T, Kang Y-K, Kim T-Y, El-Khoueiry AB, Santoro A, Sangro B, et al. Nivolumab (NIVO) + ipilimumab (IPI) combination therapy in patients (pts) with advanced hepatocellular carcinoma (aHCC): Results from CheckMate 040. J. Clin. Orthod. 2019;37:4012-4012.

152. Greten TF, Lai CW, Li G, Staveley-O'Carroll KF. Targeted and Immune-Based Therapies for Hepatocellular Carcinoma. Gastroenterology. 2019;156:510-524.

153. Greten TF, Sangro B. Targets for immunotherapy of liver cancer. J. Hepatol. [Internet]. 2017;Available from: http://dx.doi.org/10.1016/j.jhep.2017.09.007

154. Anagnostou V, Yarchoan M, Hansen AR, Wang H, Verde F, Sharon E, et al. Immuno-oncology Trial Endpoints: Capturing Clinically Meaningful Activity. Clin. Cancer Res. 2017;23:4959-4969.

155. Ritchie G, Gasper H, Man J, Lord S, Marschner I, Friedlander M, et al. Defining the Most Appropriate Primary End Point in Phase 2 Trials of Immune Checkpoint Inhibitors for Advanced Solid Cancers: A Systematic Review and Meta-analysis. JAMA Oncol. 2018;4:522-528.

156. Simon RM, Paik S, Hayes DF. Use of Archived Specimens in Evaluation of Prognostic and Predictive Biomarkers. JNCl Journal of the National Cancer Institute. 2009;101:1446-1452.

157. Mandrekar SJ, Sargent DJ. Clinical trial designs for predictive biomarker validation: theoretical considerations and practical challenges. J. Clin. Oncol. 2009;27:40274034.

158. Mandrekar SJ, Sargent DJ. Genomic advances and their impact on clinical trial design. Genome Med. 2009;1:69.

159. Renfro LA, An M-W, Mandrekar SJ. Precision oncology: A new era of cancer clinical trials. Cancer Lett. 2017;387:121-126.

160. Biomarker defintion $\mathrm{NCl}$ [Internet]. Available from: https://www.cancer.gov/publications/dictionaries/cancer-terms/def/biomarker

161. Rebouissou S, La Bella T, Rekik S, Imbeaud S, Calatayud A-L, Rohr-Udilova N, et al. Proliferation Markers Are Associated with MET Expression in Hepatocellular Carcinoma and Predict Tivantinib Sensitivity In Vitro. Clin. Cancer Res. [Internet]. 2017;Available from: http://dx.doi.org/10.1158/1078-0432.CCR-16-3118

162. Zhu AX, Baron AD, Malfertheiner P, Kudo M, Kawazoe S, Pezet D, et al. Ramucirumab as Second-Line Treatment in Patients With Advanced Hepatocellular Carcinoma: Analysis of REACH Trial Results by Child-Pugh Score. JAMA Oncol. 2017;3:235-243.

163. Kim RD, Sarker D, Meyer T, Yau T, Macarulla T, Park J-W, et al. First-in-Human Phase I Study of Fisogatinib (BLU-554) Validates Aberrant Fibroblast Growth Factor 19 Signaling as a Driver Event in Hepatocellular Carcinoma. Cancer Discov. [Internet]. 2019;Available from: http://dx.doi.org/10.1158/2159-8290.CD-19-0555 
164. Sia D, Jiao Y, Martinez-Quetglas I, Kuchuk O, Villacorta-Martin C, Castro de Moura $\mathrm{M}$, et al. Identification of an Immune-specific Class of Hepatocellular Carcinoma, Based on Molecular Features. Gastroenterology. 2017;153:812-826.

165. Finn RS, Kudo M, Cheng A-L, Wyrwicz L, Ngan R, Blanc J-F, et al. LBA30Analysis of serum biomarkers (BM) in patients (pts) from a phase 3 study of lenvatinib (LEN) vs sorafenib (SOR) as first-line treatment for unresectable hepatocellular carcinoma (uHCC). Ann. Oncol. [Internet]. 2017 [cited 2019 Jul 26];28. Available from: https://academic.oup.com/annonc/article-abstract/28/suppl_5/mdx440.022/4109934

166. Kaseb AO, Carmagnani Pestana R, Vence LM, Blando JM, Singh S, Ikoma N, et al. Randomized, open-label, perioperative phase II study evaluating nivolumab alone versus nivolumab plus ipilimumab in patients with resectable HCC. J. Clin. Orthod. 2019;37:185-185.

167. Kwak EL, Bang Y-J, Camidge DR, Shaw AT, Solomon B, Maki RG, et al. Anaplastic lymphoma kinase inhibition in non-small-cell lung cancer. N. Engl. J. Med. 2010;363:1693-1703.

168. Lynch TJ, Bell DW, Sordella R, Gurubhagavatula S, Okimoto RA, Brannigan BW, et al. Activating mutations in the epidermal growth factor receptor underlying responsiveness of non-small-cell lung cancer to gefitinib. N. Engl. J. Med. 2004;350:2129-2139.

169. Slamon DJ, Leyland-Jones B, Shak S, Fuchs H, Paton V, Bajamonde A, et al. Use of chemotherapy plus a monoclonal antibody against HER2 for metastatic breast cancer that overexpresses HER2. N. Engl. J. Med. 2001;344:783-792.

170. Finn RS, Martin M, Rugo HS, Jones S, Im S-A, Gelmon K, et al. Palbociclib and Letrozole in Advanced Breast Cancer. N. Engl. J. Med. 2016;375:1925-1936.

171. Demetri GD, von Mehren M, Blanke CD, Van den Abbeele AD, Eisenberg B, Roberts PJ, et al. Efficacy and safety of imatinib mesylate in advanced gastrointestinal stromal tumors. N. Engl. J. Med. 2002;347:472-480.

172. Druker BJ, Guilhot F, O'Brien SG, Gathmann I, Kantarjian H, Gattermann N, et al. Five-year follow-up of patients receiving imatinib for chronic myeloid leukemia. N. Engl. J. Med. 2006;355:2408-2417.

173. Pantel K. Blood-Based Analysis of Circulating Cell-Free DNA and Tumor Cells for Early Cancer Detection. PLoS Med. 2016;13:e1002205.

174. Mandrekar SJ, An M-W, Sargent DJ. A review of phase II trial designs for initial marker validation. Contemp. Clin. Trials. 2013;36:597-604.

175. Labgaa I, Villanueva A. Liquid biopsy in liver cancer. Discov. Med. 2015;19:263273.

176. Corcoran RB, Chabner BA. Application of Cell-free DNA Analysis to Cancer Treatment. N. Engl. J. Med. 2018;379:1754-1765.

177. Wan JCM, Massie C, Garcia-Corbacho J, Mouliere F, Brenton JD, Caldas C, et al. Liquid biopsies come of age: towards implementation of circulating tumour DNA. Nat. Rev. Cancer. 2017;17:223-238. 
178. Office of the Commissioner. FDA approves first blood test to detect gene mutation associated with non-small cell lung cancer [Internet]. 2016 [cited 2019 May 16];Available from: https://www.fda.gov/news-events/press-announcements/fdaapproves-first-blood-test-detect-gene-mutation-associated-non-small-cell-lung-cancer

179. Labgaa I, Villacorta-Martin C, D'Avola D, Craig AJ, von Felden J, Martins-Filho SN, et al. A pilot study of ultra-deep targeted sequencing of plasma DNA identifies driver mutations in hepatocellular carcinoma. Oncogene. 2018;37:3740-3752.

180. Qu C, Wang Y, Wang P, Chen K, Wang M, Zeng H, et al. Detection of early-stage hepatocellular carcinoma in asymptomatic HBsAg-seropositive individuals by liquid biopsy. Proc. Natl. Acad. Sci. U. S. A. [Internet]. 2019;Available from: http://dx.doi.org/10.1073/pnas.1819799116

181. Xu R-H, Wei W, Krawczyk M, Wang W, Luo H, Flagg K, et al. Circulating tumour DNA methylation markers for diagnosis and prognosis of hepatocellular carcinoma. Nat. Mater. 2017;16:1155-1161.

182. Bhan I, Mosesso K, Goyal L, Philipp J, Kalinich M, Franses JW, et al. Detection and Analysis of Circulating Epithelial Cells in Liquid Biopsies From Patients With Liver Disease. Gastroenterology [Internet]. 2018;Available from: http://dx.doi.org/10.1053/j.gastro.2018.09.020

183. Oh CR, Kong S-Y, Im H-S, Kim HJ, Kim MK, Yoon K-A, et al. Genome-wide copy number alteration and VEGFA amplification of circulating cell-free DNA as a biomarker in advanced hepatocellular carcinoma patients treated with Sorafenib. BMC Cancer. 2019;19:292.

184. Lim HY, Merle P, Weiss KH, Yau TC, Ross P, Mazzaferro V, et al. Phase II Studies with Refametinib or Refametinib plus Sorafenib in Patients with RAS-mutated Hepatocellular Carcinoma. Clin. Cancer Res. [Internet]. 2018;Available from: http://dx.doi.org/10.1158/1078-0432.CCR-17-3588

185. Chie W-C, Blazeby JM, Hsiao C-F, Chiu H-C, Poon RT, Mikoshiba N, et al. International cross-cultural field validation of an European Organization for Research and Treatment of Cancer questionnaire module for patients with primary liver cancer, the European Organization for Research and Treatment of Cancer quality-of-life questionnaire HCC18. Hepatology. 2012;55:1122-1129.

186. Blazeby JM, Currie E, Zee BCY, Chie W-C, Poon RT, Garden OJ, et al. Development of a questionnaire module to supplement the EORTC QLQ-C30 to assess quality of life in patients with hepatocellular carcinoma, the EORTC QLQHCC18. Eur. J. Cancer. 2004;40:2439-2444.

187. Huang G, Chen X, Lau WY, Shen F, Wang RY, Yuan SX, et al. Quality of life after surgical resection compared with radiofrequency ablation for small hepatocellular carcinomas. Br. J. Surg. 2014;101:1006-1015.

188. Salem R, Gilbertsen M, Butt Z, Memon K, Vouche M, Hickey R, et al. Increased quality of life among hepatocellular carcinoma patients treated with radioembolization, compared with chemoembolization. Clin. Gastroenterol. Hepatol. 2013;11:13581365.e1.

189. Kudo M, Trevisani F, Abou-Alfa GK, Rimassa L. Hepatocellular Carcinoma: 
Therapeutic Guidelines and Medical Treatment. Liver Cancer. 2016;6:16-26.

190. Omata M, Cheng A-L, Kokudo N, Kudo M, Lee JM, Jia J, et al. Asia-Pacific clinical practice guidelines on the management of hepatocellular carcinoma: a 2017 update. Hepatol. Int. 2017;11:317-370.

191. Kokudo N, Takemura N, Hasegawa K, Takayama T, Kubo S, Shimada M, et al. Clinical practice guidelines for hepatocellular carcinoma: The Japan Society of Hepatology 2017 (4th JSH-HCC guidelines) 2019 update. Hepatol. Res. [Internet]. 2019;Available from: http://dx.doi.org/10.1111/hepr.13411

192. Surveillance group, Diagnosis group, Staging group, Surgery group, Local ablation group, TACE/TARE/HAI group, et al. Management consensus guideline for hepatocellular carcinoma: 2016 updated by the Taiwan Liver Cancer Association and the Gastroenterological Society of Taiwan. J. Formos. Med. Assoc. 2018;117:381403.

193. Zhou J, Sun H-C, Wang Z, Cong W-M, Wang J-H, Zeng M-S, et al. Guidelines for Diagnosis and Treatment of Primary Liver Cancer in China (2017 Edition). Liver Cancer. 2018;7:235-260.

194. Korean Liver Cancer Association, National Cancer Center. 2018 Korean Liver Cancer Association-National Cancer Center Korea Practice Guidelines for the Management of Hepatocellular Carcinoma. Gut Liver. 2019;13:227-299.

195. Sharma $P$, Allison JP. The future of immune checkpoint therapy. Science. 2015;348:56-61.

196. Fukumura D, Kloepper J, Amoozgar Z, Duda DG, Jain RK. Enhancing cancer immunotherapy using antiangiogenics: opportunities and challenges. Nat. Rev. Clin. Oncol. 2018;15:325-340.

197. Kalbasi A, Ribas A. Tumour-intrinsic resistance to immune checkpoint blockade. Nat. Rev. Immunol. 2020;20:25-39.

198. Ruiz de Galarreta M, Bresnahan E, Molina-Sanchez P, Lindblad KE, Maier B, Sia $D$, et al. $\beta$-catenin activation promotes immune escape and resistance to anti-PD-1 therapy in hepatocellular carcinoma. Cancer Discov. [Internet]. 2019;Available from: http://dx.doi.org/10.1158/2159-8290.CD-19-0074

199. Pinyol R, Sia D, Llovet JM. Immune exclusion-Wnt/CTNNB1 class predicts resistance to immunotherapies in HCC. Clin. Cancer Res. [Internet]. 2019;Available from: http://dx.doi.org/10.1158/1078-0432.CCR-18-3778

200. Harding JJ, Nandakumar S, Armenia J, Khalil DN, Albano M, Ly M, et al. Prospective Genotyping of Hepatocellular Carcinoma: Clinical Implications of Next Generation Sequencing for Matching Patients to Targeted and Immune Therapies. Clin. Cancer Res. [Internet]. 2018;Available from: http://dx.doi.org/10.1158/10780432.CCR-18-2293 
Figure 1

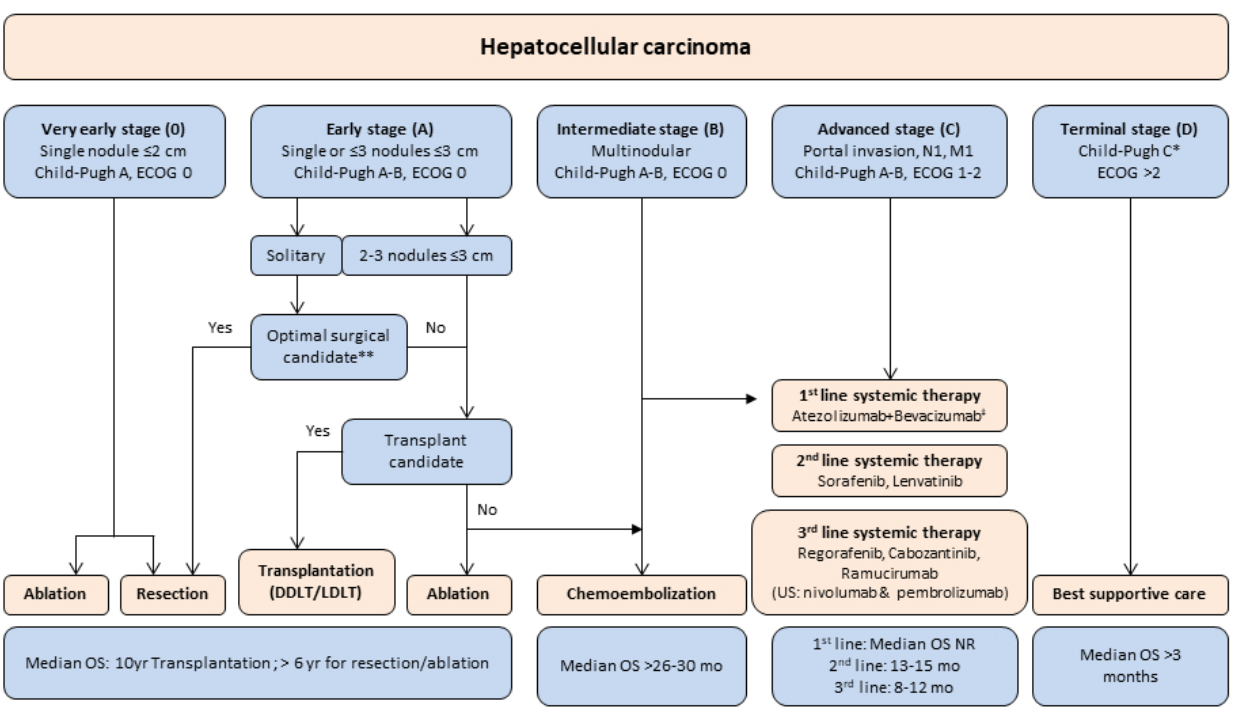

Figure 1

$254 \times 190 \mathrm{~mm}(96 \times 96 \mathrm{DPI})$

Hepatology 
Figure 2A

First line

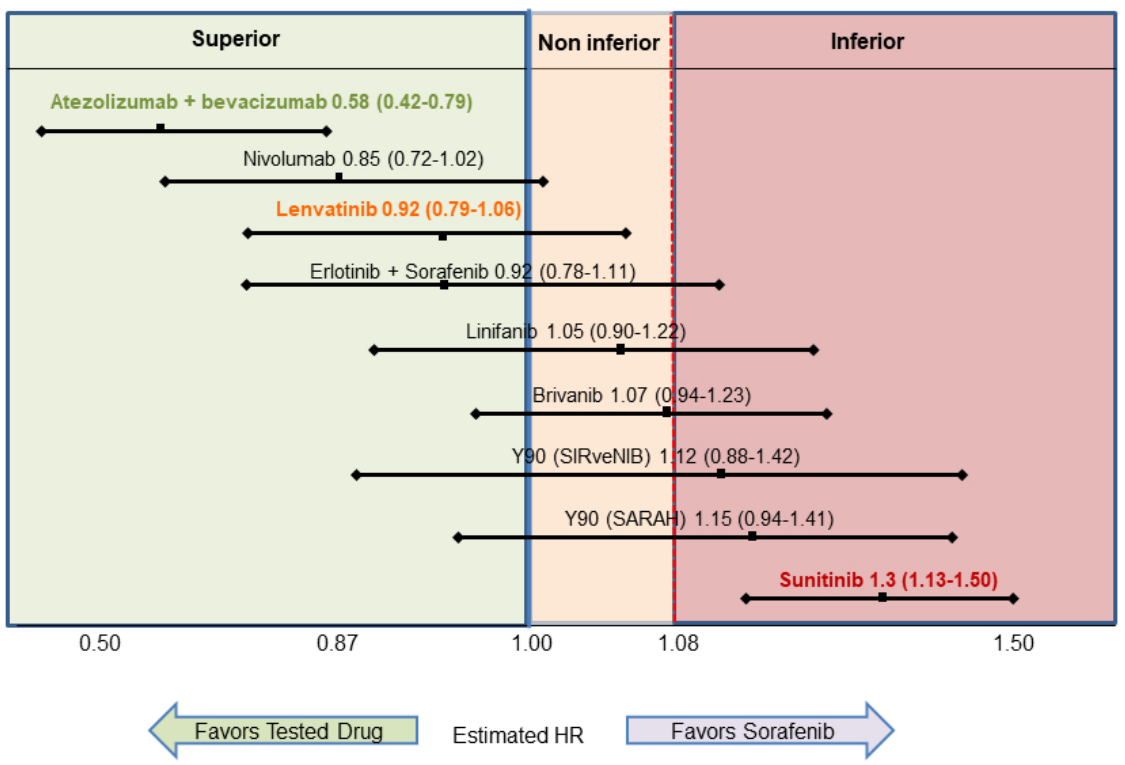

Figure 2A

$254 \times 190 \mathrm{~mm}(96 \times 96$ DPI)

Hepatology 
1

2

3

4

5

6

7

8

9

10

11

12

13

14

15

16

17

18

19

20

21

22

23

24

25

26

27

28

29

30

31

32

33

34

35

36

37

38

39

40

41

42

43

44

45

46

47

48

49

50

51

52

53

54

55

56

57

58

59

60

Figure 2B

Secondline

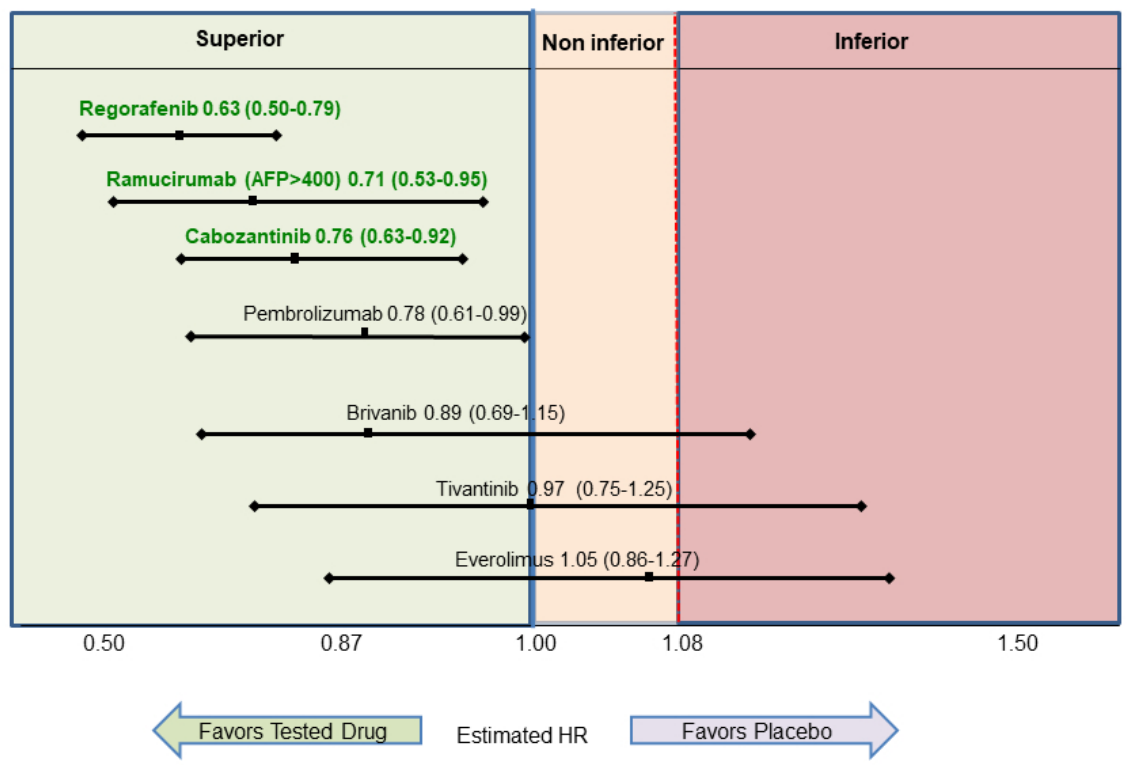

Figure 2B

254x190mm (96 x 96 DPI)

Hepatology 
Figure 3

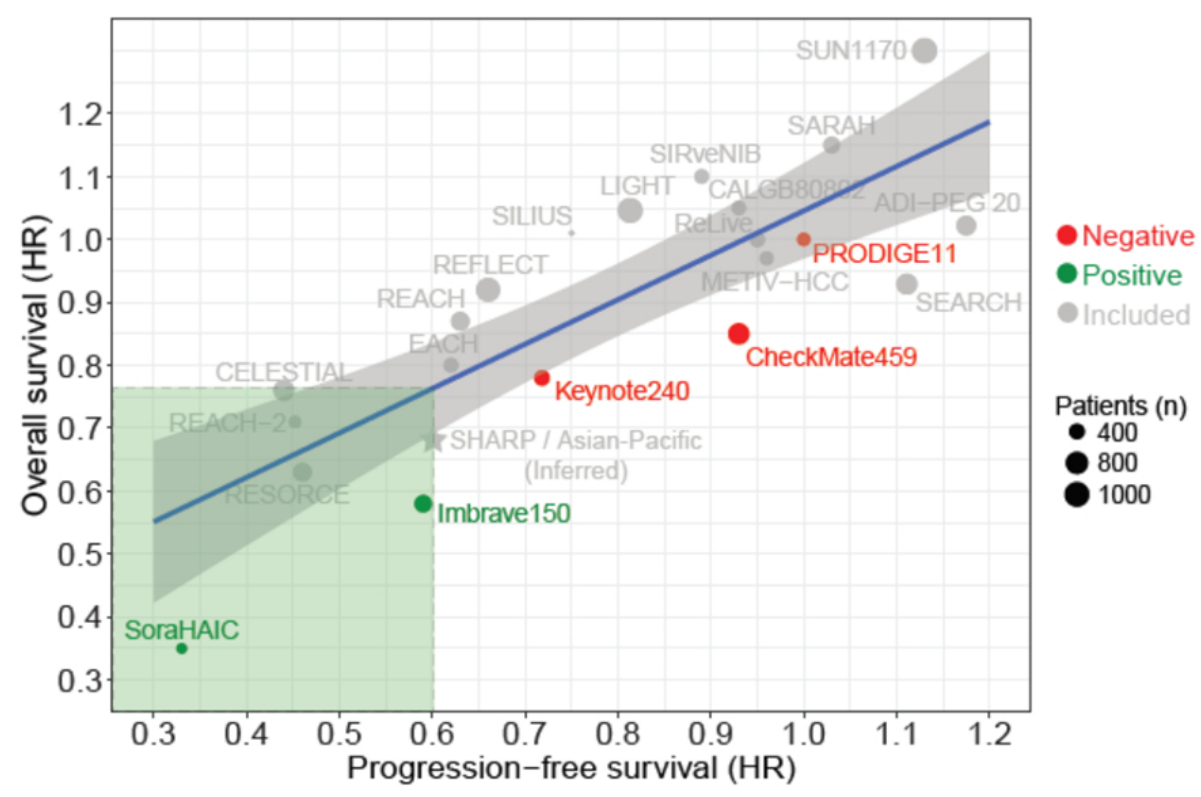

Figure 3

$254 \times 190 m m(96 \times 96$ DPI)

Hepatology 